\title{
LIOUVILLE FIRST-PASSAGE PERCOLATION: SUBSEQUENTIAL SCALING LIMITS AT HIGH TEMPERATURE
}

\author{
By JiAn DING ${ }^{1}$ AND ALEXANDER DUNLAP ${ }^{2}$ \\ University of Pennsylvania and Stanford University
}

Let $\left\{Y_{\mathfrak{B}}(x): x \in \mathfrak{B}\right\}$ be a discrete Gaussian free field in a twodimensional box $\mathfrak{B}$ of side length $S$ with Dirichlet boundary conditions. We study Liouville first-passage percolation: the shortest-path metric in which each vertex $x$ is given a weight of $e^{\gamma Y_{\mathfrak{B}}(x)}$ for some $\gamma>0$. We show that for sufficiently small but fixed $\gamma>0$, for any sequence of scales $\left\{S_{k}\right\}$ there exists a subsequence along which the appropriately scaled and interpolated Liouville FPP metric converges in the Gromov-Hausdorff sense to a random metric on the unit square in $\mathbf{R}^{2}$. In addition, all possible (conjecturally unique) scaling limits are homeomorphic by bi-Hölder-continuous homeomorphisms to the unit square with the Euclidean metric.

\section{CONTENTS}

1. Introduction . . . . . . . . . . . . . . . . . . . . . . 691

1.1. Background and related results . . . . . . . . . . . . . . . . . . 692

1.2. Proof approach and the RSW method . . . . . . . . . . . . . . . . . . . 693

2. Preliminaries . . . . . . . . . . . . . . . . . . . . . . . . 694

2.1. Notational conventions . . . . . . . . . . . . . . . . . . . . . . . . . . 694

2.1.1. Boxes . . . . . . . . . . . . . . . . . . . . . . . . . 6 694

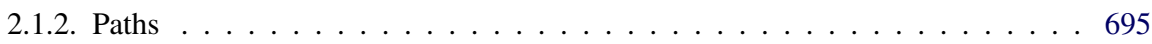

2.1.3. Asymptotics . . . . . . . . . . . . . . . . . . . . . . . . . . . . . . . . 696

2.2. Properties of the field . . . . . . . . . . . . . . . . . . . . . . . 696

2.2.1. Coupling of fields in different boxes . . . . . . . . . . . . . . . . . . . . . . . . 696 697

2.2.2. Description of the criteria for the field . . . . . . . . . . . . . . . . . 697

2.2.3. Proof that the DGFF satisfies the criteria . . . . . . . . . . . . . . 698

2.2.4. Further properties of the field . . . . . . . . . . . . . . . 700

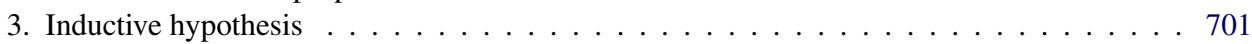

4. Crossing quantile lower bounds … . . . . . . . . . . . . . . 702

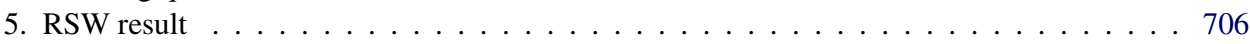

5.1. Scale and aspect ratio setup . . . . . . . . . . . . . . . . . 706

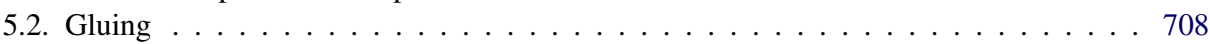

5.3. Multiscale analysis . . . . . . . . . . . . . . . . . . . . . 717

6. Upper bounds on FPP distance and geodesic length . . . . . . . . . . . . . . . . 724

Received September 2016; revised November 2017.

${ }^{1}$ Supported in part by NSF Grant DMS-1455049 and an Alfred Sloan fellowship.

${ }^{2}$ Supported in part by an NSF Graduate Research Fellowship.

MSC2010 subject classifications. Primary 60K35; secondary 60G60, 60B43.

Key words and phrases. Liouville first-passage percolation, discrete Gaussian free field, RussoSeymour-Welsh method, Liouville quantum gravity. 
6.1. Crossing distance upper bound . . . . . . . . . . . . . . . . . . . . . . . 724

6.2. Expected geodesic length upper bound . . . . . . . . . . . . . . . . . . 726

6.3. Diameter upper bound . . . . . . . . . . . . . . . . . . . . . . 727

7. Variation upper bounds . . . . . . . . . . . . . . . . . . . . . 730

7.1. Variance of the crossing distance . . . . . . . . . . . . . . . . . 730

7.2. Coefficient of variation . . . . . . . . . . . . . . . . . . . . . . . 734

8. Subsequential limits of FPP metrics . . . . . . . . . . . . . . . . . . . 735

8.1. Tightness and subsequential convergence . . . . . . . . . . . . . . 736

8.2. Hölder-continuity of limiting metrics . . . . . . . . . . . . . . . . . . . . . . . . . . . . . . . . . . . . . . . . .

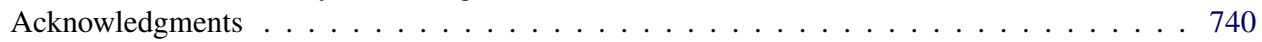

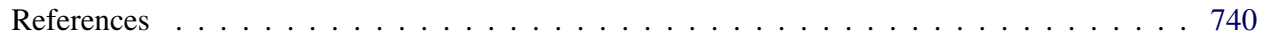

1. Introduction. We consider Liouville first-passage percolation, that is, firstpassage percolation on the exponential of the discrete Gaussian free field. Given a box (by which we mean a discrete rectangle) $\mathfrak{B} \subset \mathbf{Z}^{2}$, define $\overline{\mathfrak{B}}$, the blow-up of $\mathfrak{B}$, as the box three times larger in each dimension centered around $\mathfrak{B}$, and define $\partial \overline{\mathfrak{B}}$ to be the set of points whose Euclidean distance from $\overline{\mathfrak{B}}$ is exactly 1 . What we will call the discrete Gaussian free field on $\mathfrak{B}$ is the restriction to $\mathfrak{B}$ of the standard discrete Gaussian free field with Dirichlet boundary conditions on $\overline{\mathfrak{B}}$. This is the mean-zero Gaussian process $Y_{\mathfrak{B}}(x)$ such that $Y_{\mathfrak{B}}(x)=0$ for all $x \in \partial \overline{\mathfrak{B}}$ and $\mathbf{E} Y_{\mathfrak{B}}(x) Y_{\mathfrak{B}}(y)=G_{\overline{\mathfrak{B}}}(x, y)$ for all $x, y \in \overline{\mathfrak{B}}$, where $G_{\overline{\mathfrak{B}}}(x, y)$ is the Green's function of simple random walk on $\overline{\mathfrak{B}}$. (The constant 3 in the definition of the blow-up is irrelevant to the result-the point is that Dirichlet boundary conditions are imposed on a box which is a constant fraction larger.)

Fix an inverse-temperature parameter $\gamma>0$. Let $\mathfrak{B}_{S}=[0, S)^{2} \cap \mathbf{Z}^{2}$. We define the Liouville first-passage percolation metric dist ${ }_{S}$ on $\mathfrak{B}_{S}$ by

$$
\operatorname{dist}_{S}\left(x_{1}, x_{2}\right)=\min _{\pi} \sum_{x \in \pi} e^{\gamma Y_{\mathfrak{B}_{S}}(x)},
$$

where $\pi$ ranges over all paths in $\mathfrak{B}_{S}$ connecting $x_{1}$ and $x_{2}$. Given a sequence of normalizing constants $\kappa_{S}$, we define a metric $\widetilde{\mathrm{dist}_{S}}$ on $[0,1]^{2} \subset \mathbf{R}^{2}$ by letting

$$
\widetilde{\operatorname{dist}}_{S}\left(x_{1}, x_{2}\right)=\frac{1}{\kappa_{S}} \operatorname{dist}_{S}\left(S x_{1}, S x_{2}\right)
$$

for each $x_{1}, x_{2} \in[0,1]^{2} \cap \frac{1}{S} \mathbf{Z}^{2}$ and extending to all $x_{1}, x_{2} \in[0,1]^{2}$ by linear interpolation. We will prove the following.

THEOREM 1.1. There is a $\gamma_{0}>0$ so that, if $\gamma<\gamma_{0}$, then there exists a sequence of normalizing constants $\kappa_{S}$ so that, for every sequence of scales $S_{i}$, there is a subsequence $\left\{S_{i_{j}}\right\}$ so that $\widetilde{\text { dist }_{S_{i_{j}}}}$ converges in distribution (using the GromovHausdorff topology on the space of metrics) to a limiting metric, which moreover is homeomorphic to the Euclidean metric by a Hölder-continuous homeomorphism with Hölder-continuous inverse. 
REMARK 1.2. The $\gamma_{0}$ that we are able to establish is so small that calculating a precise value would be unilluminating. Extending our result to a "reasonable" value of $\gamma_{0}$ is an interesting open problem.

1.1. Background and related results. Substantial effort to date (see $[3,20]$ and their references) has been devoted to understanding classical first-passage percolation, with independent and identically distributed edge or vertex weights. We argue that FPP with strongly correlated weights is also a rich and interesting subject, involving questions both analogous to and distinctive from those asked in the classical case. In particular, since the Gaussian free field is in some sense the canonical strongly correlated random medium, we endeavor to study Liouville FPP - that is, FPP in $\mathbf{Z}^{2}$ with weights given by the exponential of DGFF.

More specifically, Liouville FPP is thought to play a key role in understanding the random metric associated with the Liouville quantum gravity (LQG) $[18,36$, 37]. It is a major open problem just to give a rigorous definition of such a metric. Miller and Sheffield have recently succeeded in giving such a definition for the case $\gamma=\sqrt{8 / 3}$; see $[17,31-34]$ and their references. In these papers, the authors focused on directly constructing the random metric in the continuum setup. Other recent work has shown the existence of scaling exponents for an attempt to construct LQG for $\gamma \in(0,2)$ via "LQG structure graphs" [21].

We take an alternative approach which seeks to understand the random metric of LQG via scaling limits of lattice approximations using the DGFF, as proposed (and discussed in more detail) in [5]. We choose to work with the square lattice-based Liouville FPP both for its simple formulation and for its relationship to classical FPP. Eventually, one might wish to tweak the definition of the discrete metric in order to obtain a scaling limit with more invariance properties. However, the methods developed in this article are robust to reasonable changes in the method of discretization. We make this precise by stating the necessary conditions on the field in Section 2.2.

Our result is similar in flavor to [23] and [26] which proved, respectively, that the graph distance of random quadrangulations has a subsequential scaling limit and that the all possible limiting metrics are homeomorphic to a 2-sphere. (In our case, however, the homeomorphism property is a byproduct of the compactness result.) The uniqueness of the scaling limit, known as the Brownian map, was proved in later works [24, 25, 29].

A crucial ingredient in [23] is a bijection [7, 9, 40] between uniform quadrangulations and labeled trees. In particular, such a bijection allows an explicit evaluation of the order of the typical distance in the random quadrangulation. By contrast, in our model, determining the FPP distance exponent seems to be a major challenge. Indeed, recent works $[10,11]$ have shown that the distance exponent for Liouville FPP is strictly less than 1 at high temperatures, and also [12] that there exists a family of log-correlated Gaussian fields for which the weight exponent can be arbitrarily close to 1 . This means that the distance exponent is not universal among 
log-correlated Gaussian fields, so precisely computing this exponent must involve rather subtle properties of the field. Our proof circumvents this difficulty since it works without knowing the scaling exponent.

1.2. Proof approach and the RSW method. The framework of our proof (which we note bears little similarity to the methods used in $[10,11]$ ) is a multiscale analysis procedure relying on several relationships which we establish between FPP distances at different scales. The key estimates are inductive upper and lower bounds on crossing distances and geodesic lengths, in which distances and lengths at a larger scale are estimated in terms of distances at a smaller scale. Most of the lower bounds on the larger-scale distances are achieved in Section 4 using percolation-type arguments, while the upper bounds on larger-scale distances and lengths are carried out in Section 6 using gluing arguments along with the lower bounds. In Section 6.3, we use a chaining argument to get an upper bound on box diameter, which combined with the lower bounds allows us to inductively bound the crossing distance coefficient of variation in Section 7. Finally, in Section 8, we apply this coefficient of variation bound to establish tightness, and thus subsequential convergence, of the normalized FPP metrics.

Carrying out the above strategy leads to a central problem: lower bounds on crossing distances are obtained in terms of "easy crossings" (between the two longer sides) of rectangles, while upper bounds are obtained in terms of "hard crossings" (between the two shorter sides) (see Figure 1). In order to play these bounds off of each other, we must establish a relationship between easy and hard crossing distances. Results of this type are known as RSW statements, and the key ingredient in our results (Section 5, representing the bulk of the paper) is an RSW theorem for the Liouville FPP setting.

We briefly review the history of the RSW method, an important technique in planar statistical physics, which was initiated in $[38,39,41]$ in order to prove a positive hard crossing probability through a rectangle in critical Bernoulli percolation. Recently, an RSW theory has been developed for FK percolation; see, for example, $[4,13,16]$. Most relevant to the present paper, an RSW theory was developed in [43] for Voronoi percolation. In fact, the beautiful method in [43] is widely applicable to percolation problems satisfying the FKG inequality, mild

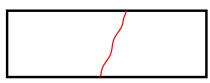

(a) Easy crossing

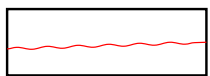

(b) Hard crossing

FIG. 1. Easy and hard crossings. 
symmetry assumptions and weak correlation between well-separated regions. For example, in [15], this method was used to give a simpler proof of the result of [4], and in [14], the authors proved an RSW theorem for the crossing probability of level sets of a planar Gaussian free field. The Liouville FPP model has analogous symmetry and correlation properties, indicating that the methods in [43] can apply in this setting as well. Indeed, the geometric framework of our RSW proof is hugely inspired by [43]. Shortly after we posted this article, in [2] the authors developed a method of comparing easy and hard crossing probabilities in the study of the Poisson-Boolean percolation model, although their method does not seem to apply to the geodesic of our FPP metric.

A main novelty of our result is that it seems to be the first RSW theorem for random planar metrics (rather than for traditional crossing probabilities for percolation problems). The use of RSW theory in the metric setting has the potential to enrich both the application and the theory of the RSW method, and we expect more applications of RSW theory in the study of random planar metrics. One encounters substantial challenges working with the FPP weights in our RSW result even given the beautiful work of [43]: the proof method of [43] is based on an intricate induction which becomes even more delicate with the FPP weights taken into account. Besides that, our FPP metric lacks a natural self-duality, which precludes using the hypothesis of crossing square boxes as in the traditional setup; rather, we start with "easy" crossings of rectangular boxes. The difficulties are such that we are only able to relate different quantiles of the FPP distance in different scales, and we have to apply our induction hypothesis on the variance of the FPP distance to relate different quantiles at each scale. This introduces an additional layer of complexity to our arguments.

\section{Preliminaries.}

2.1. Notational conventions. Here we introduce notation that we will use throughout the paper.

2.1.1. Boxes. Since we will be primarily working in the discrete setting, throughout the paper, the notation $[a, b)$ will denote the set of integers between $a$ and $b-1$, inclusive, and $[a, b]$ the set of integers between $a$ and $b$, inclusive. When we need to refer to an interval of real numbers, we will attach a subscript $\mathbf{R}$, as in $[a, b]_{\mathbf{R}}$, etc. A box or rectangle (we use the terms interchangeably) is a finite rectangular subset of $\mathbf{Z}^{2}$. We will denote by $\mathscr{B}$ the set of all boxes in $\mathbf{Z}^{d}$. We will say that a square box is dyadic if its side-length is a power of 2 and the coordinates of its bottom-left corner are multiples of its side-length. As in the Introduction, the blow-up of a box $\mathfrak{B}$, denoted $\overline{\mathfrak{B}}$, is the union of the nine translates of $\mathfrak{B}$ centered around $\mathfrak{B}$. We say that a rectangular box is portrait if its height is greater than its width and landscape if its width is greater than its height. For boxes $\mathfrak{A} \subseteq \mathfrak{B}$, we will use the notation $|\mathfrak{B} / \mathfrak{A}|$ to denote the maximum of the width of $\mathfrak{B}$ divided by the width of $\mathfrak{A}$ and the height of $\mathfrak{B}$ divided by the height of $\mathfrak{A}$. 
2.1.2. Paths. Suppose $\pi$ is a path and $Y$ is a random field. Define

$$
\psi(\pi ; Y)=\sum_{x \in \pi} \exp (\gamma Y(x)) .
$$

If $\mathfrak{R}$ is a rectangle, let

$$
\Psi_{\mathrm{LR}}(\Re ; Y)=\min _{\pi} \psi(\pi ; Y),
$$

where $\pi$ ranges over all left-right crossings of $\Re$. Define $\Psi_{\mathrm{BT}}$ analogously for bottom-top crossings. Also put

$$
\Psi_{\text {easy }}(\Re ; Y)=\min _{\pi} \psi(\pi ; Y),
$$

where $\pi$ ranges over all crossings between the longer sides of $\mathfrak{R}$, and let

$$
\Psi_{\text {hard }}(\Re ; Y)=\min _{\pi} \psi(\pi ; Y),
$$

where $\pi$ ranges over all crossings between the shorter sides of $\mathfrak{R}$. (Hence $\pi_{\text {easy }}(\mathfrak{R} ; Y)=\pi_{\mathrm{LR}}(\mathfrak{R} ; Y)$ if $\mathfrak{R}$ is portrait, etc.) If a path $\pi$ crosses a box $\mathfrak{R}$ in the easy (hard) direction, we say that $\pi$ is an easy-crossing (hard-crossing) of $\mathfrak{R}$, and we say that $\pi$ easy-crosses (hard-crosses) $\mathfrak{R}$. Define for all $x, y \in \mathfrak{R}$

$$
\Psi_{x, y}(\mathfrak{R} ; Y)=\min _{\pi} \psi(\pi ; Y),
$$

where the minimum is taken over all paths $\pi$ connecting $x$ and $y$ while remaining inside $\mathfrak{R}$. Finally, put

$$
\Psi_{\partial}(\mathfrak{R} ; Y)=\max _{x, y \in \partial \mathfrak{B}} \Psi_{x, y}(\mathfrak{R} ; Y), \quad \text { and } \quad \Psi_{\max }(\mathfrak{R} ; Y)=\max _{x, y \in \mathfrak{B}} \Psi_{x, y}(\mathfrak{R} ; Y) .
$$

We have now several times defined symbols of the form $\Psi_{\bullet}(\Re ; Y)$ as the minimum of $\psi(\cdot ; Y)$ over some collection of paths. In each case, let $\pi_{\bullet}(\Re ; Y)$ be the path that achieves the minimum; if there are multiple such paths (which will almost surely not happen if the random variables defining the field have a sufficiently continuous distribution), let one be chosen uniformly at random, independently of everything else. We also need notation for the quantile functions for these variables, so let

$$
\Theta_{\bullet}(\Re ; Y)[p]=\inf \{w \mid \mathbf{P}[\Psi \bullet(\Re ; Y) \leq w] \geq p\} .
$$

For a path $\pi$, let $|\pi|$ denote the length of $\pi$ (i.e., the number of vertices in $\pi$ ). For $S$ a power of 2 (less than the side-length of $\mathfrak{R}$ ), let $\|\pi\|_{S}$ denote the number of dyadic square boxes of side-length $S$ entered by $\pi$, counting each box once, even if $\pi$ enters it multiple times. Let $M_{\bullet}: S(\Re ; Y)=\left\|\pi_{\bullet}(\Re ; Y)\right\|_{S}$.

Whenever the field is omitted in the $\Psi$ or $\Theta$ notation, it will be assumed to be the Gaussian free field on the box in question, defined as in the Introduction as the discrete Gaussian free field with Dirichlet boundary conditions on the boundary of the blow-up of the box. 
2.1.3. Asymptotics. Big- $O$, little- $o$, big- $\Omega$ and little- $\omega$ notation will be employed, always with the limit taken as $\gamma \rightarrow 0$. [We recall that we write $f(x)=$ $\Omega(g(x))$ if $g(x)=O(f(x))$ and $f(x)=\omega(g(x))$ if $g(x)=o(f(x))$.] Subscripts will be employed to indicate that the limit holds for any fixed value of the variable(s) in the subscript, and uniformly in all other variables. (For example, we could write $\sin \left(2^{K} \gamma\right)=o_{K}(1)$.) Most importantly, the limit is always uniform over all scales. We will also work with many constants throughout the proofs. The important point regarding any constant is that it is independent of the scale. Constants that will be referenced in later sections will be denoted by a mnemonic subscript.

2.2. Properties of the field. While we have stated our results for first-passage percolation on the discrete Gaussian free field, we do not require any particularly fine properties of this field. In this section we collect the necessary facts about the DGFF, and summarize them in Criteria 2.1-2.5. However, before we can do this we first must precisely define the DGFF, in particular the relationship between the DGFF defined on different boxes.

2.2.1. Coupling of fields in different boxes. Although we defined the discrete Gaussian free field on a box in the Introduction, in order to perform the multiscale analysis we use in this article it will be convenient to couple the fields on all different finite boxes in $\mathbf{Z}^{2}$ simultaneously. We recall the Markov field property of the Gaussian free field: that if $Y$ is a Gaussian free field with Dirichlet boundary conditions on $\mathfrak{B}$, then $Y_{\mathfrak{B}}-\mathbf{E}\left[Y_{\mathfrak{B}} \mid\left(Y_{\mathfrak{B}} \uparrow \partial \overline{\mathfrak{A}}\right)\right]$ defines a discrete Gaussian free field with Dirichlet boundary conditions on $\overline{\mathfrak{A}}$, which moreover is independent of $Y_{\mathfrak{B}}\lceil(\overline{\mathfrak{B}} \backslash \overline{\mathfrak{A}})$. Here $\lceil$ denotes restriction of the field.

Let $\mathfrak{B}_{N}=[-N, N]^{2}$. Let $Y_{\mathfrak{B}_{N}}^{(N)}$ be a discrete Gaussian free field with Dirichlet boundary conditions on $\overline{\mathfrak{B}_{N}}$. Now for all boxes $\mathfrak{B} \subset \mathfrak{B}_{N}$, for each $x \in \mathfrak{B}$ define $Y_{\mathfrak{B}}^{(N)}(x)=Y_{\mathfrak{B}_{N}}^{(N)}(x)-\mathbf{E}\left[Y_{\mathfrak{B}_{N}}^{(N)}(x) \mid\left(Y_{\mathfrak{B}_{N}}^{(N)}\lceil\partial \overline{\mathfrak{B}})\right]\right.$. Now we note that whenever $N^{\prime} \geq$ $N$, the process $\left\{Y_{\mathfrak{B}}^{(N)} \mid \mathfrak{B} \subset \mathfrak{B}_{N}\right\}$ agrees in law with the process $\left\{Y_{\mathfrak{B}}^{\left(N^{\prime}\right)} \mid \mathfrak{B} \subset \mathfrak{B}_{N}\right\}$. Indeed, if we put $Y_{\mathfrak{B}_{N}}^{(N)}$ and $Y_{\mathfrak{B}_{N^{\prime}}}^{\left(N^{\prime}\right)}$ on the same probability space so that $Y_{\mathfrak{B}_{N}}^{(N)}=$ $Y_{\mathfrak{B}_{N}}^{\left(N^{\prime}\right)}$, then for all $x \in \mathfrak{B}$ we have

$$
\begin{aligned}
& Y_{\mathfrak{B}}^{(N)}(x)=Y_{\mathfrak{B}_{N}}^{(N)}(x)-\mathbf{E}\left[Y_{\mathfrak{B}_{N}}^{(N)}(x) \mid\left(Y_{\mathfrak{B}_{N}}^{(N)} \uparrow \partial \overline{\mathfrak{B}}\right)\right] \\
& =Y_{\mathfrak{B}_{N}}^{\left(N^{\prime}\right)}(x)-\mathbf{E}\left[Y_{\mathfrak{B}_{N}}^{\left(N^{\prime}\right)}(x) \mid\left(Y_{\mathfrak{B}_{N}}^{\left(N^{\prime}\right)}\lceil\partial \overline{\mathfrak{B}})\right]\right. \\
& =Y_{\mathfrak{B}_{N^{\prime}}}^{\left(N^{\prime}\right)}(x)-\mathbf{E}\left[Y_{\mathfrak{B}_{N^{\prime}}}^{\left(N^{\prime}\right)}(x) \mid\left(Y_{\mathfrak{B}_{N^{\prime}}}^{\left(N^{\prime}\right)} \uparrow \partial \overline{\mathfrak{B}_{N}}\right)\right] \\
& -\mathbf{E}\left[Y_{\mathfrak{B}_{N^{\prime}}}^{\left(N^{\prime}\right)}(x)-\mathbf{E}\left[Y_{\mathfrak{B}_{N^{\prime}}}^{\left(N^{\prime}\right)}(x) \mid\left(Y_{\mathfrak{B}_{N^{\prime}}}^{\left(N^{\prime}\right)} \uparrow \partial \overline{\mathfrak{B}_{N}}\right)\right] \mid\left(Y_{\mathfrak{B}_{N}}^{\left(N^{\prime}\right)} \uparrow \partial \overline{\mathfrak{B}}\right)\right] \\
& =Y_{\mathfrak{B}_{N^{\prime}}}^{\left(N^{\prime}\right)}(x)-\mathbf{E}\left[Y_{\mathfrak{B}_{N^{\prime}}}^{\left(N^{\prime}\right)}(x) \mid\left(Y_{\mathfrak{B}_{N}}^{\left(N^{\prime}\right)}\lceil\partial \overline{\mathfrak{B}})\right]\right. \\
& =Y_{\mathfrak{B}}^{\left(N^{\prime}\right)}(x) \text {, }
\end{aligned}
$$


where the second-to-last equality is by the tower property of conditional expectation and the independence statement in the Markov field property. Thus, since all of the processes are Gaussian, using Kolmogorov's extension theorem we can, on a single probability space, simultaneously define $Y_{\mathfrak{B}}$ for every $\mathfrak{B} \in \mathscr{B}$ in such a way that whenever $\mathfrak{A} \subset \mathfrak{B}$, we have, for all $x \in \mathfrak{A}$,

$$
Y_{\mathfrak{A}}(x)=Y_{\mathfrak{B}}(x)-\mathbf{E}\left[Y_{\mathfrak{B}}(x) \mid\left(Y_{\mathfrak{B}} \uparrow \partial \overline{\mathfrak{A}}\right)\right] .
$$

Henceforth, we will assume that the DGFFs on different boxes have been coupled in this way, so that in particular (2.1) holds.

2.2.2. Description of the criteria for the field. Throughout the paper, we will consider a collections of real-valued random variables (the "field"), denoted $\left\{Y_{\mathfrak{B}}(x): \mathfrak{B} \in \mathscr{B}, x \in \mathfrak{B}\right\}$, and we will always assume the following five properties.

CRITERION 2.1. The field $\left\{Y_{\mathfrak{B}}(x) \mid \mathfrak{B} \in \mathscr{B}, x \in \mathfrak{B}\right\}$ is a centered Gaussian process which moreover is nonnegatively correlated: for all $\mathfrak{B}_{1}, \mathfrak{B}_{2} \in \mathscr{B}, x_{1} \in \mathfrak{B}_{1}$, $x_{2} \in \mathfrak{B}_{2}$, we have $\operatorname{Cov}\left(Y_{\mathfrak{B}_{1}}\left(x_{1}\right), Y_{\mathfrak{B}_{2}}\left(x_{2}\right)\right) \geq 0$.

CRITERION 2.2. If $\theta$ is a Euclidean isometry of $\mathbf{R}^{2}$ which preserves $\mathbf{Z}^{2}$, then the indexed families of random variables $\left\{Y_{\mathfrak{B}}(x) \mid \mathfrak{B} \in \mathscr{B}, x \in \mathfrak{B}\right\}$ and $\left\{Y_{\theta(\mathfrak{B})}(\theta(x)) \mid \mathfrak{B} \in \mathscr{B}, x \in \mathfrak{B}\right\}$ agree in distribution.

CRITERION 2.3. If $\overline{\mathfrak{B}_{1}}$ and $\overline{\mathfrak{B}_{2}}$ are disjoint, then $Y_{\mathfrak{B}_{1}}$ and $Y_{\mathfrak{B}_{2}}$ are independent.

CRIterion 2.4. There are constants $C, C_{\mathrm{F}}>0$ so that if $\mathfrak{A} \subset \mathfrak{B}$ are nested rectangles, then we have, for all $u \geq 0$,

$$
\mathbf{P}\left(\max _{x \in \mathfrak{A}}\left|Y_{\mathfrak{A}}(x)-Y_{\mathfrak{B}}(x)\right| \geq C_{\mathrm{F}}+u\right) \leq \exp \left(-\frac{C u^{2}}{\log |\mathfrak{B} / \mathfrak{A}|}\right) .
$$

CRITERION 2.5. There is an absolute constant $C$ so that the following holds. For each rectangle $\mathfrak{B}$ with a partition of its blow-up $\overline{\mathfrak{B}}=\bigsqcup_{i=1}^{r} \mathfrak{B}_{i}$ into squares $\mathfrak{B}_{i}$ of uniform side-length $S$, there is a stochastic process $\left\{Z_{i}\right\}_{i=1}^{r}$ so that $Z_{1}, \ldots, Z_{r}$ are independent, $Y_{\mathfrak{B}}(x) \in \sigma\left(Z_{1}, \ldots, Z_{r}\right)$ for all $x \in \mathfrak{B}$, and whenever $1 \leq j \leq r$ and $x \in \mathfrak{B} \backslash \overline{\mathfrak{B}_{j}}$, we have

$$
\begin{aligned}
\operatorname{Var}\left(Y_{\mathfrak{B}}(x) \mid Z_{1}, \ldots, \widehat{Z_{j}}, \ldots, Z_{r}\right) & \leq C, \\
\operatorname{Var}\left(Y_{\mathfrak{B}}(x)-Y_{\mathfrak{B}}(y) \mid Z_{1}, \ldots, \widehat{Z}_{j}, \ldots, Z_{r}\right) & \leq C\|x-y\|^{2} S^{2} / N^{4},
\end{aligned}
$$

where the hat means that $Z_{j}$ is excluded and $N$ is the length of the shorter side of $\mathfrak{B}$. 
REMARK 2.6. In the definition and use of the Markov field property above, we considered $Y_{\mathfrak{B}}(x)$ for $x \in \overline{\mathfrak{B}}$ (i.e., not in $\mathfrak{B}$ itself). This was important for defining the coupling but in the sequel we will only consider the values of $Y_{\mathfrak{B}}$ on $\mathfrak{B}$ itself.

REMARK 2.7. Although, in order to avoid the complexity of multiple cases, we will not consider this case in detail, we invite the reader to check that all of the arguments in the paper go through as well for continuous approximations of the GFF: that is, fields $\left\{Y_{\mathfrak{B}}(x): \mathfrak{B} \in \mathfrak{B}, x \in \mathfrak{B}\right\}$ satisfying Criteria 2.1-2.5, where the weight of a (continuous) path $\xi:[0,1] \rightarrow \mathfrak{B}$ is given by

$$
\int_{0}^{1} e^{\gamma Y_{\mathfrak{B}}(\xi(t))}\left|\xi^{\prime}(t)\right| d t
$$

In fact, certain technical parts of the argument (such as one case in the proof of Lemma 5.4, and the linear interpolation given in (8.1) in the sequel) become unnecessary in the continuous case.

2.2.3. Proof that the DGFF satisfies the criteria. We now demonstrate that the DGFF indeed satisfies the criteria that we have just laid out. (A much gentler introduction to these properties is available in [6].) Coupled as above, the DGFF satisfies Criteria 2.1 and 2.2. To show Criterion 2.4 for the DGFF, we first note that, by Fernique's criterion (see [19] and [1], Theorem 4.1, or [6], Theorem 6.6) and a covariance estimate on the conditional expectation field, as in [8], Lemmas 3.5 and 3.10 , we have a constant $C_{\mathrm{F}}$ so that

$$
\mathbf{E}\left[\max _{x \in \mathfrak{A}} \mathbf{E}\left[Y_{\mathfrak{B}}(x) \mid Y_{\mathfrak{B}}\lceil\partial \overline{\mathfrak{A}}]\right]<C_{\mathrm{F}}\right.
$$

Moreover, the variance of $\mathbf{E}\left[Y_{\mathfrak{B}}(x) \mid Y_{\mathfrak{B}} \uparrow \partial \overline{\mathfrak{A}}\right]$ can be bounded (uniformly over $x \in \mathfrak{A}$ ) by a constant times $\log |\mathfrak{B} / \mathfrak{A}|$. These two facts, along with the Borell-TIS inequality (see, e.g., [27], Theorem 7.1, [6], Theorem 6.1, or [1], Theorem 2.1) imply that

$$
\mathbf{P}\left(\max _{x \in \mathfrak{A}} \mathbf{E}\left[Y_{\mathfrak{B}}(x) \mid Y_{\mathfrak{B}}\lceil\partial \overline{\mathfrak{A}}] \geq C_{\mathrm{F}}+u\right) \leq \exp \left(-\frac{C u^{2}}{\log |\mathfrak{B} / \mathfrak{A}|}\right) .\right.
$$

Finally, we will prove Criterion 2.5 using the "resistor" definition of the DGFF (see, e.g., [28], page 52). For each edge $e$ in the nearest-neighbor graph on $\mathfrak{B}$, let $\xi(e)$ be a standard normal random variable, independent from $\xi\left(e^{\prime}\right)$ for each $e^{\prime} \neq e$. Then, as in [28], (2.25), we have the alternative definition of Gaussian free field on $\mathfrak{B}$ as

$$
Y_{\mathfrak{B}}(x)=\sum_{e} i_{x}(e) \xi(e)
$$


where $i_{x}(e)$ is the flow through $e$ of a unit electric current from $x$ to $\partial \overline{\mathfrak{B}}$, where the lattice is treated as an electrical network with unit resistance on each edge. Let $Z_{i}=\left(i_{x}(e): e \in \mathfrak{B}_{i}\right)$. Now if $x \in \mathfrak{B}$, we have

$$
\operatorname{Var}\left(Y_{\mathfrak{B}}(x) \mid Z_{1}, \ldots, \widehat{Z_{j}}, \ldots, Z_{r}\right)=\sum_{e \in \mathfrak{B}_{j}}\left(i_{x}(e)\right)^{2} .
$$

By [28], Proposition 2.2, we have

$$
i_{x}(e)=\frac{G_{\overline{\mathfrak{B}}}\left(x, e_{+}\right)}{\operatorname{deg}\left(e_{+}\right)}-\frac{G_{\overline{\mathfrak{B}}}\left(x, e_{-}\right)}{\operatorname{deg}\left(e_{-}\right)},
$$

So

$$
\left|i_{x}(e)\right|=\frac{1}{4}\left|G_{\overline{\mathfrak{B}}}\left(x, e_{+}\right)-G_{\overline{\mathfrak{B}}}\left(x, e_{-}\right)\right|,
$$

where $e_{-}$and $e_{+}$denote the two endpoints of $e$ and $G_{\bar{B}}$ denotes the Green's function for simple random walk stopped on the boundary of $\overline{\mathfrak{B}}$. But by [22], Proposition 4.6.2(b), Theorem 4.4.4, we have

$$
G_{\overline{\mathfrak{B}}}(x, y)=\mathbf{E}^{x}\left[a\left(Q_{\tau_{\overline{\mathfrak{B}}}}, y\right)\right]-a(x, y),
$$

where $\left\{Q_{t}\right\}$ is a simple random walk, $\tau_{\overline{\mathfrak{B}}}$ is the hitting time of $\partial \overline{\mathfrak{B}}, \mathbf{E}^{x}$ is the expectation with respect to the law of $\left\{Q_{t}\right\}$ started at $x$, and

$$
a(x, y)=\frac{2}{\pi} \log |x-y|+\frac{2 C_{1}+\log 8}{\pi}+O\left(|x-y|^{-2}\right),
$$

where $C_{1} \approx 0.577$ is the Euler-Mascheroni constant (usually denoted $\gamma$ ) and the big- $O$ notation is taken as $x-y \rightarrow \infty$. An easy computation implies that, if $x \in$ $\mathfrak{B} \backslash \overline{\mathfrak{B}_{j}}$, then $\left|i_{x}(e)\right| \leq C_{2} / S$ for some constant $C_{2}$, where $S$ is the side-length of $\mathfrak{B}_{j}$. Combining this with (2.4) shows that $\operatorname{Var}\left(Y_{\mathfrak{B}}(x) \mid Z_{1}, \ldots, \widehat{Z_{j}}, \ldots, Z_{r}\right)$ is bounded by a constant for all $x \in \mathfrak{B} \backslash \overline{\mathfrak{B}_{j}}$. This completes the proof of Criterion 2.5 for DGFF.

Finally, calculations similar to those in the proofs of Corollary 4.4 .5 and Lemma 6.3.3 in [22] show that there is a constant $C_{3}$ so that if $|x-y|=1$, then

$$
\begin{aligned}
\left|i_{x}(e)-i_{y}(e)\right| & =\frac{1}{4}\left|G_{\overline{\mathfrak{B}}}\left(x, e_{+}\right)-G_{\overline{\mathfrak{B}}}\left(x, e_{-}\right)-G_{\overline{\mathfrak{B}}}\left(y, e_{+}\right)+G_{\overline{\mathfrak{B}}}\left(y, e_{-}\right)\right| \\
& \leq \frac{C_{3}}{N^{2}},
\end{aligned}
$$

where as above $N$ is the length of the shorter side of $\mathfrak{B}$. By the triangle inequality, we then have that for any $x, y \in \mathfrak{B}$,

$$
\left|i_{x}(e)-i_{y}(e)\right| \leq C_{3} \frac{\|x-y\|}{N^{2}} .
$$


Therefore, we have

$$
\begin{aligned}
\operatorname{Var}\left(Y_{\mathfrak{B}}(x)-Y_{\mathfrak{B}}(y) \mid Z_{1}, \ldots, \widehat{Z_{j}}, \ldots, Z_{r}\right) & =\sum_{e \in \mathfrak{B}_{j}}\left(i_{x}(e)-i_{y}(e)\right)^{2} \\
& \leq C_{3}^{2}\|x-y\|^{2} S^{2} / N^{4}
\end{aligned}
$$

establishing (2.3).

2.2.4. Further properties of the field. We now record some important consequences of Criteria 2.1-2.5 that we will use throughout the paper. The first is a translation of Criterion 2.4 into the exponentiated setting. Indeed, Criterion 2.4 implies that

$$
\max _{x \in \mathfrak{A}}\left|\frac{e^{\gamma Y_{\mathfrak{B}}(x)}}{e^{\gamma Y_{\mathfrak{A}}(x)}}\right|=1+o(1)
$$

in probability (as $\gamma \rightarrow 0$ ). More precisely, there is an absolute constant $u_{0}>1$ so that if $u \geq u_{0}$ then we have

$$
\begin{gathered}
\mathbf{P}\left(\max _{x \in \mathfrak{A}}\left|\frac{e^{\gamma Y_{\mathfrak{B}}(x)}}{e^{\gamma Y_{\mathfrak{A}}(x)}}\right| \geq u\right)+\mathbf{P}\left(\max _{x \in \mathfrak{A}}\left|\frac{e^{\gamma Y_{\mathfrak{B}}(x)}}{e^{\gamma Y_{\mathfrak{A}}(x)}}\right| \leq \frac{1}{u}\right) \\
\quad=\mathbf{P}\left(\max _{x \in \mathfrak{A}}\left|\gamma Y_{\mathfrak{B}}(x)-\gamma Y_{\mathfrak{A}}(x)\right| \geq \log u\right) \\
\leq \exp \left(-\omega(1) \cdot \frac{(\log u)^{2}}{\log |\mathfrak{B} / \mathfrak{A}|}\right) .
\end{gathered}
$$

Another key ingredient, implied by Criterion 2.1, is the celebrated FKG inequality.

LEMMA 2.8 (FKG inequality). If $f$ and $g$ are increasing functions of $Y=$ $\left\{Y_{\mathfrak{B}}(x) \mid \mathfrak{B} \in \mathscr{B}, x \in \mathfrak{B}\right\}$, then

$$
\mathbf{E} f(Y) g(Y) \geq \mathbf{E} f(Y) \mathbf{E} g(Y) .
$$

See [35] for a proof of the FKG inequality for general Gaussian processes with nonnegative correlations, of which (2.7) is an application. The following corollary is typical of our applications of the FKG inequality.

COROLlary 2.9. Let $a>b$ and $S, k \in \mathbf{N}$, and put $\mathfrak{A}=[0, a S) \times[0, b S)$ and $\mathfrak{B}=[0,(k a-(k-1) b) S) \times[0, b S)$. Then

$$
\mathbf{P}\left[\Psi_{\mathrm{LR}}(\mathfrak{B}) \leq 2 k y\right] \geq \mathbf{P}\left[\Psi_{\mathrm{LR}}(\mathfrak{A}) \leq y\right]^{2 k-1}-o_{k}(1) .
$$

PROOF. This follows from (2.5) and the FKG inequality by a "gluing argument," illustrated in Figure 2. 


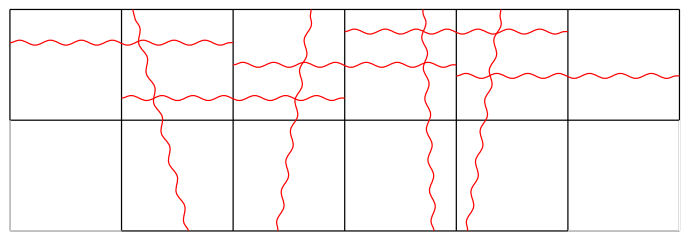

FIG. 2. Gluing strategy in Corollary 2.9 for $a=2 b$ and $k=5$.

3. Inductive hypothesis. The key ingredient for all of our results is an inductive bound on the coefficient of variation for the FPP crossing distance of a rectangle. For any random variable $X$, we define the notation

$$
\mathrm{CV}^{2}(X)=\frac{\operatorname{Var} X}{(\mathbf{E} X)^{2}} .
$$

THEOREM 3.1. Let $\delta>0$. If $\gamma$ is sufficiently small compared to $\delta$, then for all boxes $\Re$ of aspect ratio between $1 / 2$ and 2 inclusive, we have $\mathrm{CV}^{2}\left(\Psi_{\mathrm{LR}}(\mathfrak{R})\right)<\delta$.

The bulk of the paper will be devoted to proving Theorem 3.1 by induction on the scale. Actually, we will have to use the slightly stronger inductive hypothesis that the coefficient of variation is below a fixed $\delta_{0}$. The following lemma, which is an easy consequence of Chebyshev's inequality, will be key to our induction.

Lemma 3.2. Let $X$ and $Y$ be nonnegative random variables. Define $F_{X}(x)=$ $\mathbf{P}(X \leq x)$ and $F_{Y}(y)=\mathbf{P}(Y \leq y)$; let $\Theta_{X}=F_{X}^{-1}$ and $\Theta_{Y}=F_{Y}^{-1}$ be the corresponding quantile functions. If $\mathrm{CV}^{2}(X)<\delta<p<1$ and $\mathrm{CV}^{2}(Y)<\varepsilon<q<1$, then there are constants $0<A \leq B$, depending only on $\delta, \varepsilon, p, q$ (and not on the random variables $X, Y$ ) so that

$$
A \cdot \frac{\Theta_{X}(p)}{\Theta_{Y}(q)} \leq \frac{\mathbf{E} X}{\mathbf{E} Y} \leq B \cdot \frac{\Theta_{X}(p)}{\Theta_{Y}(q)} .
$$

Suppose moreover that $\delta<p^{\prime}$ and $\varepsilon<q^{\prime}$. Then there is a constant $B^{\prime}>0$, depending only on $\delta, \varepsilon, p, q, p^{\prime}, q^{\prime}$, so that

$$
\frac{\Theta_{X}\left(p^{\prime}\right)}{\Theta_{Y}\left(q^{\prime}\right)} \leq B^{\prime} \cdot \frac{\Theta_{X}(p)}{\Theta_{Y}(q)} .
$$

While the statement of Lemma 3.2 is rather involved, the content of the lemma is simply that upper bounds on the coefficients of variation of two random variables let us multiplicatively relate nonextreme quantiles and means of the random variables. 
4. Crossing quantile lower bounds. Our goal in this section is to obtain lower bounds on quantiles of the left-right crossing distance of a large box in terms of the easy crossing quantiles of smaller boxes. We first define and introduce basic properties of what we call passes, which represent smaller boxes through which a path through a larger box must cross.

Let $K, L \geq 2$ and let $S=2^{s}$. Let $\mathfrak{R}=[0, K S) \times[0, L S)$.

Definition 4.1. A pass $\mathfrak{P}$ of $\mathfrak{R}$ at scale $S$ is a $2 S \times S$ or $S \times 2 S$ dyadic subrectangle of $\mathfrak{R}$.

LEMMA 4.2. Let $\pi$ be a left-right crossing of $\mathfrak{R}$. If $\pi$ enters an $S \times S$ box $\mathfrak{C}$ such that $\overline{\mathfrak{C}} \subseteq \Re$, then $\pi$ must easy-cross a pass that intersects $\overline{\mathfrak{C}}$.

PROOF. Since $\pi$ is a left-right crossing of $\mathfrak{R}, \pi$ must at some point leave $\overline{\mathfrak{C}}$. And it is easy to see that in order to easy-cross from the inside to the outside of the annulus $\overline{\mathfrak{C}} \backslash \mathfrak{C}, \pi$ must easy-cross a pass intersecting $\overline{\mathfrak{C}}$.

DEFINITION 4.3. For a path $\pi$, let $\mathcal{P}(\pi)$ be a maximum-size collection of passes $\mathfrak{P}$ easy-crossed by $\pi$ such that the $\overline{\mathfrak{P}}$ s are disjoint (see Figure 3 ). For $N \leq$ $|\mathcal{P}(\pi)|$, let $\mathcal{P}_{N}(\pi)=\mathcal{P}(\xi)$ where $\xi$ is the minimal initial subpath of $\pi$ such that $|\mathcal{P}(\xi)| \geq N$

PROPOSITION 4.4. There is a constant $c_{\mathrm{PD}}$ so that $|\mathcal{P}(\pi)| \geq c_{\mathrm{PD}}\|\pi\|_{S}$. (The subscript stands for "pass density.")

PROOF. This follows easily from Lemma 4.2 and the fact that passes are of a fixed size.

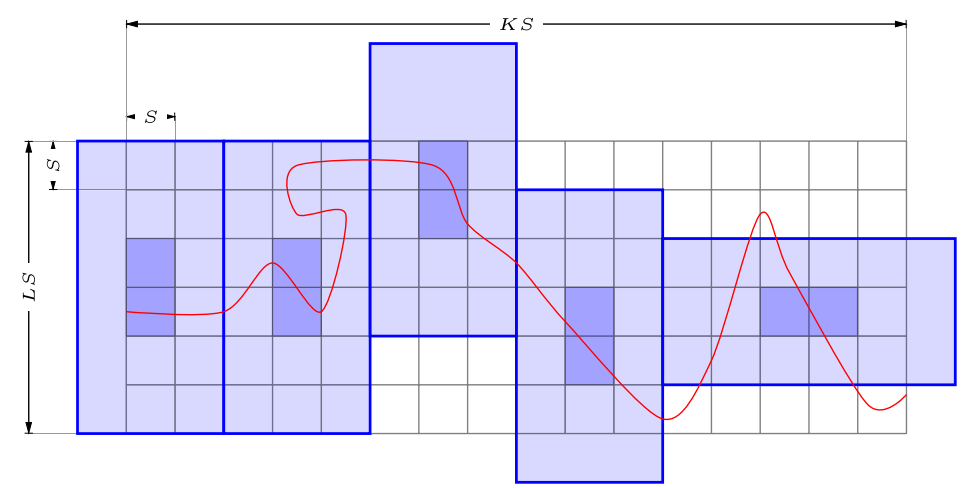

FIG. 3. $\mathcal{P}(\pi)$ for a crossing $\pi$. The darker boxes are the $\mathfrak{P}_{i} s$ while the lighter, surrounding boxes are the $\overline{\mathfrak{P}_{i}}$ s. 
LEMMA 4.5. If $\pi$ is a left-right crossing of $\Re$, then $|\mathcal{P}(\pi)| \geq K / 6$.

PROOF. In order for $\pi$ to cross each column of width $S$, it must easy-cross a pass contained entirely within that column, and the blow-up of this pass can have width at most 6 .

LEMMA 4.6. Let $G$ be a graph of maximum degree $d$, and let $\left\{a_{1}, \ldots, a_{M}\right\}$ be an arbitrary subset of the vertices of $G$. Then the number of n-vertex connected subgraphs $H$ of $G$ containing at least one $a_{i}$ is at most $M d^{2 n}$.

PROOF. It is easy to see that every connected graph on $n$ vertices contains a circuit of length at most $2 n$ that visits every vertex. Thus the number of subgraphs $H$ as specified in the statement is bounded by the number of walks of length at most $2 n$ starting at one of the $a_{i} \mathrm{~s}$, which is evidently bounded by $M d^{2 n}$.

Proposition 4.7. We have constants $C_{\mathrm{p}}$ and $d_{\mathrm{p}}$ so that

$$
\mid\left\{\mathcal{P}_{N}(\pi): \pi \text { a left-right crossing of } \mathfrak{R} \text { such that }|\mathcal{P}(\pi)| \geq N\right\} \mid \leq C_{\mathrm{p}} L d_{\mathrm{p}}^{2 N} \text {. }
$$

Proof. Define a graph $G$ on the set of all passes inside $\mathfrak{R}$ by saying that two passes are adjacent if they could occur as adjacent passes in a $\mathcal{P}(\pi)$. It is easy to see using Lemma 4.2 that $G$ has bounded degree. Then by definition, $\mathcal{P}_{N}(\pi)$ induces an $N$-element connected subgraph of $G$, which in particular contains a pass that intersects the first six columns, of which there are at most a constant times $L$. Lemma 4.6 then implies the desired result.

Before we prove the main proposition of this section we need a version of the Chernoff bound.

LEMMA 4.8. Let $p<\frac{1}{2}$ and $X_{1}, \ldots, X_{N}$ be i.i.d. Bernoulli( $\left.p\right)$ random variables. Then $\mathbf{P}\left[\frac{1}{N} \sum_{i=1}^{N} X_{i} \geq \frac{1}{2}\right] \leq(4 p)^{N / 2}$.

Proof. We have

$$
\begin{aligned}
\mathbf{P}\left[\frac{1}{N} \sum_{i=1}^{N} X_{i} \geq \frac{1}{2}\right] & =\mathbf{P}\left[\exp \left(\lambda \cdot \sum_{i=1}^{N} X_{i}\right) \geq e^{\lambda N / 2}\right] \leq \frac{\left(\mathbf{E} e^{\lambda X_{i}}\right)^{N}}{e^{\lambda N / 2}} \\
& =\left(\frac{p e^{\lambda}+1-p}{e^{\lambda / 2}}\right)^{N} .
\end{aligned}
$$

Putting $\lambda=\log \frac{1-p}{p}$ and using the fact that $p<1 / 2$ yields the result.

Now we can prove an inductive lower bound on the crossing LFPP distance. 
Proposition 4.9. Let $S=2^{s}$ and let $K, L \in \mathbf{N}$. Let $\mathfrak{R}=[0, K S) \times[0, L S)$ and $\mathfrak{A}=[0, S) \times[0,2 S)$. Then, for any $p \in(0,1 / 2)$ and any $u \geq u_{0}$ (defined in (2.6)), we have

$$
\begin{aligned}
& \mathbf{P}\left[\min _{\pi} \psi\left(\pi ; Y_{\mathfrak{R}}\right) \leq \frac{N}{2 u} \Theta_{\text {easy }}(\mathfrak{A})[p]\right] \\
& \quad \leq C_{\mathrm{p}} L\left(2 d_{\mathrm{p}}^{2} \sqrt{p}\right)^{N}+K L \exp \left(-\omega(1) \cdot \frac{(\log u)^{2}}{\log (K \vee L)}\right),
\end{aligned}
$$

where the minimum is taken over all paths $\pi$ from left to right in $\mathfrak{R}$ with $|\mathcal{P}(\pi)| \geq N$.

PROOF. As long as $\gamma$ is sufficiently small compared to $K$ and $L$, and $u \geq u_{0}$, we have

$$
\begin{aligned}
\mathbf{P}\left[\min _{\pi} \psi\left(\pi ; Y_{\mathfrak{R}}\right) \leq \frac{N}{2 u} \Theta_{\text {easy }}(\mathfrak{A})[p]\right] \\
\leq \mathbf{P}\left[\min _{\pi} \frac{1}{N} \sum_{\mathfrak{P} \in \mathcal{P}_{N}(\pi)} \mathbf{1}\left\{\Psi_{\text {easy }}\left(\mathfrak{P} ; Y_{\mathfrak{R}}\right) \leq \frac{1}{u} \Theta_{\text {easy }}(\mathfrak{A})[p]\right\} \geq \frac{1}{2}\right] \\
\leq \mathbf{P}\left[\min _{\pi} \frac{1}{N} \sum_{\mathfrak{P} \in \mathcal{P}_{N}(\pi)} \mathbf{1}\left\{\Psi_{\text {easy }}(\mathfrak{P}) \leq \Theta_{\text {easy }}(\mathfrak{A})[p]\right\} \geq \frac{1}{2}\right] \\
+K L \exp \left(-\omega(1) \cdot \frac{(\log u)^{2}}{\log (K \vee L)}\right),
\end{aligned}
$$

where in the second inequality we use (2.6) and Proposition 4.7. Now let $X_{1}, \ldots, X_{N}$ be i.i.d. copies of $\mathbf{1}\left\{\Psi_{\text {easy }}(\mathfrak{A}) \leq \Theta_{\text {easy }}(\mathfrak{A})[p]\right\}$. By Criterion 2.3 , the first term on the right-hand side of (4.1) is bounded above by

$$
C_{\mathrm{p}} L d_{\mathrm{p}}^{2 N} \mathbf{P}\left[\frac{1}{N} \sum_{i=1}^{N} X_{i} \geq \frac{1}{2}\right]
$$

which is bounded above by $C_{\mathrm{p}} L\left(2 d_{\mathrm{p}}^{2} \sqrt{p}\right)^{N}$ according to Lemma 4.8. This completes the proof.

Corollary 4.10. Fix a scale $S=2^{s}$ and let $K, L \in \mathbf{N}$. Let $\mathfrak{R}=[0, K S) \times$ $[0, L S)$ and $\mathfrak{A}=[0, S) \times[0,2 S)$. Then we have

$$
\Theta_{\mathrm{LR}}(\mathfrak{R})\left[C_{\mathrm{p}} L\left(2 d_{\mathrm{p}}^{2} \sqrt{p}\right)^{K / 3}+o_{K, L, p}(1)\right] \geq \frac{K}{12 u_{0}} \Theta_{\text {easy }}(\mathfrak{A})[p]
$$

and

$$
\mathbf{E} \Psi_{\mathrm{LR}}(\mathfrak{R}) \geq \frac{K}{12 u_{0}} \Theta_{\text {easy }}(\mathfrak{A})[p] \cdot\left(1-C_{\mathrm{p}} L\left(2 d_{\mathrm{p}}^{2} \sqrt{p}\right)^{K / 2 u_{0}}-o_{K, L, p}(1)\right) .
$$


PROOF. If $\pi$ is a path from left to right in $\mathfrak{R}$, then by Lemma 4.5 , we have $|\mathcal{P}(\pi)| \geq K / 6$. Proposition 4.9 then implies the first equation. The second equation follows immediately from the first.

We conclude this section with an inductive version of Corollary 4.10, showing that some easy crossing quantile grows like $S^{\Omega(1)}$ in the scale $S$.

PROPOSITION 4.11. There are constants $p_{\mathrm{pl}}, q_{\mathrm{pl}}, a_{\mathrm{pl}} \in(0,1)$ and a constant $C_{\mathrm{pl}}>0$ so that, if $p<p_{\mathrm{pl}}$ and $\gamma$ is sufficiently small compared to $p$, then, putting $\mathfrak{R}_{t}=\left[0,2^{t}\right) \times\left[0,2^{t+1}\right)$, for any $s>t$ we have

$$
\Theta_{\text {easy }}\left(\Re_{t}\right)\left[p_{\mathrm{pl}}\right] \leq C_{\mathrm{pl}} a_{\mathrm{pl}}^{s-t} \Theta_{\text {easy }}\left(\Re_{s}\right)\left[q_{\mathrm{pl}}\right] .
$$

(The subscript $\mathrm{pl}$ stands for "power-law.”)

PROOF. Fix a large constant $K$, to be chosen later. Write $s=t+n k+r$, where $0 \leq r<k=\log _{2} K$. Let $R=2^{r}$. We can calculate, using Corollary 4.10,

$$
\begin{aligned}
\Theta_{\text {easy }}\left(\Re_{t}\right)[p] & \leq \frac{12 u_{0}}{K} \Theta_{\text {easy }}\left(\Re_{t+k}\right)\left[2 C_{\mathrm{p}} K\left(d_{\mathrm{p}}^{2} \sqrt{p}\right)^{K / 3}+o_{K}(1)\right] \\
& \leq \frac{12 u_{0}}{K} \Theta_{\text {easy }}\left(\Re_{t+k}\right)[p]
\end{aligned}
$$

where in the second inequality we use the assumption that $p$ is sufficiently small, $K$ is sufficiently large and $\gamma$ is sufficiently small (compared to $p$ and $K$ ). By induction, we obtain

$$
\begin{aligned}
\Theta_{\text {easy }}\left(\Re_{t}\right)[p] & \leq\left(\frac{12 u_{0}}{K}\right)^{n} \Theta_{\text {easy }}\left(\Re_{t+n k}\right)[p] \\
& =\left(\frac{\left(12 u_{0}\right)^{1 / k}}{2}\right)^{k n} \Theta_{\text {easy }}\left(\Re_{t+n k}\right)[p]
\end{aligned}
$$

Thus, applying Corollary 4.10 once more, we get

$$
\begin{aligned}
\Theta_{\text {easy }}\left(\Re_{t+n k}\right)[p] & \leq \frac{12 u_{0}}{R} \Theta_{\text {easy }}\left(\Re_{t+n k+r}\right)\left[4 R\left(d_{\mathrm{p}}^{2} \sqrt{p}\right)^{R / 3}+o_{K}(1)\right] \\
& \leq \frac{12 u_{0}}{R} \Theta_{\text {easy }}\left(\Re_{t+n k+r}\right)\left[q_{\mathrm{pl}}\right]
\end{aligned}
$$

where $p, K, \gamma$ are restricted so that $q_{\mathrm{pl}}$ can be chosen to be less than 1 . Thus we get the desired inequality with $a_{\mathrm{pl}}=\left(12 u_{0}\right)^{1 / k} / 2 \in(0,1)$ as long as $K$ is sufficiently large. 
5. RSW result. We will prove the following RSW result relating easy crossings to hard crossings of $2 \times 1$ rectangles.

THEOREM 5.1. There are constants $\delta_{\mathrm{RSW}}>0, C_{\mathrm{RSW}}<\infty, p_{\mathrm{RSW}}>0$ so that

$$
p_{\mathrm{RSW}} \leq 1 /\left(32 \cdot d_{\mathrm{p}}^{2}\right)^{2}
$$

and, if $\gamma$ is sufficiently small then the following holds. Let $\mathfrak{R}=[0, S) \times[0,2 S)$. Suppose that, for all $\mathfrak{A} \subseteq \mathfrak{R}$ of aspect ratio between $1 / 2$ and 2 inclusive, we have

$$
\mathrm{CV}^{2}\left(\Psi_{\text {easy }}(\mathfrak{A})\right)<\delta_{\text {RSW }} .
$$

Then

$$
\Theta_{\text {hard }}(\Re)\left[p_{\text {RSW }}\right] \leq C_{\text {RSW }} \Theta_{\text {easy }}(\Re)\left[p_{\text {RSW }} .\right.
$$

Our argument is based on the beautiful proof of the RSW result established for Voronoi percolation in [43]. While our proof has the same structure and uses many of the same geometric constructions, two factors make our setting substantially more complicated than the Voronoi percolation case:

1. We need to take the weights of crossings into account.

2. We do not have as strong a duality theory in the first-passage percolation setting, so rather than comparing crossings for a square and a rectangle, we compare crossings for the easy and hard directions of rectangles.

While our argument is self-contained, a reader first equipped with a thorough understanding of the arguments in [43] will find it much easier to follow.

5.1. Scale and aspect ratio setup. Fix $p_{0} \in\left(0, p_{\mathrm{pl}}\right)$, with $p_{\mathrm{pl}}$ as in Proposition 4.11.

We will work with rectangles in the portrait orientation with aspect ratio $\eta=$ $1+2^{-t_{0}}$, where $t_{0}$ is fixed but will be chosen later. It will be convenient to work at a series of fixed scales where there are no rounding problems, so for $i \in \mathbf{N}$, let $u_{i}=[i / 2], U_{i}=2^{u_{i}}$ and

$$
T_{i}=2^{t_{0}+8}(3 / 2)^{2\{i / 2\}} U_{i}=256 \cdot(3 / 2)^{2\{i / 2\}} \cdot 2^{t_{0}+[i / 2]},
$$

where $[x]$ is the integer part of $x$ and $x=[x]+\{x\}$. (By way of illustration, we note that the first few terms of the sequence $T_{0}, T_{1}, T_{2}, \ldots$ are $2^{t_{0}+7}$ times $2,3,4,6,8,12, \ldots$. The factor of 256 is to accommodate occasions when we need to split up the boxes in certain constructions and is not important to the argument.) In particular, $T_{i+1} \in\left[4 T_{i} / 3,3 T_{i} / 2\right]$ for each $i$ and $\eta T_{i} \in \mathbf{N}$ for each $i$, and if $j \geq i$, we have the simple estimates

$$
\sqrt{2}^{j-i-1} T_{i} \leq T_{j} \leq \sqrt{2}^{j-i+1} T_{i} .
$$


Let $S_{i}=2^{s_{i}}=2^{t_{0}+9+\lceil i / 2\rceil}$ be the least dyadic integer greater than or equal to $T_{i}$. Let

$$
\mathfrak{R}_{i}=\left[0, S_{i}\right) \times\left[0,2 S_{i}\right), \quad \mathfrak{R}_{i}^{(\eta)}=\left[0, T_{i}\right) \times\left[0, \eta T_{i}\right)
$$

and put

$$
w_{i}^{(\eta)}=\Theta_{\text {easy }}\left(\Re_{i}^{(\eta)}\right)\left[p_{0}\right], \quad W_{i}^{(\eta)}=\max _{j \leq i} w_{i}^{(\eta)}
$$

It will be convenient to put $w_{i}^{(\eta)}=W_{i}^{(\eta)}=0$ when $i<0$.

In this section we will need notation for new types of crossing distances. Let $\mathfrak{B}$ be a box with bottom-left corner $\left(x_{0}, y_{0}\right)$ and top-right corner $\left(x_{1}, y_{1}\right)$. If $I_{1}, I_{2} \subset \mathbf{Z}$, then let $\Psi_{I_{1}, I_{2}}(\mathfrak{B} ; Y)$ denote the minimum $Y$-weight of a crossing $\pi$ in $\mathfrak{B}$ connecting $\left\{x_{0}\right\} \times\left(y_{0}+I_{1}\right)$ and $\left\{x_{1}\right\} \times\left(y_{0}+I_{2}\right)$. Let

$$
\begin{aligned}
& \Psi_{\mathrm{L}, a, b}=\Psi_{\left\{x_{0}\right\} \times\left[0, y_{1}-y_{0}\right),\left\{x_{1}\right\} \times\left(y_{0}+[a, b)\right)}, \\
& \Psi_{a, b, \mathrm{R}}=\Psi_{\left\{x_{0}\right\} \times\left(y_{0}+[a, b)\right),\left\{x_{1}\right\} \times\left[0, y_{1}-y_{0}\right)} .
\end{aligned}
$$

Finally, define $\Psi_{\mathrm{X} ; a}(\mathfrak{B} ; Y)$ to be the minimum $Y$-weight of a crossing in $\mathfrak{B}$ that connects the four segments $\left\{x_{0}\right\} \times\left[y_{0}, \frac{y_{0}+y_{1}}{2}-a\right),\left\{x_{1}\right\} \times\left[y_{0}, \frac{y_{0}+y_{1}}{2}-a\right),\left\{x_{0}\right\} \times$ $\left[\frac{y_{0}+y_{1}}{2}+a, y_{1}\right)$, and $\left\{x_{1}\right\} \times\left[\frac{y_{0}+y_{1}}{2}+a, y_{1}\right)$ (thus forming an " $\mathrm{X}$ " shape with each arm terminating at least a distance $a$ from the horizontal midline of the box). We moreover extend the $\pi$ and $\Theta$ notation accordingly as in Section 2.1. This notation is concordant with the $\mathcal{X}$ and $\mathcal{H}$ notation in [43].

We aim to prove Theorem 5.1, which is about portrait $1 \times 2$ rectangles; however, we will argue using rectangles which are portrait but very close to square. In order to conclude, we will need to relate the $w_{i}^{(\eta)}$ s and the crossing quantiles for $1 \times 2$ rectangles. We do this in the following lemma and corollary.

LEMMA 5.2. Let $S$ and $b<k$ be natural numbers and put $\mathfrak{A}=[0, b S) \times$ $[0,(b+1) S)$ and $\mathfrak{B}=[0, b S) \times[0, k S)$. Then

$$
\mathbf{P}\left[\Psi_{\text {easy }}(\mathfrak{B}) \leq y\right] \leq 2 k \mathbf{P}\left[\Psi_{\text {easy }}(\mathfrak{A}) \leq y\right]+o_{k}(1) .
$$

ProOF. Divide the rectangle $[0, b S) \times[0, k S)$ into $k b S \times S$ landscape subrectangles. Now a left-right crossing of $[0, b S) \times[0, k S)$ must either cross horizontally within a block of $b+1$ of these subrectangles ( $k-b$ such blocks) or else cross vertically a block of $b$ of these subrectangles $(k-b+1$ such blocks). Each of these events has probability at most

$$
\mathbf{P}\left[\Psi_{\mathrm{LR}}(\mathfrak{A}) \leq y\right]+o_{|\mathfrak{B} / \mathfrak{A}|}(1)
$$

(using (2.5)) so the conclusion of the lemma follows from a union bound.

COROLlaRY 5.3. For any fixed $\eta>1$ the following holds. There are constants $C_{\mathrm{str}}(\eta)<\infty$ and $p_{\mathrm{str}}(\eta) \in(0,1)$ so that, if $\gamma$ is sufficiently small, then $w_{i}^{(\eta)} \leq C_{\text {str }}(\eta) \cdot \Theta_{\text {easy }}\left(\Re_{i}\right)\left[p_{\text {str }}(\eta)\right]$. 
5.2. Gluing. We now begin in earnest the proof of our RSW result.

LEMMA 5.4. There is a $p_{1}>0$, depending only on $p_{0}$, so that the following holds. Let $y \geq w_{i}^{(\eta)}$ and let

$$
\begin{aligned}
f_{y}(\alpha, \beta) & =\mathbf{P}\left[\Psi_{L, \alpha, \beta}\left(\Re_{i}^{(\eta)}\right) \leq y\right], \\
g_{w, y}(\alpha) & =f_{w}(0, \alpha)-f_{y}\left(\alpha, \eta T_{i} / 2\right) \quad \text { and } \\
\lambda=\lambda_{i}^{y} & =\frac{\eta T_{i}}{8} \wedge \min \left\{\alpha \in\left\{1, \ldots, \eta T_{i}\right\} \mid g_{w_{i}^{(\eta)}, y}(\alpha) \geq p_{0} / 4\right\} .
\end{aligned}
$$

Then $\lambda$ is a well-defined element of $\left[0, \eta T_{i} / 8\right]$ and the following two statements both hold:

\section{Either}

(a) $\mathbf{P}\left[\Psi_{\mathrm{LR}}\left(\left[0,2 T_{i}\right) \times\left[0, \eta T_{i}\right)\right) \leq 3 y\right] \geq p_{1}$, or

(b) $\mathbf{P}\left[\Psi_{\mathrm{L}, \lambda, \eta T_{i}}\left(\mathfrak{R}_{i}^{(\eta)}\right) \leq y\right] \geq p_{1}$.

2. If $\lambda<\eta T_{i} / 8$, then

$$
\mathbf{P}\left[\Psi_{\mathrm{L}, 0, \lambda}\left(\Re_{i}^{(\eta)}\right) \leq w_{i}^{(\eta)}\right] \geq \frac{p_{0}}{4}+\mathbf{P}\left[\Psi_{\mathrm{L}, \lambda, \eta T_{i}}\left(\Re_{i}^{(\eta)}\right) \leq y\right] .
$$

REMARK 5.5. Note that $f_{y}(\alpha, \beta)$ is increasing in $y$, so $g_{w, y}(\alpha)$ is decreasing in $y$, and thus $\lambda_{i}^{y}$ is increasing in $y$. Moreover, for each $i$, there is a $y_{i}^{*}$ so that

$$
\lambda_{i}^{y_{i}^{*}}=\eta T_{i} / 8
$$

PROOF. First note that $g_{w_{i}^{(\eta)}, y}$ is increasing, we have $g_{w_{i}^{(\eta)}, y}(0)<0$ and $g_{w_{i}^{(\eta)}, y}\left(\eta T_{i}\right)=f_{w_{i}^{(\eta)}}\left(\eta T_{i}\right) \geq p_{0} / 2$. Thus $\lambda$ is well defined by the definition in the statement of the theorem. Note that symmetry implies that, for any $\alpha \in$ $\left(0, \ldots, \eta T_{i} / 2\right)$,

$$
\begin{aligned}
p_{0} / 2 & \leq f_{w_{i}^{(\eta)}}\left(0, \eta T_{i} / 2\right) \leq f_{w_{i}^{(\eta)}}(0, \alpha)+f_{w_{i}^{(\eta)}}\left(\alpha, \eta T_{i} / 2\right) \\
& \leq f_{w_{i}^{(\eta)}}(0, \alpha)+f_{y}\left(\alpha, \eta T_{i} / 2\right)
\end{aligned}
$$

so (using (5.5))

$$
f_{w_{i}^{(\eta)}}(0, \lambda) \geq p_{0} / 4+f_{y}\left(\lambda, \eta T_{i} / 2\right) \geq p_{0} / 4
$$

whenever $\lambda<\eta T_{i} / 8$, and

$$
f_{w_{i}^{(\eta)}}(0, \lambda-1)-f_{y}\left(\lambda-1, \eta T_{i} / 2\right)<p_{0} / 4
$$

In particular, statement 2 holds. 
The proof of statement 1 comes down to two cases, depending on the value of $g_{w_{i}^{(\eta)}, y}(\lambda)$.

Case 1. If $g_{w_{i}^{(\eta)}, y}(\lambda) \geq 3 p_{0} / 8$, then this along with (5.7) implies that

$$
\begin{aligned}
p_{0} / 8 & <f_{w_{i}^{(\eta)}}(0, \lambda)-f_{y}\left(\lambda, \eta T_{i}\right)-\left[f_{w_{i}^{(\eta)}}(0, \lambda-1)-f_{y}\left(\lambda-1, \eta T_{i} / 2\right)\right] \\
& \leq f_{y}\left(\lambda-1, \eta T_{i} / 2\right)-f_{y}\left(\lambda, \eta T_{i}\right) \leq \mathbf{P}\left[\Psi_{\mathrm{L}, \lambda-1, \lambda}\left(\Re_{i}^{(\eta)}\right) \leq y\right]
\end{aligned}
$$

In words, this means that the probability of a crossing of weight at most $y$ from the left-hand side of $\mathfrak{R}_{i}^{(\eta)}$ to the point with coordinates $\left(T_{i}, \eta T_{i} / 2+\lambda-1\right)$ is at least $p_{0} / 8$. But then (using the FKG inequality and (2.5))

$$
\begin{aligned}
& \mathbf{P}\left[\Psi_{\mathrm{LR}}\left(\left[0,2 T_{i}\right) \times\left[0, \eta T_{i}\right)\right) \leq 3 y\right] \\
& \quad \geq \mathbf{P}\left[\Psi_{\mathrm{L}, \lambda-1, \lambda}\left(\Re_{i}^{(\eta)}\right) \leq y\right] \mathbf{P}\left[\Psi_{\lambda-1, \lambda, \mathrm{R}}\left(\Re_{i}^{(\eta)}\right) \leq y\right]-o(1)>\left(\frac{p_{0}}{8}\right)^{2}-o(1),
\end{aligned}
$$

so as long as $p_{1} \leq\left(p_{0} / 8\right)^{2}$, then statement 1 (a) holds.

Case 2. Now suppose $g_{w_{i}^{(\eta)}, y}(\lambda)<3 p_{0} / 8$. This means that we have

$$
\begin{aligned}
p_{0} / 2 & \leq f_{w_{i}^{(\eta)}}(0, \lambda)+f_{w_{i}^{(\eta)}}\left(\lambda, \eta T_{i} / 2\right) \leq f_{w_{i}^{(\eta)}}(0, \lambda)+f_{y}\left(\lambda, \eta T_{i} / 2\right) \\
& \leq g_{w_{i}^{(\eta)}, y}(\lambda)+2 f_{y}\left(\lambda, \eta T_{i} / 2\right) \leq 3 p_{0} / 8+2 f_{y}\left(\lambda, \eta T_{i} / 2\right),
\end{aligned}
$$

so $f_{y}\left(\lambda, \eta T_{i} / 2\right) \geq p_{0} / 16$. So as long as $p_{1}<p_{0} / 16$, statement $1(\mathrm{~b})$ holds.

LEMMA 5.6. If statement 1(b) of Lemma 5.4 holds, and $\gamma$ is sufficiently small, then there is a $p_{2}>0$, depending only on $p_{1}$, so that the following holds. Let $y \geq w_{i}^{(\eta)}$. Suppose that

$$
\eta-\frac{\lambda_{i}^{y}}{32 T_{i}}<1 .
$$

Then if

$$
\begin{aligned}
& \mu=\mu_{i}^{y} \in\left(\frac{1}{16} \lambda_{i}^{y}, \frac{1}{8} \lambda_{i}^{y}\right) \quad \text { and } \\
& \nu=v_{i}^{y}=2 \lambda_{i}^{y}-\mu_{i}^{y},
\end{aligned}
$$

then

$$
\mathbf{P}\left[\Psi_{\mathrm{X} ;(\nu-\mu) / 2}\left(\mathfrak{R}_{i}^{(\eta)}\right) \leq 9 y\right] \geq p_{2}
$$

Proof. Note that

$$
\begin{aligned}
& \left\{\Psi_{\left[\eta T_{i} / 2+\nu / 2, \eta T_{i}\right),\left[\eta T_{i} / 2+\nu / 2, \eta T_{i}\right)}\left(\mathfrak{R}_{i}^{(\eta)}\right) \leq 2 y\right\} \\
& \quad \supset\left\{\Psi_{\mathrm{L}, \nu / 2, \eta T_{i} / 2}\left(\Re_{i}^{(\eta)}\right) \leq y\right\} \cap\left\{\Psi_{\nu / 2, \eta T_{i} / 2, \mathrm{R}}\left(\Re_{i}^{(\eta)}\right) \leq y\right\} .
\end{aligned}
$$


By combining (5.11), the FKG inequality, (2.5) and statement 1(b) of Lemma 5.4, we have

$$
\begin{aligned}
\mathbf{P}\left[\Psi_{[}\right. & \left.\eta T_{i} / 2+v / 2, \eta T_{i}\right],\left[\eta T_{i} / 2+v / 2, \eta T_{i}\right] \\
& \geq \mathbf{R}(\eta)) \leq 3 y] \\
& \geq \mathbf{P}\left[\Psi_{\mathrm{L}, v / 2, \eta T_{i} / 2}\left(\mathfrak{R}_{i}^{(\eta)}\right) \leq y\right]^{2}-o(1) \\
& \geq \mathbf{P}\left[\Psi_{\mathrm{L}, \lambda, \eta T_{i} / 2}\left(\mathfrak{R}_{i}^{(\eta)}\right) \leq y\right]^{2}-o(1) \\
& \geq p_{1}^{2}-o(1) .
\end{aligned}
$$

Let $\tilde{\mathfrak{R}}_{i}^{(\eta)}=\left[0, T_{i}\right) \times\left[\mu, \eta T_{i}\right)$. By (5.8) and (5.9), $\tilde{\mathfrak{R}}_{i}^{(\eta)}$ is landscape. Let $E$ be the event that there is a left-right path in $\mathfrak{R}_{i}^{(\eta)}$ connecting the intervals $\left[\eta T_{i} / 2+\right.$ $\left.v / 2, \eta T_{i}\right)$ on each side, of weight at most $3 y$, that enters the box $\mathfrak{R}_{i}^{(\eta)} \backslash \tilde{\mathfrak{R}}_{i}^{(\eta)}$. Then we have that

$$
\begin{aligned}
& \mathbf{P}\left[\Psi_{\mathrm{L}, v / 2, \eta T_{i} / 2}\left(\tilde{\mathfrak{R}}_{i}^{(\eta)}\right) \leq 3 y\right]+\mathbf{P}[E] \\
& \quad \geq \mathbf{P}\left[\Psi_{\left[\eta T_{i} / 2+v / 2, \eta T_{i}\right],\left[\eta T_{i} / 2+v / 2, \eta T_{i}\right]}\left(\Re_{i}^{(\eta)}\right) \leq 3 y\right] \\
& \quad \geq p_{1}^{2}-o(1),
\end{aligned}
$$

where for the second inequality we use (5.12). Thus, either

$$
\mathbf{P}\left[\Psi_{\mathrm{L}, \nu / 2, \eta T_{i} / 2}\left(\tilde{\mathfrak{R}}_{i}^{(\eta)}\right) \leq 3 y\right] \geq p_{1}^{2} / 2-o(1)
$$

or

$$
\mathbf{P}[E] \geq p_{1}^{2} / 2-o(1) .
$$

We consider each case in turn.

Case 1. First suppose that (5.14) holds. Let $E_{1}=E$ and define $E_{2}$ to be a copy of $E$ which is vertically flipped and translated upward by $\mu$. Then the intersection of $E_{1}$ and $E_{2}$ is contained in $\left\{\Psi_{X ;(\nu-\mu) / 2}\left(\Re_{i}^{(\eta)}\right) \leq 6 y\right\}$. This is because the path in $E_{2}$ must cross the path from $E_{1}$ once on its way from $0 \times\left[\mu, \mu+\eta T_{i} / 2-v / 2\right)$ to $\left(\mathfrak{R}_{i}^{(\eta)}+(0, \mu)\right) \backslash \mathfrak{R}_{i}^{(\eta)}$, and another time on its way from $\left(\mathfrak{R}_{i}^{(\eta)}+(0, \mu)\right) \backslash \mathfrak{R}_{i}^{(\eta)}$ to $\left\{T_{i}-1\right\} \times\left[\mu, \mu+\eta T_{i} / 2-v / 2\right)($ see Figure 4). Thus we have

$$
\mathbf{P}\left[\Psi_{\mathrm{X} ;(v-\mu) / 2}\left(\mathfrak{R}_{i}^{(\eta)}\right) \leq 6 y\right] \geq\left(p_{1}^{2} / 2\right)^{2}-o(1)
$$

by the FKG inequality. This proves the lemma in this case, as long as $p_{2} \leq p_{1}^{4} / 4-$ $o(1)$.

Case 2. We are left with the case when (5.13) holds, which in particular means that

$$
\mathbf{P}\left[\Psi_{\mathrm{LR}}\left(\tilde{\mathfrak{R}}_{i}^{(\eta)}\right) \leq 3 y\right] \geq p_{1}^{2} / 2-o(1) .
$$




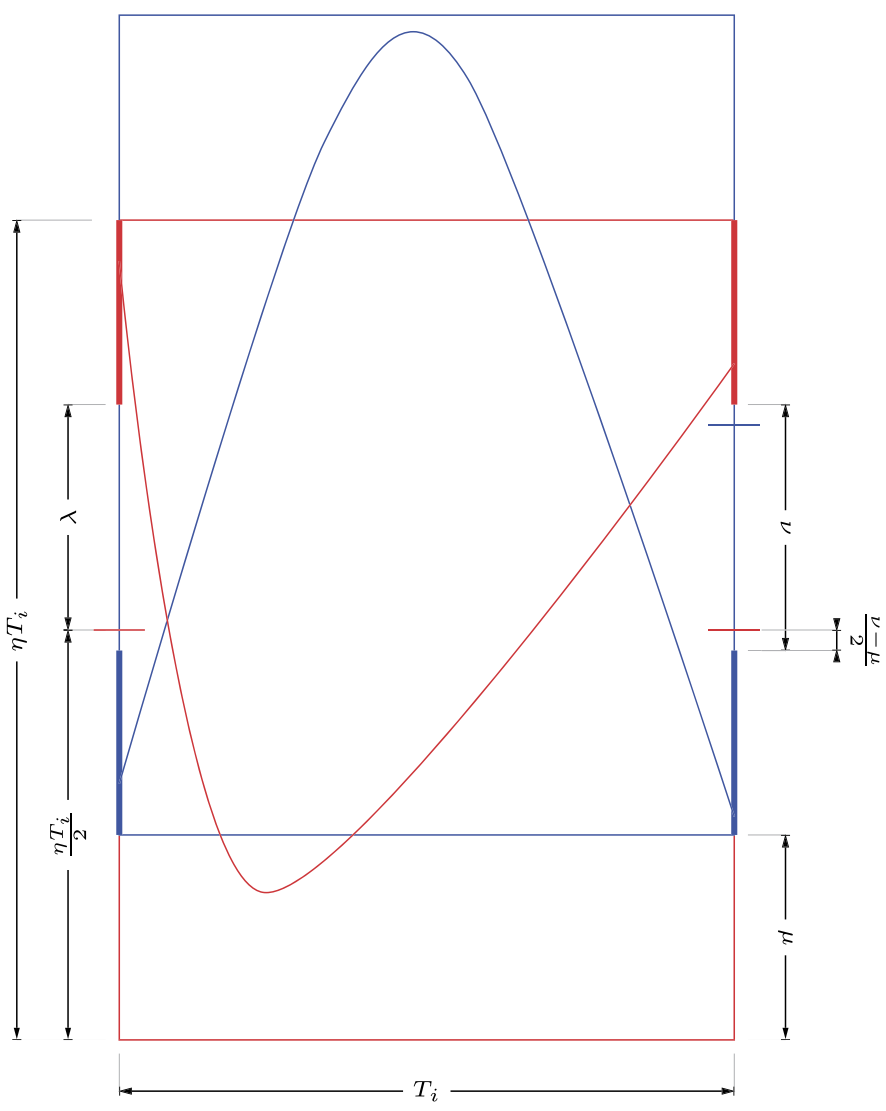

FIG. 4. Setting up for the $F K G$ inequality when $\mathbf{P}[E] \geq p_{1}^{2} / 2$. Combining the red crossing with the lower pieces of the blue crossing gives an " $X$ " shape inside the red $T_{i} \times \eta T_{i}$ box with endpoints at least distance $(v-\mu) / 2$ from the midline. In this and future figures in this section, the origin $(0,0)$ is at the bottom left.

Observe that the event $\left\{\Psi_{\mathrm{X} ; \nu / 2}\left(\mathfrak{R}_{i}^{(\eta)}\right) \leq 9 y\right\}$ contains the intersection of the three events

$$
\begin{array}{r}
\left\{\Psi_{\left[\eta T_{i} / 2+v / 2, \eta T_{i}\right),\left[\eta T_{i} / 2+v / 2, \eta T_{i}\right)}\left(\Re_{i}^{(\eta)}\right) \leq 3 y\right\}, \\
\left\{\Psi_{\left[0, \eta T_{i} / 2-v / 2\right),\left[0, \eta T_{i} / 2-v / 2\right)}\left(\Re_{i}^{(\eta)}\right) \leq 3 y\right\} \text { and } \\
\left\{\Psi_{\mathrm{BT}}\left(\Re_{i}^{(\eta)}\right) \leq 3 y\right\}
\end{array}
$$

(see Figure 5), so

$$
\mathbf{P}\left[\Psi_{\mathrm{X} ; \nu / 2}\left(\Re_{i}^{(\eta)}\right) \leq 9 y\right]
$$

(5.16) $\geq \mathbf{P}\left[\Psi_{\left[\eta T_{i} / 2+v / 2, \eta T_{i}\right),\left[\eta T_{i} / 2+v / 2, \eta T_{i}\right)}\left(\mathfrak{R}_{i}^{(\eta)}\right) \leq 3 y\right]^{2} \mathbf{P}\left[\Psi_{\mathrm{BT}}\left(\mathfrak{R}_{i}^{(\eta)}\right) \leq 3 y\right]$ 


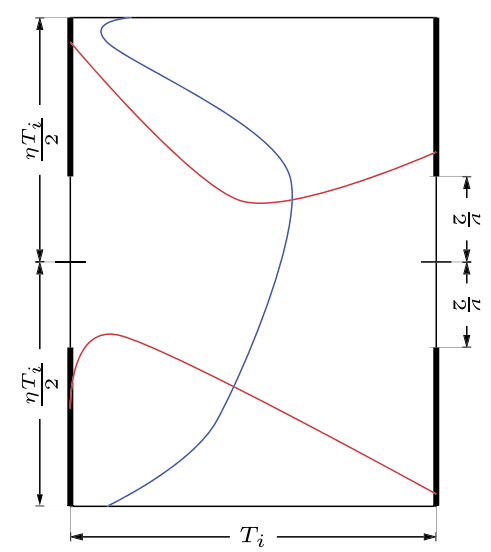

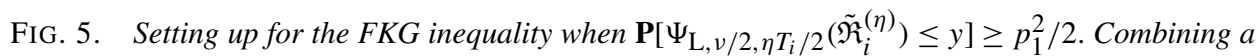
piece of the blue vertical crossing with the red horizontal crossings gives an " $X$ " shape inside the $T_{i} \times \eta T_{i}$ box with endpoints at least distance $\lambda \geq(\nu-\mu) / 2$ from the midline.

$$
\geq p_{1}^{4} \mathbf{P}\left[\Psi_{\mathrm{BT}}\left(\Re_{i}^{(\eta)}\right) \leq 3 y\right]
$$

by symmetry, (5.12) and the FKG inequality. Now by (5.8) and the definition of $\mu$, we have $\mu / 2 \geq \lambda_{i}^{y} / 32>(\eta-1) T_{i}$, so $2 T_{i}-\eta T_{i}+\mu>\eta T_{i}$. Hence, by Corollary 2.9 applied with $k=2$ (recalling that $\tilde{\mathfrak{R}}_{i}^{(\eta)}$ is landscape), we have

$$
\mathbf{P}\left[\Psi_{\mathrm{BT}}\left(\mathfrak{R}_{i}^{(\eta)}\right) \leq 3 y\right] \geq \mathbf{P}\left[\Psi_{\mathrm{LR}}\left(\tilde{\mathfrak{R}}_{i}^{(\eta)}\right) \leq y\right]^{3}-o(1) \geq p_{1}^{6} / 8-o(1),
$$

where the second inequality is by (5.15). Combining this last inequality with (5.16), we obtain

$$
\mathbf{P}\left[\Psi_{\mathrm{X} ;(\nu-\mu) / 2}\left(\tilde{\mathfrak{R}}_{i}^{(\eta)}\right) \leq 9 y\right] \geq \mathbf{P}\left[\Psi_{\mathrm{X} ; \nu / 2}\left(\tilde{\mathfrak{R}}_{i}^{(\eta)}\right) \leq 9 y\right] \geq p_{1}^{10} / 8-o(1),
$$

completing the proof of the lemma in this case, as long as $p_{2} \leq p_{1}^{10} / 8-o(1)$.

LEMMA 5.7. There is a $p_{3}$, depending only on $p_{1}$ and $p_{2}$, so that if $\gamma$ is sufficiently small compared to $p_{1}$ and $p_{2}$ then the following statement holds. Suppose that $y \geq w_{i}^{(\eta)}, \eta<\frac{256}{255}$ and $z \geq 0$ are such that either:

1. $z \geq w_{i-1}^{(\eta)}$ and $\lambda_{i}^{y} \leq \frac{7}{4} \lambda_{i-1}^{z}$ and $\eta-\frac{\lambda_{i-1}^{z}}{32 T_{i-1}}<1$ (in which the third inequality says that (5.8) holds at scale $i-1$ with weight $z)$, or

2. $\lambda_{i}^{y}=\eta T_{i} / 8$.

Then

$$
\mathbf{P}\left[\Psi_{\mathrm{LR}}\left(\left[0,5 T_{i} / 4\right) \times\left[0, \eta T_{i}\right)\right) \leq 55 y+11 z\right] \geq p_{3} .
$$

PROOF. If

$$
\mathbf{P}\left[\Psi_{\mathrm{LR}}\left(\left[0,2 T_{i}\right) \times\left[0, \eta T_{i}\right)\right) \leq 3 y\right] \geq p_{1},
$$


(i.e., if statement 1(a) from Lemma 5.4 holds) then there is nothing more to show as long as $p_{3} \leq p_{1}-o(1)$, since horizontally crossing a $T_{i} \times \eta T_{i}$ box implies horizontally crossing a $\frac{5}{4} T_{i} \times \eta T_{i}$ box. Similarly, if

$$
\mathbf{P}\left[\Psi_{\mathrm{LR}}\left(\left[0,2 T_{i-1}\right) \times\left[0, \eta T_{i-1}\right)\right) \leq 3 z\right] \geq p_{1},
$$

then we have (using (5.3))

$$
\begin{aligned}
& \mathbf{P}\left[\Psi_{\mathrm{LR}}\left(\left[0,5 T_{i} / 4\right) \times\left[0, \eta T_{i}\right)\right) \leq 4 z\right] \\
& \quad \geq \mathbf{P}\left[\Psi_{\mathrm{LR}}\left(\left[0,2 T_{i-1}\right) \times\left[0, \eta T_{i-1}\right) \leq 3 z\right]-o(1)\right. \\
& \quad \geq p_{1}-o(1),
\end{aligned}
$$

so there is nothing more to show as long as long as $p_{3} \leq p_{1}-o(1)$.

Thus we may henceforth assume that statement 1(b) from Lemma 5.4 holds for both $i$ (with weight $y=y$ ) and $i-1$ (with weight $y=z$ ). The rest of the proof is divided into two cases.

Case 1. If $\lambda_{i}^{y}=\eta T_{i} / 8$, then $\eta-\frac{1}{32 T_{i}} \lambda_{i}^{y}=\frac{255}{256} \eta<1$, so Lemma 5.6 implies that

$$
\mathbf{P}\left[\Psi_{\mathrm{X} ;\left(v_{i}^{y}-\mu_{i}^{y}\right) / 2}\left(\Re_{i}^{(\eta)}\right) \leq 9 y\right] \geq p_{2} .
$$

Since $v_{i}^{y}-\mu_{i}^{y}=2\left(\lambda_{i}^{y}-\mu_{i}^{y}\right) \geq \frac{7}{4} \lambda_{i}^{y}=\frac{7}{32} \eta T_{i}$ (recalling (5.9) and (5.10)), the intersection of six vertically translated copies of the event

$$
\left\{\Psi_{\mathrm{X} ;\left(v_{i}^{y}-\mu_{i}^{y}\right) / 2}\left(\mathfrak{R}_{i}^{(\eta)}\right) \leq 9 y\right\}
$$

contains a translate of the event

$$
\left\{\Psi_{\mathrm{BT}}\left(\left[0, T_{i}\right) \times\left[0, \frac{21}{16} \eta T_{i}\right)\right) \leq 54 y\right\}
$$

(as illustrated in Figure 6), and so also contains a translate of the event

$$
\left\{\Psi_{\mathrm{BT}}\left(\left[0, T_{i}\right) \times\left[0, \frac{5}{4} T_{i}\right)\right) \leq 54 y+7 z\right\} .
$$

So, by the FKG inequality and (2.5),

$$
\mathbf{P}\left[\Psi_{\mathrm{BT}}\left(\left[0, T_{i}\right) \times\left[0, \frac{5}{4} T_{i}\right)\right) \leq 55 y+8 z\right] \geq p_{2}^{6}-o(1) .
$$

This completes the proof of the lemma in the case when $\lambda_{i}^{y}=\eta T_{i} / 8$, as long as $p_{3} \leq p_{2}^{6}-o(1)$.

Case 2. Thus we can assume that $\lambda_{i}^{y}<\frac{\eta T_{i}}{8}$, so assumption 1 holds, which is to say that $z \geq w_{i-1}^{(\eta)}$ and $\lambda_{i}^{y} \leq \frac{7}{4} \lambda_{i-1}^{z}$ and (5.8) holds at scale $i-1$ with weight $z$. Now consider $\mathfrak{R}^{1}=\mathfrak{R}_{i}^{(\eta)}$ and $\mathfrak{R}^{2}=\mathfrak{R}_{i}^{(\eta)}+\left(T_{i}-T_{i-1}, 0\right)$, and

$$
\tilde{\mathfrak{R}}=\mathfrak{R}_{i-1}^{(\eta)}+\left(T_{i}-T_{i-1}, \frac{1}{2}\left(\eta T_{i}-\eta T_{i-1}+v_{i-1}-\mu_{i-1}\right)\right) .
$$




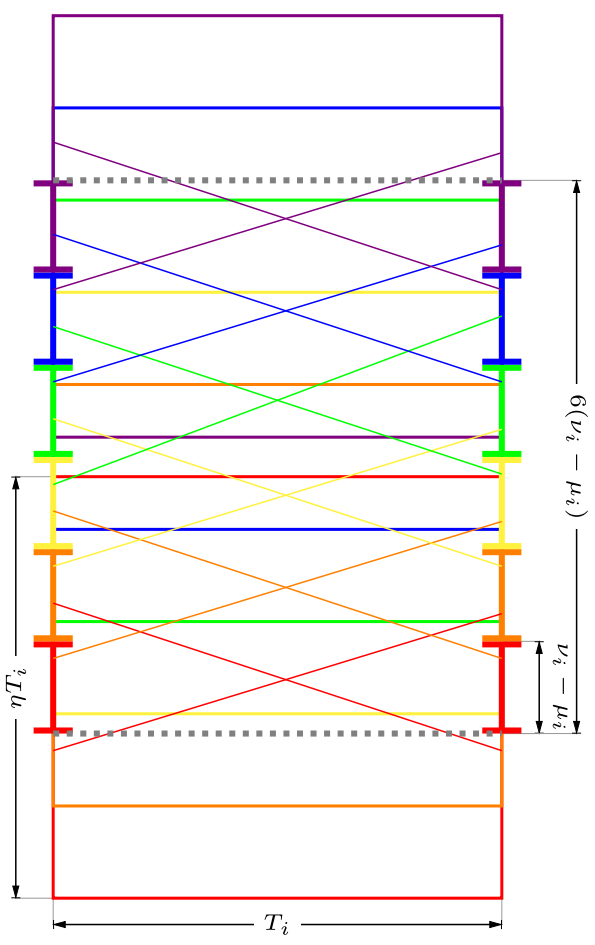

FIG. 6. A vertical crossing between the two dotted lines is obtained by combining the " $X$ " shapes, which must cross because their endpoints must straddle the interval of their color.

Note that, since

$$
\begin{aligned}
& \frac{\eta T_{i}-\eta T_{i-1}+v_{i-1}-\mu_{i-1}}{2}+\eta T_{i-1} \\
& \leq \frac{\eta T_{i}+\eta T_{i-1}}{2}+\lambda_{i-1} \\
& \leq \frac{\eta}{2}\left(T_{i}+T_{i-1}\right)+\frac{\eta}{8} T_{i-1} \\
& \leq \frac{\eta}{2}\left(T_{i}+\frac{3}{4} T_{i}\right)+\frac{3}{32} \eta T_{i}<\eta T_{i},
\end{aligned}
$$

we have $\tilde{\mathfrak{R}} \subset \mathfrak{R}^{1} \cap \mathfrak{R}^{2}$. Since $v_{i-1}^{z}-\mu_{i-1}^{z}=2\left(\lambda_{i-1}^{z}-\mu_{i-1}^{z}\right) \geq \frac{7}{4} \lambda_{i-1}^{z} \geq \lambda_{i}^{y}$, the event

$$
\left\{\Psi_{\mathrm{X} ;\left(v_{i-1}^{z}-\mu_{i-1}^{z}\right) / 2}(\tilde{\mathfrak{K}}) \leq 9 z\right\} \cap\left\{\Psi_{\mathrm{L}, 0, \lambda_{i}^{y}}\left(\mathfrak{R}^{1}\right) \leq y\right\} \cap\left\{\Psi_{0, \lambda_{i}^{y}, \mathrm{R}}\left(\mathfrak{R}^{2}\right) \leq y\right\}
$$

is contained in, up to coarse field error (i.e., the error bounded in (2.5)), the event

$$
\left\{\Psi_{\mathrm{LR}}\left(\left[0,2 T_{i}-T_{i-1}\right) \times\left[0, \eta T_{i}\right)\right) \leq 2 y+9 z\right\},
$$




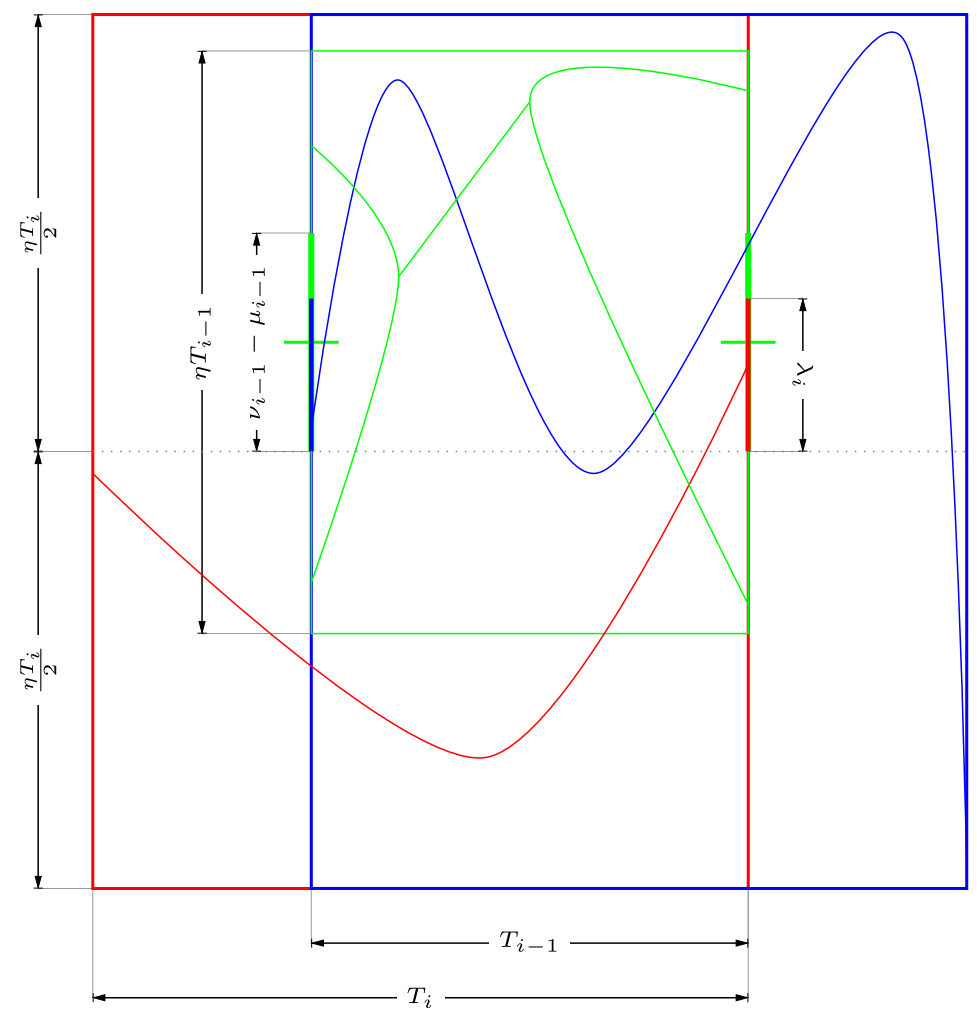

FIG. 7. The red and blue crossings are guaranteed to be joined by the green " $X$ " shape, since the red and blue crossings must remain within the red and blue boxes and end on the thick red and blue lines, respectively, while the green " $X$ " must have endpoints off of the thick green lines, which contain the thick red and blue lines.

since the crossings in the two larger rectangles must both intersect the " $\mathrm{X}$ " shape in the smaller rectangle, as they both must end on an interval that is contained in an interval that must be straddled by the endpoints of the " $\mathrm{X}$ " (see Figure 7). Hence, by the FKG inequality and (2.5), we have

$$
\begin{aligned}
& \mathbf{P}\left[\Psi_{\mathrm{LR}}\left(\left[0,2 T_{i}-T_{i-1}\right) \times\left[0, \eta T_{i}\right)\right) \leq 3 y+10 z\right] \\
& \quad \geq \mathbf{P}\left[\Psi_{\mathrm{X} ;\left(v_{i-1}^{z}-\mu_{i-1}^{z}\right) / 2}(\tilde{\mathfrak{R}}) \leq 9 z\right] \cdot \mathbf{P}\left[\Psi_{\mathrm{L}, 0, \lambda_{i}^{y}}\left(\mathfrak{R}^{1}\right) \leq y\right]^{2}-o(1) \\
& \quad \geq p_{1}^{2} \mathbf{P}\left[\Psi_{\mathrm{X} ;\left(v_{i-1}^{z}-\mu_{i-1}^{z}\right) / 2}(\tilde{\mathfrak{R}}) \leq 9 z\right]-o(1) .
\end{aligned}
$$

Now by Lemma 5.6, recalling our assumption that (5.8) holds at scale $i-1$ with weight $z$, if $\gamma$ is sufficiently small compared to $p_{2}$, we have

$$
\mathbf{P}\left[\Psi_{\mathrm{X} ;\left(v_{i-1}^{z}-\mu_{i-1}^{z}\right) / 2}(\tilde{\mathfrak{R}}) \leq 9 z\right] \geq p_{2}
$$


So

$$
\begin{aligned}
& \mathbf{P}\left[\Psi_{\mathrm{LR}}\left(\left[0,5 T_{i} / 4\right) \times\left[0, \eta T_{i}\right)\right) \leq 4 y+11 z\right] \\
& \quad \geq \mathbf{P}\left[\Psi_{\mathrm{LR}}\left(\left[0,2 T_{i}-T_{i-1}\right) \times\left[0, \eta T_{i}\right)\right) \leq 3 y+10 z\right]-o(1) \\
& \quad \geq p_{1}^{2} p_{2} / 2-o(1),
\end{aligned}
$$

completing the proof of the lemma in the case when $\lambda_{i}^{y}<\eta T_{i} / 8$, as long as $p_{3} \leq$ $\frac{1}{2} p_{1}^{2} p_{2}-o(1)$.

LEMMA 5.8. There are constants $c_{1}$ and $p_{4}$, depending only on $p_{3}$, so that the following holds. Let $j \geq i+8$. Suppose that $\eta \leq 9 / 8, \lambda_{j}^{w_{j}^{(\eta)}} \leq \eta T_{i}, \gamma$ is sufficiently small compared to $p_{3}$, and

$$
\mathbf{P}\left[\Psi_{\mathrm{LR}}\left(\left[0,5 T_{i} / 4\right) \times\left[0, \eta T_{i}\right)\right) \leq y\right] \geq p_{3} .
$$

Then

$$
\mathbf{P}\left[\Psi_{\mathrm{LR}}\left(\left[0,2 T_{j}\right) \times\left[0, \eta T_{j}\right)\right) \leq 2 w_{j}^{(\eta)}+c_{1} y\right] \geq p_{4}-o_{j-i}(1) .
$$

ProOF. Since $j \geq i+8$, we have $T_{j} \geq 16 T_{i}$, so $\lambda_{j}^{y} \leq \eta T_{i}<\eta T_{j} / 8$, so by statement 2 of Lemma 5.4, we have

$$
\mathbf{P}\left[\Psi_{\mathrm{L}, 0, \lambda_{j} w_{j}^{(\eta)}}\left(\Re_{j}^{(\eta)}\right) \leq w_{i}^{(\eta)}\right] \geq p_{0} / 4
$$

and

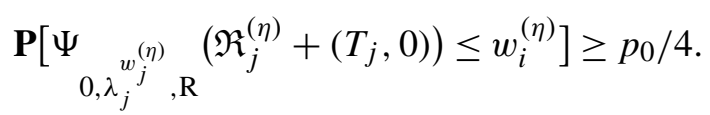

Now we can build an annulus $\mathfrak{L}$, whose inner square has width $\eta T_{i}$ and whose outer square has width $3 \eta T_{i}$, inside $\mathfrak{R}_{j}^{(\eta)} \cup\left(\mathfrak{R}_{j}^{(\eta)}+\left(T_{j}, 0\right)\right)$, such that $\mathfrak{L}$ surrounds $T_{j} \times\left[\eta T_{j} / 2, \eta T_{j} / 2+\lambda_{j}^{w_{j}^{(\eta)}}\right]$.

By (5.17), our upper bound on $\eta$ and Corollary 2.9, we have constants $c_{2}$ and $p_{5}$, depending only on $p_{3}$ and the facts that $\eta$ is a constant amount less than $5 / 4$ and that $\gamma$ is sufficiently small, so that

$$
\mathbf{P}\left[\Psi_{\mathrm{LR}}\left(\left[0,3 \eta T_{i}\right) \times\left[0, \eta T_{i}\right)\right) \leq c_{2} y\right] \geq p_{5} .
$$

Let $E$ denote the event that there is a circuit of $Y_{\mathfrak{R}_{j}^{(\eta)} \cup\left(\mathfrak{R}_{j}^{(\eta)}+\left(T_{j}, 0\right)\right)}$-weight at most $5 c_{2} y$ around $\mathfrak{L}$, and let $E_{1}, E_{2}, E_{3}, E_{4}$ denote rotated and translated copies of $\left\{\Psi_{\mathrm{LR}}\left(\left[0,3 \eta T_{i}\right) \times\left[0, \eta T_{i}\right)\right) \leq c_{2} y\right\}$ whose intersection, up to coarse field error, contains $E$. Now $\mathbf{P}\left[E_{\alpha}\right]=\mathbf{P}\left[\Psi_{\mathrm{LR}}\left(\left[0,3 \eta T_{i}\right) \times\left[0, \eta T_{i}\right)\right) \leq c_{2} y\right] \geq p_{5}$, and so, by the FKG inequality and (2.5), we have

$$
\mathbf{P}[E] \geq p_{5}^{4}-o_{j-i}(1) .
$$




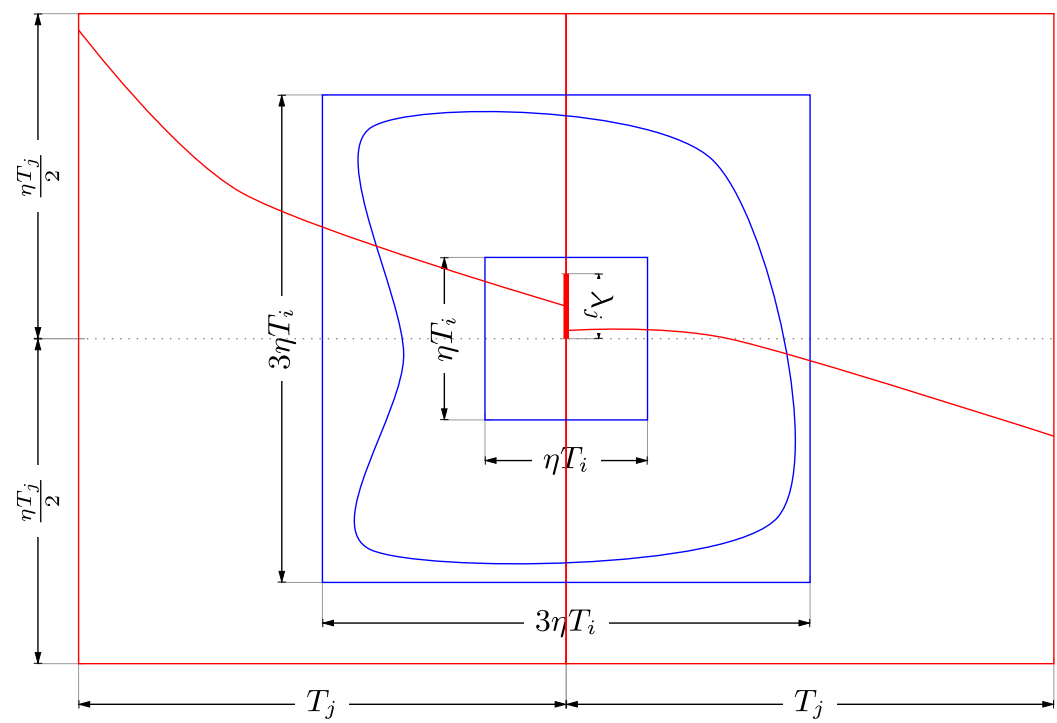

FIG. 8. Geometric construction in the proof of Lemma 5.8. The two red crossings are connected by the blue circuit. (Again we omit the weight subscripts.)

Since, up to coarse field error, we have

$$
\begin{aligned}
& \left\{\Psi_{\mathrm{LR}}\left(\left[0,2 T_{j}\right) \times\left[0, \eta T_{j}\right)\right) \leq 2 w_{j}^{(\eta)}+5 c_{2} y\right\} \\
& \supset E \cap\left\{\underset{\mathrm{L}, 0, \lambda_{j}^{w_{j}^{(\eta)}}}{ }\left(\Re_{j}^{(\eta)}\right) \geq w_{i}^{(\eta)}\right\} \cap \underset{\left.0, \lambda_{j}^{w_{j}^{(\eta)}} \underset{, \mathrm{R}}{\Psi}\left(\Re_{j}^{(\eta)}+\left(T_{j}, 0\right)\right) \leq w_{i}^{(\eta)}\right\}}{\{}
\end{aligned}
$$

(see Figure 8), the FKG inequality, along with (5.18), (5.19) and (5.20), tells us that

$$
\mathbf{P}\left[\Psi_{\mathrm{LR}}\left(\left[0,2 T_{j}\right) \times\left[0, \eta T_{j}\right)\right) \leq 3 w_{j}^{(\eta)}+5 c_{2} y\right] \geq\left(p_{0} / 4\right)^{2}\left(p_{5} / 2\right)^{4}-o_{j-i}(1),
$$

establishing the lemma with $c_{1}=5 c_{2}$ and $p_{4}=\left(p_{0} / 4\right)^{2}\left(p_{5} / 2\right)^{4}$.

5.3. Multiscale analysis. We now turn to the multiscale analysis involved in the proof of Theorem 5.1.

LEMMA 5.9. Let $c_{3}$ be so large that

$$
\left(1-p_{4}^{15}\right)^{\left\lfloor c_{3} / 4\right\rfloor} \leq p_{0} / 8 .
$$

Suppose that $\eta \leq \frac{256}{255}$ and that (5.17) holds for $i$ and $y$. For any $\Delta \geq 6$, there is $a$ $j \in\left[i+\Delta, i+\Delta+c_{3}\right]$ so that, if $\gamma$ is sufficiently small relative to $\Delta$, then

$$
\lambda_{j}^{21 W_{j}^{(\eta)}+10 c_{1} y} \geq \eta T_{i}
$$


PROOF. Let $\tilde{j}=i+\Delta+c_{3}$. Suppose for the sake of contradiction that, for all $i+\Delta<j \leq \tilde{j}$, we have $\lambda_{j}^{w_{j}^{(\eta)}}<\eta T_{i}$, and moreover that we have

$$
\lambda_{\tilde{j}}^{w_{\tilde{j}}^{(\eta)}+2\left(10 W_{\tilde{j}}+5 c_{1} y\right)}<\eta T_{i} .
$$

Then Lemma 5.8 implies that

$$
\mathbf{P}\left[\Psi_{\mathrm{LR}}\left(\left[0,2 T_{j}\right) \times\left[0, \eta T_{j}\right)\right) \leq 2 w_{j}^{(\eta)}+c_{1} y\right] \geq p_{4}-o_{\Delta}(1)
$$

for each $i+\Delta<j \leq \tilde{j}$. By Corollary 2.9, this yields

$$
\mathbf{P}\left[\Psi_{\mathrm{LR}}\left(\left[0,3 \eta T_{j}\right) \times\left[0, \eta T_{j}\right)\right) \leq 10 w_{j}^{(\eta)}+5 c_{1} y\right] \geq p_{4}^{5}-o_{\Delta}(1) .
$$

Let

$$
\begin{aligned}
& J_{1}=\left\{\Psi_{\mathrm{L}, 0, \eta T_{i}}\left(\Re_{\tilde{j}}^{(\eta)}\right) \leq w_{\tilde{j}}^{(\eta)}\right\} \text { and } \\
& J_{2}=\left\{\Psi_{\mathrm{L}, \eta T_{i}, \eta T_{\tilde{j}} / 2}\left(\Re_{\tilde{j}}^{(\eta)}\right) \leq w_{\tilde{j}}^{(\eta)}+2\left(10 W_{\tilde{j}}+5 c_{1} y\right)\right\} .
\end{aligned}
$$

Then (5.23) and Lemma 5.4(2) imply that we have

$$
\mathbf{P}\left[J_{1}\right]-\mathbf{P}\left[J_{2}\right] \geq p_{0} / 4 \text {. }
$$

Let $E$ be the event that there is a path in $\mathfrak{R}_{\tilde{j}}^{(\eta)}$ of weight at most $2\left(10 W_{\tilde{j}}+5 c_{1} y\right)$, from $\left\{T_{\tilde{j}}-1\right\} \times\left[\eta T_{\tilde{j}} / 2+\eta T_{i}, \eta T_{\tilde{j}}\right]$ to $\left\{T_{j}-1\right\} \times\left[0, \eta T_{j} / 2\right]$. Note that $J_{1} \cap E \subset J_{2}$, so

$$
\mathbf{P}\left[J_{2}\right] \geq \mathbf{P}\left[J_{1} \cap E\right] \geq \mathbf{P}\left[J_{1}\right] \mathbf{P}[E]
$$

by the FKG inequality. Combining (5.25) and (5.26), we get that

$$
\mathbf{P}\left[E^{c}\right] \geq \mathbf{P}\left[J_{1}\right] \mathbf{P}\left[E^{c}\right] \geq p_{0} / 4 .
$$

For each $i+\Delta \leq j<\tilde{j}$ such that $j \in 4 \mathbf{Z}$, let $E_{1}^{j}, E_{2}^{j}, E_{3}^{j}$ be the events that there are hard crossings — of weight at most $10 w_{j}^{(\eta)}+5 c_{1} y$-in, respectively, three rectangles of shorter side-length $\eta T_{j}$ and longer side-length $3 \eta T_{j}$, that together form a "C" shape connecting $\left\{T_{\tilde{j}}-1\right\} \times\left[\eta T_{\tilde{j}} / 2+\eta T_{i}, \eta T_{\tilde{j}}\right)$ to $\left\{T_{\tilde{j}}-1\right\} \times\left[0, \eta T_{j} / 2\right)$, and which moreover are chosen so that the blow-ups of the rectangles only intersect other rectangles corresponding to the same $j$. The setup is illustrated in Figure 9.

By (5.24), we have

$$
\mathbf{P}\left[E_{\alpha}^{j}\right] \geq p_{4}^{5}-o_{\Delta}(1) .
$$

Let $\tilde{E}_{1}^{j}, \tilde{E}_{2}^{j}, \tilde{E}_{3}^{j}$ be defined in the same way as $E_{1}^{j}, E_{2}^{j}, E_{3}^{j}$, except with the requirement that the $Y_{\mathfrak{R}_{\tilde{j}}^{(\eta)}}$-weight of the paths be at most $2\left(10 w_{j}^{(\eta)}+5 c_{1} y\right)$, rather than 


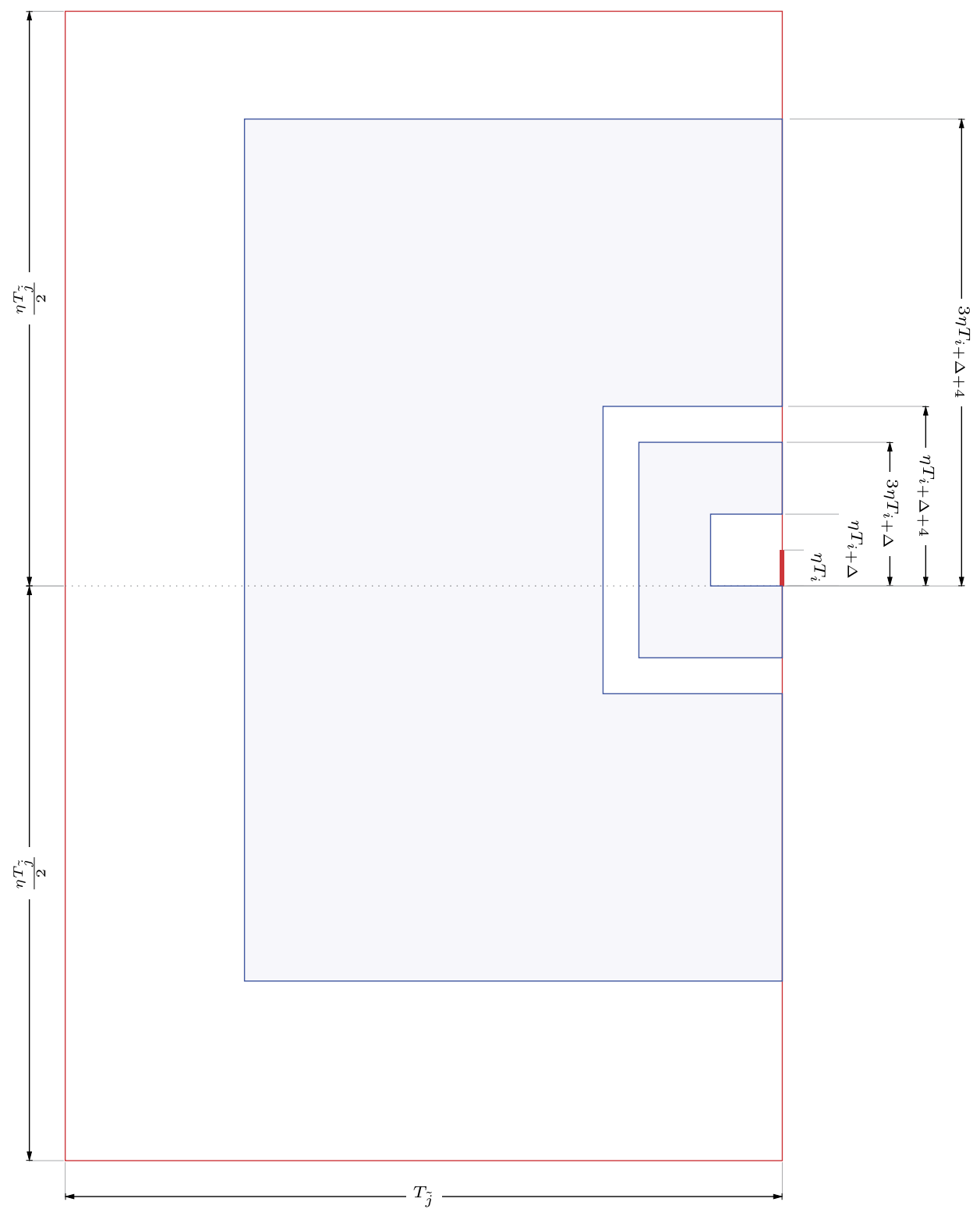

FIG. 9. Geometric construction in the proof of Lemma 5.9. (In reality there would be many more half-annuli!)

that the weight of the paths with respect to the GFF in their own rectangles be at most $10 w_{j}^{(\eta)}+5 c_{1} y$.

For each $j$, we have that $\tilde{E}_{1}^{j} \cap \tilde{E}_{2}^{j} \cap \tilde{E}_{3}^{j} \subset E$. Let $Z=\max _{\alpha, j, x} W_{\alpha}^{j}(x)$, where $W_{\alpha}^{j}$ is the coarse field correction term for the rectangle in $E_{\alpha}^{j}$ as in (2.5). Now 
note that $\tilde{E}_{1}^{j} \cap \tilde{E}_{2}^{j} \cap \tilde{E}_{3}^{j} \supset E_{1}^{j} \cap E_{2}^{j} \cap E_{3}^{j} \cap\{Z \leq 2\}$, so we can compute, using the independence of the fine fields,

$$
\begin{aligned}
\mathbf{P}\left[E^{c}\right] & \leq \mathbf{P}\left[\bigcap_{i+\Delta \leq j<\tilde{j}}\left(\tilde{E}_{1}^{j} \cap \tilde{E}_{2}^{j} \cap \tilde{E}_{3}^{j}\right)^{c}\right] \\
& \leq \mathbf{P}\left[\bigcap_{\substack{i+\Delta \leq j<\tilde{j} \\
j \in 4 \mathbf{Z}}}\left(\left(E_{1}^{j} \cap E_{2}^{j} \cap E_{3}^{j}\right)^{c} \cup\{Z>2\}\right)\right] \\
& \leq o_{\Delta}(1)+\prod_{i+\Delta \leq j<\tilde{j}}\left(1-\mathbf{P}\left[E_{1}^{j} \cap E_{2}^{j} \cap E_{3}^{j}\right]\right) \\
& \leq \prod_{j \in 4 \mathbf{Z}}\left(1-\mathbf{P}\left[E_{1}^{j}\right]^{3}\right)+o_{\Delta}(1) \\
& \leq\left(1-p_{4}^{15}\right)^{\left\lfloor c_{3} / 4\right\rfloor}+o_{\Delta}(1) .
\end{aligned}
$$

Now if $\gamma$ is small enough (relative to $\Delta$ ), then combined with (5.21) this implies that $\mathbf{P}\left[E^{c}\right]<p_{0} / 4$, contradicting (5.27). So either, for some $i+\Delta<j \leq \tilde{j}$, we have $\lambda_{j}^{w_{j}^{(\eta)}} \geq \eta T_{i}$, or else we have $\lambda_{\tilde{j}}^{w_{\tilde{j}}^{(\eta)}+2\left(10 W_{\tilde{j}}^{(\eta)}+5 c_{1} y\right)} \geq \eta T_{i}$, implying (5.22) in any case.

LEMMA 5.10. Write $f(k)=\lambda_{k}^{y_{k}}$ for some sequence $y_{k} \geq w_{k}^{(\eta)}$. Suppose that $f\left(k_{0}\right) \geq a \eta T_{k_{0}}$. Then if $c$ is so large that $\left(\frac{7}{4}\right)^{c} \frac{1}{\sqrt{2}^{c-1}}>\frac{1}{a}$, then there is $a$ $k \in\left(k_{0}, k_{0}+c\right]$ so that $f(k) \leq \frac{7}{4} f(k-1)$ and $f(k-1) \geq a \eta T_{k-1}$.

PROOF. If $f(k) \geq \frac{7}{4} f(k-1)$ for all $k_{0}<k \leq k_{0}+c$, then we have (using (5.4))

$$
\frac{1}{2} \eta T_{k_{0}+c} \geq f\left(k_{0}+c\right) \geq(7 / 4)^{c} a \eta T_{k_{0}} \geq(7 / 4)^{c} a \eta \frac{1}{\sqrt{2}^{c+1}} T_{k_{0}+c},
$$

contradicting our assumption on $c$. Therefore, there is some $k \in\left(k_{0}, k_{0}+c\right]$ so that $f(k) \leq \frac{7}{4} f(k-1)$. Moreover, if we choose the first such $k$, then we have

$$
\frac{f(j)}{T_{j}} \geq \frac{7}{4} \cdot \frac{f(j-1)}{T_{j}} \geq \frac{7}{4} \cdot \frac{f(j-1)}{3 T_{j-1} / 2} \geq \frac{f(j-1)}{T_{j-1}}
$$

for all $k_{0}<j<k$, so by induction we have $f(k-1) \geq a \eta T_{k-1}$. 
LEMMA 5.11. Let $c_{5}=\max \left\{1386,660 c_{1}\right\}$. Fix $\Delta \geq 6$ and suppose that

$$
1<\eta \leq 1+\frac{1}{32 \sqrt{2}^{\Delta+c_{3}+1}} .
$$

Then if $\gamma$ is sufficiently small relative to $\Delta$, then there is a $\chi(\Delta) \geq \Delta$ so that if (5.17) holds for $i$ and $y$, then there is a $k \in[i+\Delta, i+\chi(\Delta)]$ so that (5.17) holds for $i=k$ and $y=c_{5}\left(W_{k}^{(\eta)}+y\right)$.

Proof. By Lemma 5.9, there is a $j \in\left[i+\Delta, i+\Delta+c_{3}\right]$ so that (using (5.4)) we have $\lambda_{j}^{21 W_{j}^{(\eta)}+10 c_{1} y} \geq \eta T_{i} \geq \frac{\eta T_{j}}{\sqrt{2}^{\Delta+c_{3}+1}}$. Let $\xi(\Delta)$ be so large that $\frac{(7 / 4)^{\xi(\Delta)}}{\sqrt{2}^{\xi(\Delta)-1}}>$ $\sqrt{2}^{\Delta+c_{3}+1}$. Then if we put $f(k)=\lambda_{k}^{11 e W_{k}^{(n)}+5 e c_{1} y}$ and let $\chi(\Delta)=\xi(\Delta)+c_{3}$, by Lemma 5.10 there is some $k \in(j, j+\xi(\Delta)] \subset\left[i+\Delta, i+\Delta+c_{3}+\xi(\Delta)\right]=$ $[i+\Delta, i+\chi(\Delta)]$ so that

$$
\lambda_{k}^{21 W_{k}^{(n)}+10 c_{1} y} \leq \frac{7}{4} \lambda_{k-1}^{21 W_{k-1}^{(\eta)}+10 c_{1} y}
$$

and

$$
\frac{1}{32} \lambda_{k-1}^{21 W_{k-1}^{(\eta)}+10 c_{1} y} \geq \frac{\eta T_{k-1}}{32 \sqrt{2}^{\Delta+c_{3}+1}} \geq \frac{T_{k-1}}{32 \sqrt{2}^{\Delta+c_{3}+1}}>(\eta-1) T_{k-1},
$$

with the last inequality by (5.28). Thus the hypotheses of Lemma 5.7 hold with $i=k, y=21 W_{k}^{(\eta)}+10 c_{1} y$ and $z=21 W_{k-1}^{(\eta)}+10 c_{1} y$ (where the left-hand sides are in the notation of the statement of Lemma 5.7 and the right-hand sides are in the notation of the present proof). This means that

$$
\begin{aligned}
& \mathbf{P}\left[\Psi_{\mathrm{LR}}\left(\left[0, \frac{5 T_{k}}{4}\right) \times\left[0, \eta T_{k}\right)\right) \geq 55\left(21 W_{k}^{(\eta)}+10 c_{1} y\right)+11\left(21 W_{k-1}^{(\eta)}+10 c_{1} y\right)\right] \\
& \quad \geq p_{3},
\end{aligned}
$$

which is to say that (5.17) holds with $y=c_{5}\left(W_{k}^{(\eta)}+y\right)$ (where again the left-hand side is in the notation of (5.17) and the right-hand side is in the present notation).

LEMMA 5.12. Fix $\Delta \geq 6$ and suppose that $\eta-1 \leq 2^{-\left(\Delta+c_{3}+7\right)}$. Then there is an increasing sequence $1=i_{1}, i_{2}, i_{3}, \ldots$ so that

$$
i_{r+1} \in\left[i_{r}+\Delta, i_{r}+\chi(\Delta)\right] \text {, }
$$

and, for each $r$, (5.17) holds for $i=i_{r}$ and

$$
y=\sum_{s=1}^{r} c_{5}^{r+1-s}\left(W_{i_{s}}^{(\eta)} \vee y_{1}^{*}\right),
$$

where we recall that $y_{1}^{*}$ is defined to be the quantity satisfying (5.6). 
PROOF. According to (5.6), we have $\lambda_{1}^{y_{1}^{*}}=\eta T_{1} / 8$. This means that Lemma 5.7 applies, so (5.17) holds for $i=1$ and $y=42 y_{1}^{*}$. In other words, if we put $i_{1}=1$, then the conclusion of the lemma holds for $r=1$.

Now we claim that once we have chosen a suitable $i_{r}$, then we can also choose a suitable $i_{r+1}$. Indeed, if (5.17) holds for $i=i_{r}$ and

$$
y=\sum_{s=1}^{r} c_{5}^{r+1-s}\left(W_{i_{s}}^{(\eta)} \vee y_{1}^{*}\right)
$$

then Lemma 5.11 implies that there is an $i_{r+1} \in\left[i_{r}+\Delta, i_{r}+\chi(\Delta)\right]$ so that (5.17) holds for $i=i_{r+1}$ and

$$
y=c_{5}\left(W_{i_{r+1}}+\sum_{s=1}^{r} c_{5}^{r+1-s}\left(W_{i_{s}}^{(\eta)} \vee y_{1}^{*}\right)\right) \leq \sum_{s=1}^{r+1} c_{5}^{r+2-s}\left(W_{i_{s}}^{(\eta)} \vee y_{1}^{*}\right),
$$

hence for $y=\sum_{s=1}^{r+1} c_{5}^{r+2-s}\left(W_{i_{s}}^{(\eta)} \vee y_{1}^{*}\right)$ as well. This completes the inductive step of the proof of the lemma.

The next lemma uses the fact that our desired results at a given scale imply the same results at constant multiples of the scale to extend Lemma 5.12 to all scales, and also to better-shaped boxes.

LEMMA 5.13. Fix $\Delta \geq 6$ and suppose that $\eta-1 \leq 2^{-\left(\Delta+c_{3}+7\right)}$. We have constants $p(\Delta)$ and $C(\Delta)$ so that for each $i \geq 1$, we have

$$
\Theta_{\text {hard }}\left(\Re_{i}\right)[p(\Delta)] \leq C(\Delta) \sum_{j=0}^{\lfloor i / \Delta\rfloor} c_{5}^{j}\left(W_{i-1-j \Delta}^{(\eta)} \vee y_{1}^{*}\right)
$$

PRoOF. By Lemma 5.12, there is an $i_{r}$ so that $i-1-\chi(\Delta) \leq i_{r} \leq i-1$ and

$$
\mathbf{P}\left[\Psi_{\mathrm{LR}}\left(\left[0,5 T_{i_{r}} / 4\right) \times\left[0, \eta T_{i_{r}}\right)\right) \leq y_{r}\right] \geq p_{3},
$$

where

$$
y_{r}=\sum_{\alpha=1}^{r} c_{5}^{r+1-\alpha}\left(W_{i_{\alpha}}^{(\eta)} \vee y_{1}^{*}\right) .
$$

Note that (5.29) implies that, for each $\alpha$, we have $i_{q} \leq i_{r}-(r-\alpha) \Delta$. This means that

$$
\begin{aligned}
y_{r} \leq \sum_{\alpha=1}^{r} c_{5}^{r+1-\alpha}\left(W_{i_{r}-(r-\alpha) \Delta}^{(\eta)} \vee y_{1}^{*}\right) & =\sum_{j=0}^{r-1} c_{5}^{j+1}\left(W_{i_{r}-j \Delta}^{(\eta)} \vee y_{1}^{*}\right) \\
& \leq \sum_{j=0}^{r-1} c_{5}^{j+1}\left(W_{i-1-j \Delta}^{(\eta)} \vee y_{1}^{*}\right) .
\end{aligned}
$$

Now Corollary 2.9 and (2.5) imply the desired result. 
We are finally ready to prove our RSW result.

Proof of TheOrem 5.1. Choose $\kappa$ so large that

$$
\begin{aligned}
a_{\mathrm{pl}}^{\kappa} & <\frac{1}{4 c_{5}} \text { and } \\
c_{5}^{\frac{1}{2 \kappa}} & <1 / a_{\mathrm{pl}} .
\end{aligned}
$$

Put $\Delta=\lceil 2 \kappa\rceil$ and apply Lemma 5.13. Fix $\eta$ as in the statement of that lemma; then we have

$$
\begin{aligned}
& \Theta_{\text {hard }}\left(\Re_{i}\right)[p(\Delta)] \\
& \quad \leq C(\Delta) \sum_{j=0}^{\left\lfloor\frac{i}{2 \kappa}\right\rfloor} c_{5}^{j}\left(W_{i-1-j \Delta}^{(\eta)} \vee y_{1}^{*}\right) \\
& \quad \leq C_{\text {str }}(\eta) C(\Delta) \sum_{j=0}^{\left\lfloor\frac{i}{2 \kappa}\right\rfloor} c_{5}^{j}\left(\max _{\alpha \leq i-1-j \Delta} \Theta_{\text {easy }}\left(\Re_{\alpha}\right)\left[p_{\text {str }}(\eta)\right] \vee y_{1}^{*}\right) \\
& \quad \leq C_{\text {str }}(\eta) C(\Delta) \sum_{j=0}^{\left\lfloor\frac{i}{2 \kappa}\right\rfloor} c_{5}^{j} \max _{\alpha \leq i-1-j \Delta} \Theta_{\text {easy }}\left(\Re_{\alpha}\right)\left[p_{\text {str }}(\eta)\right]+C_{3} y_{1}^{*} \sum_{j=0}^{\left\lfloor\frac{i}{2 \kappa}\right\rfloor} c_{5}^{j},
\end{aligned}
$$

with the second inequality by Corollary 5.3 .

Our goal is to relate the sums in (5.34) to a quantile of an easy crossing of $\mathfrak{R}_{i}$, and our primary tool will be the a priori power-law lower bound of sufficiently small crossing quantiles given in Proposition 4.11. However, Proposition 4.11 only relates very small quantiles, and the quantiles in (5.34) (coming from Corollary 5.3) are very large. This is the reason for the assumption (5.2): by applying (3.2), this assumption lets us relate very small and very large quantiles, assuming $\delta$ is chosen sufficiently small.

Now we put this plan into action. For each $j$, we have

$$
\max _{\alpha \leq i-1-j \Delta} \Theta_{\text {easy }}\left(\Re_{\alpha}\right)\left[p_{\text {str }}(\eta)\right] \leq C \max _{\alpha \leq i-1-j \Delta} \Theta_{\text {easy }}\left(\Re_{\alpha}\right)\left[p_{\text {pl }}\right]
$$

(with $p_{\mathrm{pl}}$ as in Proposition 4.11) by (5.2) and (3.2), choosing $\delta$ small enough (depending on $p_{\text {str }}$ and $p_{\mathrm{pl}}$ ) so that the necessary assumptions hold. But then by Proposition 4.11, we have

$$
\begin{aligned}
& \max _{\alpha \leq i-1-j \Delta} \Theta_{\text {easy }}\left(\Re_{\alpha}\right)\left[p_{\text {str }}(\eta)\right] \\
& \quad \leq C C_{\mathrm{pl}} a_{\mathrm{pl}}^{j \Delta+1} \cdot \Theta_{\text {easy }}\left(\Re_{i}\right)\left[q_{\mathrm{pl}}\right] .
\end{aligned}
$$


This gives us

$$
\begin{gathered}
\sum_{j=0}^{\left\lfloor\frac{i}{2 \kappa}\right\rfloor} c_{5}^{j} \max _{\alpha \leq i-1-j \Delta} \Theta_{\text {easy }}\left(\Re_{\alpha}\right)\left[p_{\text {str }}(\eta)\right] \\
\leq C C_{\mathrm{pl}} \Theta_{\text {easy }}\left(\Re_{i}\right)[q] \sum_{j=0}^{\left\lfloor\frac{i}{2 \kappa}\right\rfloor} c_{5}^{j} a_{\mathrm{pl}}^{j \Delta+1} \\
\leq C^{\prime} \Theta_{\text {easy }}\left(\Re_{i}\right)[q],
\end{gathered}
$$

where in the last inequality we use (5.32). Moreover, we have

$$
\sum_{j=0}^{\left\lfloor\frac{i}{2 \kappa}\right\rfloor} c_{5}^{j} \leq \frac{c_{5}^{\frac{i}{2 \kappa}+1}-1}{c_{5}-1} \leq C^{\prime \prime} \Theta_{\text {easy }}\left(\Re_{i}\right)[q],
$$

with the last inequality by (5.33) and Proposition 4.11 .

Now choose

$$
p_{\mathrm{RSW}} \leq \min \left\{p(\Delta), p_{\mathrm{pl}},\left(32 \cdot d_{\mathrm{p}}^{2}\right)^{-2}\right\} .
$$

Plugging (5.35) and (5.36) into (5.34), we obtain that (5.1) holds and that

$$
\begin{aligned}
\Theta_{\text {hard }}\left(\Re_{S}\right)\left[p_{\mathrm{RSW}}\right] & \leq \Theta_{\text {hard }}\left(\Re_{S}\right)[p(\Delta)] \\
& \leq C^{\prime \prime \prime} \Theta_{\text {easy }}\left(\Re_{S}\right)[q] \\
& \leq C^{\prime \prime \prime} \Theta_{\text {easy }}\left(\Re_{S}\right)\left[p_{\text {RSW }}\right] .
\end{aligned}
$$

Here the second inequality is by (3.2) and (5.2) as long as $\delta$ is sufficiently small compared to $p_{\mathrm{RSW}}$.

6. Upper bounds on FPP distance and geodesic length. In this section we derive upper bounds on the crossing distance, geodesic length and box diameter.

6.1. Crossing distance upper bound. We want to derive a right-tail bound on the crossing distance in terms of the hard crossing distance at a smaller scale. We show this by showing that hard crossings from smaller scales can be glued together to get a crossing at a larger scale, and that there are many nearly independent opportunities for this to happen, so we get good control on the right tail of the crossing distance.

Let $\mathfrak{R}=[0, K S) \times[0, L S)$. Let $\mathfrak{C}=[0, S)^{2}$ and $\mathfrak{A}=[0, S) \times[0,2 S)$. Index the dyadic subboxes of $\mathfrak{R}$ having side length $S$ by row and column according to the following layout:

$$
\begin{array}{ccc}
\mathfrak{C}_{11} & \cdots & \mathfrak{C}_{1 L} \\
\vdots & \ddots & \vdots \\
\mathfrak{C}_{K 1} & \cdots & \mathfrak{C}_{K L}
\end{array}
$$


Proposition 6.1. If $u>0$, we have

$$
\mathbf{P}\left[\Psi_{\mathrm{LR}}(\mathfrak{R}) \geq 2 u K \mathbf{E} \Psi_{\text {hard }}(\mathfrak{A})\right] \leq u^{-L / 3}+o_{K, L}(1) .
$$

Moreover, if $u \geq u_{0}$ (defined in (2.6)), then we have

$$
\mathbf{P}\left[\Psi_{\mathrm{LR}}(\mathfrak{R}) \geq 2 u K \mathbf{E} \Psi_{\text {hard }}(\mathfrak{A})\right] \leq u^{-L / 4}+\exp \left(-\omega(1) \cdot \frac{(\log u)^{2}}{\log (K \vee L)}\right) .
$$

Finally, as long as $L \geq 10$ we have

$$
\mathbf{E} \Psi_{\mathrm{LR}}(\mathfrak{R})^{3} \leq O_{K, L}(1)\left(\mathbf{E} \Psi_{\text {hard }}(\mathfrak{A})^{2}\right)^{3 / 2} .
$$

ProOF. For each $0 \leq j \leq L-1$ such that $3 \mid j$, let $\Psi_{j}=\Psi_{\mathrm{LR}}((0, j S)+$ $[0, K S) \times[0, S))$. [Note here that $(0, j S)$ is a point in $\mathbf{Z}^{2}$, not an open interval.] Then for each $j$, by (2.5) and the strategy illustrated in Figure 2 we have

$$
\Psi_{j} \leq \sum_{i=1}^{K-1} \Psi_{\text {hard }}\left(\mathfrak{C}_{j, i} \cup \mathfrak{C}_{j, i+1}\right)+\sum_{i=2}^{K-1} \Psi_{\text {hard }}\left(\mathfrak{C}_{j, i} \cup \mathfrak{C}_{j \pm 1, i}\right) .
$$

Thus we have

$$
\mathbf{E} \Psi_{j} \leq\left(1+o_{K, L}(1)\right)(2 K-3) \mathbf{E} \Psi_{\text {hard }}(\mathfrak{A}) \leq 2 K \mathbf{E} \Psi_{\text {hard }}(\mathfrak{A})
$$

as long as $\gamma$ is sufficiently small compared to $K$ and $L$. Applying Markov's inequality gives us

$$
\mathbf{P}\left[\Psi_{j} \geq 2 u K \mathbf{E} \Psi_{\text {hard }}(\mathfrak{A})\right] \leq 1 / u .
$$

Since up to coarse field error we have $\Psi_{\mathrm{LR}}(\mathfrak{R}) \leq \min _{j} \Psi_{j}$, and the set $\left\{\Psi_{j} \mid 0 \leq\right.$ $j \leq L-1$ and 3 divides $j\}$ is independent, we have (6.1) by (2.5) and (6.2) by (2.6) and the assumption that $u \geq u_{0}$. Finally, the Cauchy-Schwarz inequality and Lemma 6.2 below give us

$$
\mathbf{E} \Psi_{\mathrm{LR}}(\mathfrak{R})^{3} \leq O_{K, L}(1) \cdot\left(\mathbf{E} \Psi_{j}^{2}\right)^{3 / 2} \leq O_{K, L}(1)\left(\mathbf{E} \Psi_{\text {hard }}(\mathfrak{A})^{2}\right)^{3 / 2}
$$

as long as $L \geq 10$.

LEMMA 6.2. Let $Y_{1}, \ldots, Y_{k}$ be i.i.d. random variables such that that $\mu=$ $\mathbf{E} Y_{i}<\infty$. Let $Z=\min \left\{Y_{1}, \ldots, Y_{k}\right\}$. Then for any $a<k$, we have $\mathbf{E} Z^{a} \leq(1+$ $\left.\frac{a}{k-a}\right) \mu^{a}$.

PROOF. Simply compute

$$
\begin{aligned}
\mathbf{E} Z^{a} & =\int_{0}^{\infty} \mathbf{P}\left(Y^{a} \geq u\right) d u=\int_{0}^{\infty} \mathbf{P}\left(Y_{1} \geq u^{1 / a}\right)^{k} d u \\
& \leq \int_{0}^{\infty}\left(1 \wedge \frac{\mu}{u^{1 / a}}\right)^{k} d u=\left(1+\frac{a}{k-a}\right) \mu^{a} .
\end{aligned}
$$


COROLlaRY 6.3 (of Proposition 6.1). If $\gamma$ is sufficiently small, then there are constants $C<\infty$ and $b_{\mathrm{pl}}=1+o(1)$ so that for any $K$ and $S$ we have

$$
\mathbf{E} \Psi_{\text {hard }}\left(\left[0,2^{r} S\right) \times\left[0,2^{r+1} S\right)\right) \leq C b_{\mathrm{pl}}^{r} \mathbf{E} \Psi_{\text {hard }}([0, S) \times[0,2 S)) .
$$

Moreover, $b_{\mathrm{pl}}$ can be made arbitrarily close to 1 by making $\gamma$ sufficiently small.

ProOF. By (6.4), in the notation of Proposition 6.1 we have $\mathbf{E} \Psi_{\text {hard }}(\mathfrak{R}) \leq$ $\left(2+o_{K, L}(1)\right) K \mathbf{E} \Psi_{\text {hard }}(\mathfrak{A})$. The statement then follows by induction on the scale after choosing $K, L$ sufficiently large and $\gamma$ sufficiently small.

6.2. Expected geodesic length upper bound. Let $\mathfrak{R}=[0, K S) \times[0, L S)$ with $K=2^{k}$ and $L=2^{l}$. Let $\mathfrak{A}=[0, S) \times[0,2 S)$. We want to show that a left-right crossing of $\mathfrak{R}$ will typically not enter too many dyadic $S \times S$ subboxes of $\mathfrak{R}$. Our strategy will be to show that a path that enters many boxes will likely have a higher weight than the tail-bound value obtained from the "default" paths in Proposition 6.1. Recall the notation $M_{\bullet}$; defined in Section 2.1.2.

Proposition 6.4. For any $u>0$ and $p \in(0,1)$ we have

$$
\begin{gathered}
\mathbf{P}\left[M_{\mathrm{LR} ; S}(\mathfrak{R}) \geq K \max \left\{1,4 u u_{0} c_{\mathrm{PD}} \frac{\mathbf{E} \Psi_{\text {hard }}(\mathfrak{A})}{\Theta_{\text {easy }}(\mathfrak{A})[p]}\right\}\right] \\
\leq u^{-L / 3}+C_{\mathrm{p}} L\left(2 d_{\mathrm{p}}^{2} \sqrt{p}\right)^{K}+o_{K, L}(1) .
\end{gathered}
$$

PROOF. By Proposition 6.1, with probability at least $1-u^{-L / 3}-o_{K, L}(1)$, we have

$$
\Psi_{\mathrm{LR}}(\mathfrak{R}) \leq 2 u K \mathbf{E} \Psi_{\text {hard }}(\mathfrak{A}) .
$$

On the other hand, by Proposition 4.9 and Proposition 4.4, with probability at least $1-C_{\mathrm{p}} L\left(2 d_{\mathrm{p}}^{2} \sqrt{p}\right)^{N}-o_{K, L}(1)$ we have

$$
\min _{\|\pi\|_{S} \geq c_{\mathrm{PD}} N} \psi\left(\pi ; Y_{\mathfrak{R}}\right)>\frac{N}{2 u_{0}} \Theta_{\text {easy }}(\mathfrak{A})[p] .
$$

Thus if

$$
\frac{N}{2 u_{0}} \Theta_{\text {easy }}(\mathfrak{A})[p] \geq 2 u K \mathbf{E} \Psi_{\text {hard }}(\mathfrak{A}),
$$

then with probability at least $1-u^{-L / 3}-C_{\mathrm{p}} L\left(2 d_{\mathrm{p}}^{2} \sqrt{p}\right)^{N}-o_{K, L}(1)$, we have $M_{\mathrm{LR} ; S}(\mathfrak{R}) \leq c_{\mathrm{PD}} N$. Putting

$$
N=K \max \left\{1,4 u_{0} u \frac{\mathbf{E} \Psi_{\text {hard }}(\mathfrak{A})}{\Theta_{\text {easy }}(\mathfrak{A})[p]}\right\}
$$

yields the desired result. 
Proposition 6.5. There is $a \delta_{0}>0$ and a $C_{\mathrm{CL}}>0$ so that the following holds. If $\mathrm{CV}^{2}\left(\Psi_{\text {easy }}(\mathfrak{E})\right)<\delta_{\mathrm{RSW}}$ whenever $\mathfrak{E} \subseteq[0, S) \times[0,2 S)$ has aspect ratio between $1 / 2$ and 2 inclusive, and $\mathrm{CV}^{2}\left(\Psi_{\text {hard }}(\mathfrak{A})\right)<\delta<\delta_{0}$, then we have

$$
\mathbf{E} M_{\mathrm{LR} ; S}(\Re) \leq K\left(C_{\mathrm{CL}}+L\left[2^{-L / 3}+C_{\mathrm{p}} L\left(2 d_{\mathrm{p}}^{2} \sqrt{p_{\mathrm{RSW}}}\right)^{K}\right]\right)+o_{K, L}(1) .
$$

REMARK 6.6. Note that (5.1) implies that the third term decays geometrically as $K \rightarrow \infty$.

PROOF. Putting $p=p_{\text {RSW }}$ in the previous lemma we have, for any $u>0$,

$$
\begin{aligned}
\mathbf{E} M_{\mathrm{LR} ; S}(\mathfrak{R}) \leq & K \max \left\{1,4 u_{0} u c_{\mathrm{PD}} \frac{\mathbf{E} \Psi_{\text {hard }}(\mathfrak{A})}{\Theta_{\text {easy }}(\mathfrak{A})\left[p_{\mathrm{RSW}}\right]}\right\} \\
& +K L\left[u^{-L / 3}+C_{\mathrm{p}} L\left(2 d_{\mathrm{p}}^{2} \sqrt{p_{\mathrm{RSW}}}\right)^{K}\right]+o_{K, L}(1) .
\end{aligned}
$$

Then, since our assumption implies that the hypothesis of Theorem 5.1 holds at scale $S$, putting $u=2$ we obtain

$$
\begin{aligned}
\mathbf{E} M_{\mathrm{LR} ; S}(\mathfrak{R}) \leq & K \max \left\{1,8 u_{0} c_{\mathrm{PD}} \frac{\mathbf{E} \Psi_{\mathrm{hard}}(\mathfrak{A})}{\Theta_{\mathrm{easy}}(\mathfrak{A})\left[p_{\mathrm{RSW}}\right]}\right\} \\
& +K L\left[2^{-L / 3}+C_{\mathrm{p}} L\left(2 d_{\mathrm{p}}^{2} \sqrt{p_{\mathrm{RSW}}}\right)^{K}\right]+o_{K, L}(1) \\
\leq & K \max \left\{1,8 u_{0} c_{\mathrm{PD}} C_{\mathrm{RSW}} \frac{\mathbf{E} \Psi_{\mathrm{hard}}(\mathfrak{A})}{\Theta_{\mathrm{hard}}(\mathfrak{A})\left[p_{\mathrm{RSW}}\right]}\right\} \\
& +K L\left[2^{-L / 3}+C_{\mathrm{p}} L\left(2 d_{\mathrm{p}}^{2}{\sqrt{p_{\mathrm{RSW}}}}^{K}\right]+o_{K, L}(1) .\right.
\end{aligned}
$$

Finally, using the assumption that $\mathrm{CV}^{2}\left(\Psi_{\text {hard }}(\mathfrak{A})\right)<\delta$, if $\delta$ is chosen sufficiently small compared to $p_{\mathrm{RSW}}$, Chebyshev's inequality (or (3.1)) implies the result.

6.3. Diameter upper bound. We now turn our attention to the problem of estimating the point-to-point distance between two points in a box, using a chaining argument to take advantage of our good tail bound established in Proposition 6.1.

Fix a scale $S=2^{s}$. Let $\mathfrak{R}=[0, S) \times[0,2 S)$. For $t \in[0, s]$ and $(i, j) \in\left[0,2^{t}\right)^{2}$, put

$$
\mathfrak{R}_{t ; i, j}= \begin{cases}\left(i \cdot 2^{s-t}, 2 \cdot j \cdot 2^{s-t}\right)+\left[0,2^{s-t}\right) \times\left[0,2 \cdot 2^{s-t}\right), & t \text { even } \\ \left(2 \cdot i \cdot 2^{s-t}, j \cdot 2^{s-t}\right)+\left[0,2 \cdot 2^{s-t}\right) \times\left[0,2^{s-t}\right), & t \text { odd }\end{cases}
$$

For convenience, put $\mathfrak{A}_{t}=\mathfrak{R}_{t ; 0,0}$.

Proposition 6.7. There is a $\delta=\delta_{\text {diam }}>0$ and $C_{\text {diam }}<\infty$, independent of the scale $S$, so that the following holds. If

$$
\mathrm{CV}^{2}\left(\Psi_{\text {hard }}\left(\mathfrak{A}_{t}\right)\right)<\delta
$$


for all $t \geq 0$, and

$$
\mathrm{CV}^{2}\left(\Psi_{\text {easy }}(\mathfrak{A})\right)<\delta_{\text {RSW }}
$$

for all $\mathfrak{A} \subseteq \mathfrak{R}$ of aspect ratio between $1 / 2$ and 2 , inclusive, then, for any $\alpha \in \mathbf{N}$ we have a $C(\alpha) \geq 0$ so that, as long as $\gamma$ is sufficiently small and $u$ is sufficiently large (both compared to $\alpha$ ),

$$
\mathbf{P}\left(\Psi_{\max }(\Re) \geq u \Theta_{\text {easy }}(\Re)\left[q_{\mathrm{pl}}\right]\right) \leq C(\alpha) u^{-\alpha} .
$$

Proof. Let $L \in \mathbf{N}$ be fixed but chosen later. By our crossing distance tail bound (6.2), applied with $L=2^{l}, K=2 L$ and a union bound, for all $u \geq u_{0}$ we have

$$
\begin{gathered}
\mathbf{P}\left[\max _{(i, j) \in\left[0,2^{t}\right)^{2}} \Psi_{\text {hard }}\left(\Re_{t ; i, j}\right) \geq 4 u L \mathbf{E} \Psi_{\text {hard }}\left(\mathfrak{A}_{t+l}\right)\right] \\
\leq\left(1+o_{L}(1)\right) \cdot 4^{t} \cdot u^{-L / 4} .
\end{gathered}
$$

Now we know that, if (6.6) holds and $\delta$ is sufficiently small (compared to $p_{\mathrm{RSW}}$ ), then by (3.1), Theorem 5.1 (noting the hypothesis (6.7)), and Proposition 4.11 (recalling (5.1)) there is a constant $C_{1}$ (depending on $\delta$ ) so that we have

$$
\begin{aligned}
\mathbf{E} \Psi_{\text {hard }}\left(\mathfrak{A}_{t+l}\right) & \leq C_{1} \Theta_{\text {hard }}\left(\mathfrak{A}_{t+l}\right)\left[p_{\mathrm{RSW}}\right] \\
& \leq C_{1} C_{\mathrm{RSW}} \Theta_{\text {easy }}\left(\mathfrak{A}_{t+l}\right)\left[p_{\mathrm{RSW}}\right] \\
& \leq C_{1} C_{\mathrm{pl}} C_{\mathrm{RSW}} a_{\mathrm{pl}}^{t+l} \Theta_{\text {easy }}(\mathfrak{R})\left[q_{\mathrm{pl}}\right] .
\end{aligned}
$$

Combining (6.8) and (6.9) and putting $C=C_{1} C_{\mathrm{pl}} C_{\mathrm{RSW}}$, we get

$$
\begin{gathered}
\left.\mathbf{P} \max _{(i, j) \in\left[0,2^{t}\right)^{2}} \Psi_{\text {hard }}\left(\Re_{t ; i, j}\right) \geq 4 C u L a_{\mathrm{pl}}^{\frac{t+l}{2}} \Theta_{\text {easy }}(\Re)\left[q_{\mathrm{pl}}\right]\right] \\
\leq\left(1+o_{L}(1)\right) \cdot 4^{t} \cdot u^{-L / 4} \cdot a_{\mathrm{pl}}^{L(t+l) / 8} .
\end{gathered}
$$

Using (2.6), we derive

$$
\begin{aligned}
(1+ & \left.o_{L}(1)\right)^{-1} \mathbf{P}\left[\max _{(i, j) \in\left[0,2^{t}\right)^{2}} \Psi_{\text {hard }}\left(\Re_{t ; i, j} ; Y_{\mathfrak{R}}\right) \geq 8 C u L a_{\mathrm{pl}}^{\frac{1}{4}(t+l)} \Theta_{\text {easy }}(\Re)\left[q_{\mathrm{pl}}\right]\right] \\
& \leq 4^{t} u^{-L / 8} a_{\mathrm{pl}}^{L(t+l) / 8}+4^{t} \exp \left(-\omega(1) \frac{\left.\left(\log \left(u a_{\mathrm{pl}}^{-\frac{t+l}{4}}\right)\right)\right)^{2}}{\log 4^{t}}\right) \\
& \leq u^{-L / 8}\left[4^{t} a_{\mathrm{pl}}^{L(t+l) / 8}+\exp \left(t \log 4-\omega_{L}(1) \log u-\omega_{L}(1) \frac{(t+l)^{2}}{t}\right)\right]
\end{aligned}
$$


If we choose $L$ so large and $\gamma$ so small that the term is brackets is summable in $t$, then we can conclude using a union bound that

$$
\begin{aligned}
& \mathbf{P}\left[(\exists t) \max _{(i, j) \in\left[0,2^{t}\right)^{2}} \Psi_{\text {hard }}\left(\Re_{t ; i, j} ; Y_{\mathfrak{R}}\right) \geq 8 C u L a_{\mathrm{pl}}^{\frac{1}{4}(t+l)} \Theta_{\text {easy }}(\mathfrak{R})\left[q_{\mathrm{pl}}\right]\right] \\
& =O_{L}(1) u^{-L / 8} .
\end{aligned}
$$

Now for $x \in \mathfrak{R}$ and $t \in[0, s)$, let $\mathfrak{R}_{t}(x)$ be the $\mathfrak{R}_{t ; i, j}$ containing $x$. Then

$$
\Psi_{x, y}(\Re) \leq \sum_{t \in[0, s]} \Psi_{\text {hard }}\left(\Re_{t}(x) ; Y_{\mathfrak{R}}\right)+\sum_{t \in[1, s]} \Psi_{\text {hard }}\left(\Re_{t}(y) ; Y_{\mathfrak{R}}\right) .
$$

(See Figure 10(a).) This means that

$$
\Psi_{\max }(\Re) \leq 2 \sum_{t \in[0, s]} \max _{(i, j) \in\left[0,2^{t}\right)^{2}} \Psi_{\text {hard }}\left(\Re_{t ; i, j} ; Y_{\mathfrak{R}}\right) ;
$$

this is the chaining argument illustrated in Figure 10(b). Applying (6.10), this implies

$$
\mathbf{P}\left[\Psi_{\max }(\Re) \geq 8 C u L \Theta_{\text {easy }}(\Re)\left[q_{\mathrm{pl}}\right] \sum_{t=0}^{s} a_{\mathrm{pl}}^{\frac{1}{4}(t+l)}\right] \leq O_{L}(1) u^{-L / 8} .
$$

The sum is bounded so we obtain

$$
\mathbf{P}\left[\Psi_{\max }(\Re) \geq u \Theta_{\text {easy }}(\mathfrak{R})\left[q_{\mathrm{pl}}\right]\right] \leq O_{L}(1) u^{-L / 8},
$$

and the result follows since $L$ can be chosen to be arbitrarily large.

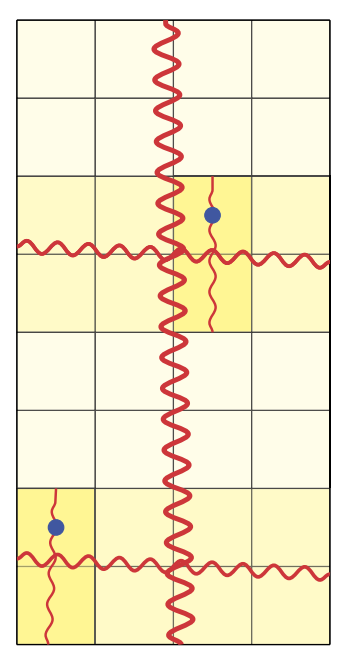

(a) Using two hard crossings at each scale to connect to any two points.

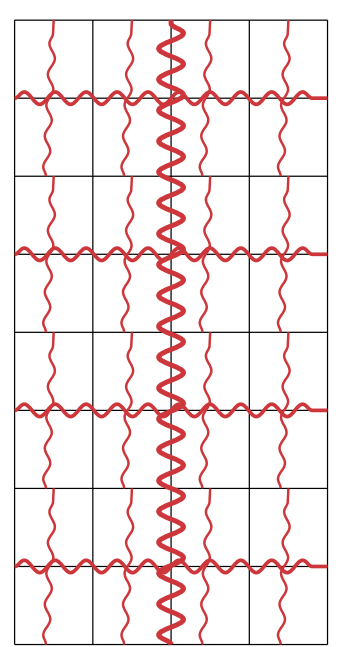

(b) The chaining argument takes the maximum of the hard crossings at each scale.

FIG. 10. Geometric constructions in the proof of Proposition 6.7. 
7. Variation upper bounds. In this section we prove an inductive upper bound on the variance of the crossing distance in a rectangle, which we combine with our lower bounds on the expectation of the crossing distance in order to prove Theorem 3.1.

7.1. Variance of the crossing distance. Our goal in this section is to prove the following bound on the variance of the left-right crossing distance of a rectangle. Let $\Re=[0, K S) \times[0, L S)$.

THEOREM 7.1. For any $\beta>0$, there is $a \delta=\delta_{\mathrm{Var}}>0$ and a constant $C_{\mathrm{Var}}<$ $\infty$ so that if $S, K, L$ are sufficiently large and $\gamma$ is sufficiently small (independent of the scale $S)$, and $\mathrm{CV}^{2}\left(\Psi_{\text {easy }}(\mathfrak{A})\right)<\delta$ whenever $\mathfrak{A} \subseteq[0,3 S)^{2}$ has aspect ratio between $1 / 2$ and 2 inclusive, then

$$
\begin{gathered}
\left(1-o_{K, L}(1)\right) \operatorname{Var}\left(\Psi_{\mathrm{LR}}(\mathfrak{R})\right)-o_{K, L}(1)\left(\mathbf{E} \Psi_{\mathrm{LR}}(\mathfrak{R})\right)^{2} \\
\leq C_{\operatorname{Var}} K L^{2 / \beta}\left(\mathbf{E} \Psi_{\text {easy }}\left([0,3 S)^{2}\right)\right)^{2}
\end{gathered}
$$

The proof of Theorem 7.1 will be be based on the following standard EfronStein inequality [42], which we quote here for reference.

THEOREM 7.2 (Efron-Stein). Let $X_{1}, \ldots, X_{r}, X_{1}^{\prime}, \ldots, X_{r}^{\prime}$ be independent random variables so that $X_{j}$ and $X_{j}^{\prime}$ are identically distributed for each $j$, and $f: \mathbf{R}^{r} \rightarrow \mathbf{R}$. Then

$$
\begin{aligned}
& \operatorname{Var}\left(f\left(X_{1}, \ldots, X_{r}\right)\right) \\
& \quad \leq \frac{1}{2} \sum_{j=1}^{r} \mathbf{E}\left(f\left(X_{1}, \ldots, X_{r}\right)-f\left(X_{1}, \ldots, X_{j-1}, X_{j}^{\prime}, X_{j+1}, \ldots, X_{r}\right)\right)^{2} .
\end{aligned}
$$

To apply Efron-Stein, we need a way to write our field as a function of many independent variables, each of which has only a small effect on the weight of a crossing. We can divide $\bar{\Re}$ into $9 K L$ disjoint dyadic $S \times S$ subboxes, which we will label $\mathfrak{C}_{1}, \ldots, \mathfrak{C}_{9 K L}$ in arbitrary order. Write $Y_{\mathfrak{R}}$ as a function of independent random variables $Z_{1}, \ldots, Z_{9 K L}$ as in Criterion 2.5. For $i=1, \ldots, 9 K L$, write $Y^{\mathfrak{C}_{i}}$ for the field $Y$ with $Z_{i}$ resampled. Theorem 7.2 implies that

$$
\operatorname{Var}\left(\Psi_{\mathrm{LR}}(\Re)\right) \leq \frac{1}{2} \sum_{i=1}^{9 K L} \mathbf{E}\left[\Psi_{\mathrm{LR}}(\Re)-\Psi_{\mathrm{LR}}\left(\Re ; Y^{\mathfrak{C}_{i}}\right)\right]^{2}
$$

We will bound the terms on the right-hand side of (7.2) individually in Lemma 7.4. But first we need the following lemma about the effect of resampling a box on the field distant from that box. Let $\mathfrak{D}_{i}=\overline{\mathfrak{C}_{i}} \cap \mathfrak{R}$ and $\Xi(x, i)=Y^{\mathfrak{C}_{i}}(x)-Y(x)$. 
LEMMA 7.3. Define $\Xi^{*}(i)=\sup _{x \in \mathfrak{B} \backslash \mathfrak{D}_{i}} \Xi(x, i)$. Then for any $a \geq 1$, there is a constant $C_{a}$ (as always, independent of the scale) so that $\mathbf{E}\left|\Xi^{*}(i)\right|^{a} \leq C_{a}$.

Proof. The Borell-TIS inequality (see, e.g., [27], Theorem 7.1, [6], Theorem 6.1 or [1], Theorem 2.1), applied in light of (2.2), tells us that there is a constant $C$ so that

$$
\mathbf{P}\left(\left|\Xi^{*}(i)-\mathbf{E} \Xi^{*}(i)\right| \geq u\right) \leq 2 e^{-\frac{u^{2}}{2 C}} .
$$

Thus we are done as long as we can bound $\Xi^{*}(i)$ by a constant independent of the scale. We do this using Fernique's inequality. By (2.3) we have a constant $C$ so that

$$
\operatorname{Var}(\Xi(x, i)-\Xi(y, i)) \leq \frac{C S^{2}}{[(K \wedge L) S]^{4}}\|x-y\|^{2}=\frac{C\|x-y\|^{2}}{(K \wedge L)^{4} S^{2}} .
$$

Therefore, for a typical point $x$ we have by Fernique's inequality ([19], [1], Theorem 4.1, or [6], Theorem 6.6, as applied in [8], Lemma 3.5) that there exists a constant $C^{\prime}$, independent of $S$, so that $\mathbf{E} \Xi^{*}(i) \leq C^{\prime}$.

LEMMA 7.4. For each $i$, let $E_{i}$ be the event that $\pi_{\mathrm{LR}}(\mathfrak{R}) \cap \mathfrak{D}_{i} \neq \varnothing$. Then we have

$$
\begin{aligned}
\mathbf{E}\left[\Psi_{\mathrm{LR}}\left(\mathfrak{R} ; Y^{\mathfrak{C}_{i}}\right)-\Psi_{\mathrm{LR}}(\mathfrak{R})\right]^{2} \leq & 4 \mathbf{E}\left(\Psi_{\partial}\left(\mathfrak{D}_{i} ; Y^{\mathfrak{C}_{i}}\right) \mathbf{1}_{E_{i}}\right)^{2} \\
& +o_{K, L}(1) \mathbf{E} \Psi_{\text {hard }}([0, S) \times[0,2 S))^{2}
\end{aligned}
$$

PROOF. To begin, note that since $Y$ and $Y^{\mathfrak{C}_{i}}$ are exchangeable, we have

$$
\mathbf{E}\left[\Psi_{\mathrm{LR}}(\mathfrak{R})-\Psi_{\mathrm{LR}}\left(\mathfrak{R} ; Y^{\mathfrak{C}_{i}}\right)\right]^{2}=2 \mathbf{E}\left[0 \vee\left(\Psi_{\mathrm{LR}}(\mathfrak{R})-\Psi_{\mathrm{LR}}\left(\mathfrak{R} ; Y^{\mathfrak{C}_{i}}\right)\right)\right]^{2} .
$$

Let $\pi=\pi_{\mathrm{LR}}(\Re)$. On the occurrence of $E_{i}$, put $\pi=\pi_{0} \cup \pi_{1}$, where $\pi_{0}$ is the part of $\pi$ between the first time $\pi$ enters $\mathfrak{D}_{i}$ and the last time $\pi$ exits $\mathfrak{D}_{i}$, and $\pi_{1}$ is the (generally noncontiguous) set of all other vertices of $\pi$.

Note that

$$
\Psi_{\mathrm{LR}}\left(\Re ; Y^{\mathfrak{C}_{i}}\right)=\inf _{\pi^{\prime}} \sum_{x \in \pi^{\prime}} \exp \left(\gamma Y^{\mathfrak{C}_{i}}(x)\right),
$$

where $\pi^{\prime}$ ranges over all left-right crossings of $\mathfrak{R}$. We claim that

$$
\Psi_{\mathrm{LR}}\left(\mathfrak{R} ; Y^{\mathfrak{C}_{i}}\right)-\Psi_{\mathrm{LR}}(\mathfrak{R}) \leq \sum_{x \in \pi_{1}} e^{\gamma Y(x)}\left[e^{\gamma \Xi(x, i)}-1\right]+\Psi_{\partial}\left(\mathfrak{D}_{i} ; Y^{\mathfrak{C}_{i}}\right) \mathbf{1}_{E_{i}} .
$$

We prove (7.5) by considering separately the situations in which $E_{i}$ does and does not occur.

Case 1. On the event $E_{i}$ we have

$$
\Psi_{\mathrm{LR}}\left(\Re ; Y^{\mathfrak{C}_{i}}\right)=\inf _{\pi^{\prime}} \sum_{x \in \pi^{\prime}} \exp \left(\gamma Y^{\mathfrak{C}_{i}}(x)\right) \leq \psi\left(\pi_{0} ; Y^{\mathfrak{C}_{i}}\right)+\Psi_{x^{*}, y^{*}}\left(\mathfrak{D}_{i} ; Y^{\mathfrak{C}_{i}}\right),
$$


where $\pi^{\prime}$ ranges over all left-right crossings of $\Re$ and $x^{*}$ and $y^{*}$ are the first and last vertices of $\pi_{1}$, respectively. Therefore,

$$
\begin{aligned}
\Psi_{\mathrm{LR}}( & \left.\Re ; Y^{\mathfrak{C}_{i}}\right)-\Psi_{\mathrm{LR}}(\Re ; Y) \\
& \leq \psi\left(\pi_{1} ; Y^{\mathfrak{C}_{i}}\right)+\Psi_{x^{*}, y^{*}}\left(\mathfrak{D}_{i} ; Y^{\mathfrak{C}_{i}}\right)-\psi\left(\pi_{1} ; Y\right)-\psi\left(\pi_{0} ; Y\right) \\
& \leq \psi\left(\pi_{1} ; Y^{\mathfrak{C}_{i}}\right)+\Psi_{\partial}\left(\mathfrak{D}_{i} ; Y^{\mathfrak{C}_{i}}\right)-\psi\left(\pi_{1} ; Y\right) \\
& \leq \Psi_{\partial}\left(\mathfrak{D}_{i} ; Y^{\mathfrak{C}_{i}}\right)+\sum_{x \in \pi_{1}} e^{\gamma Y(x)}\left[1-e^{\gamma \Xi(x, i)}\right] .
\end{aligned}
$$

Case 2. If $E_{i}$ does not occur, then we note that since $\pi$ is a path not passing through $\mathfrak{D}_{i}$,

$$
\begin{aligned}
\psi(\pi ; Y)-\psi\left(\pi ; Y^{\mathfrak{C}_{i}}\right) & =\sum_{x \in \pi}\left[e^{\gamma Y(x)}-e^{\gamma Y^{\mathfrak{C}_{i}(x)}}\right]=\sum_{x \in \pi}\left[e^{\gamma Y(x)}-e^{\gamma Y^{\mathfrak{C}_{i}(x)}}\right] \\
& =\sum_{x \in \pi} e^{\gamma Y(x)}\left[1-e^{\gamma \Xi(x, i)}\right],
\end{aligned}
$$

so we can write

$$
\begin{aligned}
\inf _{\pi^{\prime}} \sum_{x \in \pi^{\prime}} \exp \left(\gamma Y^{\mathfrak{C}_{i}}(x)\right) & \leq \sum_{x \in \pi} \exp \left(\gamma Y^{\mathfrak{C}_{i}}(x)\right) \\
& =\Psi_{\mathrm{LR}}(\Re)+\sum_{x \in \pi} e^{\gamma Y(x)}\left[1-e^{\gamma \Xi(x, i)}\right] .
\end{aligned}
$$

The two cases together imply (7.5). Now, combining (7.4) and (7.5), we have

$$
\begin{aligned}
& \mathbf{E}\left[\left[\Psi_{\mathrm{LR}}(\mathfrak{R})-\Psi_{\mathrm{LR}}\left(\mathfrak{R} ; Y^{\mathfrak{C}_{i}}\right)\right] \vee 0\right]^{2} \\
& \quad \leq \mathbf{E}\left[\sum_{x \in \pi_{1}} e^{\gamma Y(x)}\left[\left[e^{\gamma \Xi(x, i)}-1\right] \vee 0\right]+\Psi_{\partial}\left(\mathfrak{D}_{i} ; Y^{\mathfrak{C}_{i}}\right) \mathbf{1}_{E_{i}}\right]^{2} \\
& \quad \leq 2 \mathbf{E}\left(\sum_{x \in \pi_{1}} e^{\gamma Y(x)}\left[\left[e^{\gamma \Xi(x, i)}-1\right] \vee 0\right]\right)^{2}+2 \mathbf{E}\left(\Psi_{\partial}\left(\mathfrak{D}_{i} ; Y^{\mathfrak{C}_{i}}\right)^{2} \mathbf{1}_{E_{i}}\right) .
\end{aligned}
$$

Considering the first term further we have

$$
\sum_{x \in \pi_{1}} e^{\gamma Y(x)}\left[\left[e^{\gamma \Xi(x, i)}-1\right] \vee 0\right] \leq \Psi_{\mathrm{LR}}(\mathfrak{R}) \cdot \sup _{x \in \mathfrak{R} \backslash \overline{\mathfrak{C}_{i}}}\left[\left[e^{\gamma \Xi_{i}^{*}}-1\right] \vee 0\right],
$$

where $\Xi_{i}^{*}=\sup _{x \in \mathfrak{R} \backslash \overline{\mathfrak{C}_{i}}} \Xi(x, i)$. By Hölder's inequality we have

$$
\begin{aligned}
& \mathbf{E}\left[\Psi_{\mathrm{LR}}(\Re) \cdot \sup _{x \in \mathfrak{R} \backslash \overline{\mathfrak{C}_{i}}}\left[\left[e^{\gamma \Xi_{i}^{*}}-1\right] \vee 0\right]\right]^{2} \\
& \leq\left(\mathbf{E} \sup _{x \in \mathfrak{R} \backslash \overline{\mathfrak{C}_{i}}}\left[\left[e^{\gamma \Xi_{i}^{*}}-1\right]^{3 / 2} \vee 0\right]\right)^{4 / 3}\left(\mathbf{E} \Psi_{\mathrm{LR}}(\mathfrak{R})^{3}\right)^{2 / 3} \\
& \leq o_{K, L}(1) \mathbf{E} \Psi_{\text {hard }}([0, S) \times[0,2 S))^{2}
\end{aligned}
$$

with the second inequality by (6.5) and Lemma 7.3. Then (7.3) follows. 
Now we can prove our variance bound.

Proof of Theorem 7.1. Let $q^{\prime} \in\left(q_{\mathrm{pl}}, 1\right)$. Note that we can split the event $\Psi_{\partial}\left(\mathfrak{D}_{i} ; Y^{\mathfrak{C}_{i}}\right)^{2} \mathbf{1}_{E_{i}}$ into cases as follows:

$$
\begin{aligned}
\Psi_{\partial}\left(\mathfrak{D}_{i} ; Y^{\mathfrak{C}_{i}}\right)^{2} \mathbf{1}_{E_{i}} & \\
= & \Psi_{\partial}\left(\mathfrak{D}_{i} ; Y^{\mathfrak{C}_{i}}\right)^{2} \mathbf{1}_{E_{i}} \mathbf{1}\left\{\Psi_{\partial}\left(\mathfrak{D}_{i} ; Y^{\mathfrak{C}_{i}}\right) \geq u \Theta_{\text {easy }}\left(\mathfrak{D}_{i} ; Y^{\mathfrak{C}_{i}}\right)\left[q^{\prime}\right]\right\} \\
& +\Psi_{\partial}\left(\mathfrak{D}_{i} ; Y^{\mathfrak{C}_{i}}\right)^{2} \mathbf{1}_{E_{i}} \mathbf{1}\left\{\Psi_{\partial}\left(\mathfrak{D}_{i} ; Y^{\mathfrak{C}_{i}}\right)<u \Theta_{\text {easy }}\left(\mathfrak{D}_{i} ; Y^{\mathfrak{C}_{i}}\right)\left[q^{\prime}\right]\right\} \\
\leq & \Psi_{\partial}\left(\mathfrak{D}_{i} ; Y^{\mathfrak{C}_{i}}\right)^{2} \mathbf{1}\left\{\Psi_{\partial}\left(\mathfrak{D}_{i} ; Y^{\mathfrak{C}_{i}}\right) \geq u \Theta_{\text {easy }}\left(\mathfrak{D}_{i} ; Y^{\mathfrak{C}_{i}}\right)\left[q^{\prime}\right]\right\} \\
& +u^{2} \Theta_{\text {easy }}\left(\mathfrak{D}_{i} ; Y^{\mathfrak{C}_{i}}\right)\left[q^{\prime}\right]^{2} \mathbf{1}_{E_{i}} .
\end{aligned}
$$

Moreover, we have by (2.5) and Proposition 6.7, as long as $u$ is sufficiently large,

$$
\begin{aligned}
\mathbf{E}\left[\Psi_{\partial}\right. & \left.\left(\mathfrak{D}_{i} ; Y^{\mathfrak{C}_{i}}\right)^{2} \mathbf{1}\left\{\Psi_{\partial}\left(\mathfrak{D}_{i} ; Y^{\mathfrak{C}_{i}}\right) \geq u \Theta_{\text {easy }}\left(\mathfrak{D}_{i} ; Y^{\mathfrak{C}_{i}}\right)\left[q^{\prime}\right]\right\}\right] \\
\leq & (1+o(1)) \mathbf{E}\left[\Psi_{\partial}\left(\mathfrak{D}_{i}\right)^{2} \mathbf{1}\left\{\Psi_{\partial}\left(\mathfrak{D}_{i}\right) \geq \frac{1}{2} u \Theta_{\text {easy }}\left(\mathfrak{D}_{i}\right)\left[q_{\mathrm{pl}}\right]\right\}\right] \\
& \leq O_{\alpha}(1) \cdot \Theta_{\text {easy }}\left(\mathfrak{D}_{i}\right)\left[q_{\mathrm{pl}}\right]^{2} \cdot \int_{u / 2}^{\infty} v^{2-\alpha} d v \\
& =O_{\alpha}(1) \cdot \Theta_{\text {easy }}\left(\mathfrak{D}_{i}\right)\left[q_{\mathrm{pl}}\right]^{2} \cdot u^{3-\alpha} .
\end{aligned}
$$

Also, by (3.2), as long as $\delta$ is sufficiently small we have

$$
\Theta_{\text {easy }}\left(\mathfrak{D}_{i} ; Y^{\mathfrak{C}_{i}}\right)\left[q^{\prime}\right]^{2} \leq O(1) \cdot \Theta_{\text {easy }}\left(\mathfrak{D}_{i} ; Y^{\mathfrak{C}_{i}}\right)\left[q_{\mathrm{pl}}\right]^{2} .
$$

Combining Lemma 7.4, (7.6), (7.7), (7.8) and Proposition 6.5, and assuming that $K$ and $L$ are sufficiently large and $\delta, \gamma$ sufficiently small, we have

$$
\begin{aligned}
& \frac{1}{2} \sum_{i=1}^{9 K L} \mathbf{E}\left[\Psi_{\mathrm{LR}}(\Re ; Y)-\Psi_{\mathrm{LR}}\left(\Re ; Y^{\mathfrak{C}_{i}}\right)\right]^{2} \\
& \leq \frac{1}{2} \sum_{i=1}^{9 K L} 4\left(\mathbf{E}\left(\Psi_{\partial}\left(\mathfrak{D}_{i} ; Y^{\mathfrak{C}_{i}}\right)^{2} \mathbf{1}_{E_{i}}\right)+o_{K, L}(1) \mathbf{E} \Psi_{\mathrm{LR}}(\Re)^{2}\right) \\
& \leq \sum_{i=1}^{9 K L} O_{\alpha}(1) \Theta_{\text {easy }}\left(\mathfrak{D}_{i}\right)\left[q_{\mathrm{pl}}\right]^{2} u^{3-\alpha}+\frac{1}{2} u^{2} \Theta_{\text {easy }}\left([0,3 S)^{2}\right)\left[q_{\mathrm{pl}}\right]^{2} \mathbf{E} M_{\mathrm{LR} ; S}(\Re) \\
&+o_{K, L}(1) \mathbf{E} \Psi_{\text {hard }}([0, S) \times[0,2 S))^{2} \\
& \leq O_{\beta}(1) K L \Theta_{\text {easy }}\left([0,3 S)^{2}\right)\left[q_{\mathrm{pl}}\right]^{2} u^{-\beta}+C_{\mathrm{CL}} K u^{2} \Theta_{\text {easy }}\left([0,3 S)^{2}\right)\left[q_{\mathrm{pl}}\right]^{2} \\
&+o_{K, L}(1) \mathbf{E} \Psi_{\text {hard }}([0, S) \times[0,2 S))^{2}
\end{aligned}
$$


where $\beta=\alpha-3$. Then if we put $u=L^{1 / \beta}$, then we obtain

$$
\begin{aligned}
\operatorname{Var} \Psi_{\mathrm{LR}}(\Re) \leq & \frac{1}{2} \sum_{i=1}^{9 K L} \mathbf{E}\left[\Psi_{\mathrm{LR}}(\Re)-\Psi_{\mathrm{LR}}\left(\mathfrak{R} ; Y^{\mathfrak{C}_{i}}\right)\right]^{2} \\
\leq & K \Theta_{\text {easy }}\left([0,3 S)^{2}\right)\left[q_{\mathrm{pl}}\right]^{2}\left[C_{\mathrm{CL}} L^{2 / \beta}+O_{\beta}(1)\right] \\
& +o_{K, L}(1) \mathbf{E} \Psi_{\text {hard }}([0, S) \times[0,2 S))^{2}
\end{aligned}
$$

Then (7.1) follows from another application of (3.1), along with the hypothesis on the coefficient of variation and Theorem 5.1 and Lemma 3.2 to bound the last term in the last equation.

7.2. Coefficient of variation. Armed with our inductive upper bound on crossing distance variance from the previous subsection, and inductive lower bound on expected crossing distance from Section 4, we are now ready to work toward a proof of Theorem 3.1 by induction.

LEMMA 7.5. There is a $\delta_{0}>0$ so that if $0<\delta<\delta_{0}$ then the following holds. Fix a scale $S=2^{s}$. Suppose that

$$
\mathrm{CV}^{2}\left(\Psi_{\mathrm{LR}}(\mathfrak{A})\right)<\delta
$$

for all $\mathfrak{A} \subseteq[0, S) \times[0,2 S)$ of aspect ratio between $1 / 2$ and 2 , inclusive. If $K$ is sufficiently large compared to $\delta$ and $K / 2 \leq L \leq 2 K$ and $\gamma$ is sufficiently small compared to $\delta, K$ and $L$, then if $\Re=[0, K S) \times[0, L S)$, we have

$$
\mathrm{CV}^{2}\left(\Psi_{\mathrm{LR}}(\mathfrak{R})\right)<\delta .
$$

PROOF. By Theorem 7.1, if $K$ and $L$ are sufficiently large, we have

$$
\begin{gathered}
\left(1-o_{K, L}(1)\right) \cdot \operatorname{Var}\left(\Psi_{\mathrm{LR}}(\mathfrak{R})\right)-o_{K, L}(1)\left(\mathbf{E} \Psi_{\mathrm{LR}}(\mathfrak{R})\right)^{2} \\
\leq C_{\mathrm{Var}} \cdot K \cdot L^{2 / \beta} \cdot\left(\mathbf{E} \Psi_{\text {easy }}\left([0,3 S)^{2} ; Y^{\mathfrak{C}_{i}}\right)\right)^{2} .
\end{gathered}
$$

Moreover, by Corollary 4.10, we have

$$
\mathbf{E} \Psi_{\mathrm{LR}}(\mathfrak{R}) \geq \frac{K}{2 u_{0}} \Theta_{\text {easy }}(\mathfrak{A})\left[p_{\mathrm{RSW}}\right] \cdot\left(1-C_{\mathrm{p}} L\left(2 d_{\mathrm{p}}^{2} \sqrt{p_{\mathrm{RSW}}}\right)^{K}-o_{K, L}(1)\right),
$$

so (again recalling (5.1)) if $K$ and $L$ are sufficiently large and $\gamma$ is sufficiently small then we have

$$
\mathbf{E} \Psi_{\mathrm{LR}}(\mathfrak{R}) \geq \frac{K}{4 u_{0}} \Theta_{\text {easy }}(\mathfrak{A})\left[p_{\mathrm{RSW}}\right] .
$$


Therefore, we have a constant $C$ so that

$$
\begin{aligned}
\mathrm{CV}^{2}\left(\Psi_{\mathrm{LR}}(\mathfrak{R})\right) & =\frac{\operatorname{Var}\left(\Psi_{\mathrm{LR}}(\mathfrak{R})\right)}{\left(\mathbf{E} \Psi_{\mathrm{LR}}(\mathfrak{R})\right)^{2}} \\
& \leq \frac{C_{\mathrm{Var}} K L^{2 / \beta}\left(\mathbf{E} \Psi_{\text {easy }}(\mathfrak{A})\right)^{2}}{\left(1-o_{K, L}(1)\right) \cdot K^{2}\left(\Theta_{\text {easy }}(\mathfrak{A})\left[p_{\mathrm{RSW}}\right]\right)}+o_{K, L}(1) \\
& \leq \frac{C L^{2 / \beta}}{K}+o_{K, L}(1)
\end{aligned}
$$

If we choose $K$ sufficiently large compared to $\delta$, and $\beta$ sufficiently large, then this yields $\mathrm{CV}^{2}\left(\Psi_{\mathrm{LR}}(\mathfrak{R})\right)<\delta$ for all $K / 2 \leq L \leq 2 K$.

LEMMA 7.6. For a fixed scale $S_{0}$ we have $\mathrm{CV}^{2}\left(\Psi_{\mathrm{LR}}(\mathfrak{A})\right)=o_{S_{0}}(1)$ for all $\mathfrak{A} \subset\left[0, S_{0}\right) \times\left[0,2 S_{0}\right)$.

ProOF. Without loss of generality, let $\mathfrak{A}=[0, S] \times[0, T]$. We note that $\Psi_{\mathrm{LR}}(\mathfrak{A}) \leq \psi\left(\pi_{0} ; Y_{\mathfrak{A}}\right)$, where $\pi_{0}$ is a straight-line path across $\mathfrak{A}$. Therefore,

$$
\mathbf{E} \Psi_{\mathrm{LR}}(\mathfrak{A})^{2} \leq \mathbf{E} \psi\left(\pi_{0} ; Y_{\mathfrak{A}}\right)^{2}=S^{2}+o_{S_{0}}(1) .
$$

On the other hand,

$$
\Psi_{\mathrm{LR}}(\mathfrak{A}) \geq S \min _{x \in \mathfrak{A}} \exp (\gamma Y(x))
$$

so

$$
\mathbf{E} \Psi_{\mathrm{LR}}(\mathfrak{A}) \geq S \mathbf{E}\left[\min _{x \in \mathfrak{A}} \exp (\gamma Y(x))\right]=S+o_{S_{0}}(1)
$$

Therefore,

$$
\mathrm{CV}^{2}\left(\Psi_{\mathrm{LR}}(\mathfrak{A})\right) \leq \frac{\mathbf{E} \Psi_{\mathrm{LR}}(\mathfrak{A})^{2}-\left(\mathbf{E} \Psi_{\mathrm{LR}}(\mathfrak{A})\right)^{2}}{\left(\mathbf{E} \Psi_{\mathrm{LR}}(\mathfrak{A})\right)^{2}}=o_{S_{0}}(1)
$$

We have now assembled all of the pieces necessary for the proof of Theorem 3.1.

Proof of Theorem 3.1. Apply Lemma 7.6 for some $S_{0}>K$, with $K$ chosen large enough compared to $\delta$ to satisfy the assumptions of Lemma 7.5. Then inductively applying Lemma 7.5 allows us to bound the coefficient of variation of every box of the given aspect ratios.

8. Subsequential limits of FPP metrics. All of the necessary estimates in hand, we now proceed to establish existence and continuity properties of the scaling limit metrics of Liouville FPP. 
8.1. Tightness and subsequential convergence. As a corollary of Theorem 3.1, we will derive a tightness result for the first-passage percolation metric, properly scaled.

For each $S=2^{s}$, let $\Re_{s}=[0, S)^{2}$. For $x, y \in[0,1]_{\mathbf{R}}^{2} \cap \frac{1}{2^{s}} \mathbf{Z}^{2}$, let

$$
d_{s}(x, y)=\frac{\Psi_{S x, S y}\left(\Re_{s}\right)}{\Theta_{\text {easy }}\left(\mathfrak{R}_{s}\right)\left[q_{\mathrm{pl}}\right]} .
$$

For arbitrary $x, y \in[0,1]_{\mathbf{R}}^{2}$, define $d_{s}(x, y)$ by linear interpolation, namely (as in (4.2) of [30])

$$
\begin{aligned}
d_{S}(x, y)= & (\lceil S x\rceil-S x)(\lceil S y\rceil-S y) d_{s}\left(\frac{1}{S}\lfloor S x\rfloor, \frac{1}{S}\lfloor S y\rfloor\right) \\
& +(\lceil S x\rceil-S x)(S y-\lfloor S y\rfloor) d_{s}\left(\frac{1}{S}\lfloor S x\rfloor, \frac{1}{S}\lceil S y\rceil\right) \\
& +(S y-\lfloor S x\rfloor)(\lceil S y\rceil-S y) d_{S}\left(\frac{1}{S}\lceil S x\rceil, \frac{1}{S}\lfloor S y\rfloor\right) \\
& +(S y-\lfloor S x\rfloor)(S y-\lfloor S y\rfloor) d_{S}\left(\frac{1}{S}\lceil S x\rceil, \frac{1}{S}\lceil S y\rceil\right) .
\end{aligned}
$$

THEOREM 8.1. If $\gamma$ is sufficiently small, then the sequence $\left\{d_{s}\right\}_{s \in \mathbf{N}}$ is tight in the Gromov-Hausdorff topology.

Note that the first part of Theorem 1.1 follows from Theorem 8.1 by Prokhorov's theorem.

PROPOSITION 8.2. There exists $\xi>0$ so that, if $\gamma$ is sufficiently small then for any $\varepsilon>0$, there exists $C(\varepsilon)>0$ such that, for each $S=2^{s}$, the probability is at most $\varepsilon$ that there exists a dyadic square $\mathfrak{C} \subset[0,1]_{\mathbf{R}}^{2}$ such that $\operatorname{diam}_{d_{s}}\left(\mathfrak{C} \cap \frac{1}{S} \mathbf{Z}^{2}\right) \geq$ $C(\varepsilon)\left(\operatorname{diam}_{\|\cdot\|_{\infty}} \mathfrak{C}\right)^{\xi}$, where $\|\cdot\|_{\infty}$ denotes the max norm.

Proof. Let $\mathfrak{B}=[0, S)^{2}$ and let $\mathfrak{C}$ be a dyadic $T \times T$ square contained in $\mathfrak{B}$ where $T=2^{t}$. By Proposition 6.7, as long as $\delta$ is sufficiently small (and $\gamma$ is chosen small enough, in particular so that Theorem 3.1 holds for $\delta$ ) we have a $C$ (independent of the scale) so that

$$
\mathbf{P}\left(\Psi_{\max }(\mathfrak{C}) \geq u \Theta_{\text {easy }}(\mathfrak{C})\left[q_{\mathrm{pl}}\right]\right) \leq C u^{-\alpha}
$$

for any dyadic square $\mathfrak{C} \subset \mathfrak{B}$. This means that, using Proposition 4.11 and (3.2), we have

$$
\begin{aligned}
& \mathbf{P}\left(\Psi_{\max }(\mathfrak{C}) \geq u \Theta_{\text {easy }}(\mathfrak{B})\left[q_{\mathrm{pl}}\right]\right) \\
& \quad=\mathbf{P}\left(\Psi_{\max }(\mathfrak{C}) \geq u \frac{\Theta_{\text {easy }}(\mathfrak{B})\left[q_{\mathrm{pl}}\right]}{\Theta_{\text {easy }}(\mathfrak{C})\left[q_{\mathrm{pl}}\right]} \Theta_{\text {easy }}(\mathfrak{C})\left[q_{\mathrm{pl}}\right]\right) \leq C_{\alpha}^{\prime} u^{-\alpha} a_{\mathrm{pl}}^{\alpha(s-t)} .
\end{aligned}
$$


(Recall from Proposition 4.11 that $a_{\mathrm{pl}} \in(0,1)$, so the right-hand side is a decreasing function of $s-t$.) Putting

$$
u=v a_{\mathrm{pl}}^{\beta(s-t)}
$$

for some $\beta \in(0,1)$ to be chosen, this yields

$$
\mathbf{P}\left(\Psi_{\max }(\mathfrak{C}) \geq v a_{\mathrm{pl}}^{\beta(s-t)} \Theta_{\text {easy }}(\mathfrak{B})\left[q_{\mathrm{pl}}\right]\right) \leq C_{\alpha}^{\prime} v^{-\alpha} a_{\mathrm{pl}}^{\alpha(1-\beta)(s-t)} .
$$

Moreover, we have, for $0<\beta^{\prime}<\beta$ (using (2.6)),

$$
\begin{aligned}
\mathbf{P}\left(\Psi_{\max }\right. & \left.\left(\mathfrak{C} ; Y_{\mathfrak{B}}\right) \geq v a_{\mathrm{pl}}^{\beta^{\prime}(s-t)} \Theta_{\text {easy }}(\mathfrak{B})\left[q_{\mathrm{pl}}\right]\right) \\
\leq & \mathbf{P}\left(\Psi_{\max }(\mathfrak{C}) \geq \sqrt{v} a_{\mathrm{pl}}^{\beta(s-t)} \Theta_{\text {easy }}(\mathfrak{B})\left[q_{\mathrm{pl}}\right]\right) \\
& +\exp \left(-\omega(1) \cdot \frac{\left(\log \left(\sqrt{v} \cdot a_{\mathrm{pl}}^{\left(\beta-\beta^{\prime}\right)(s-t)}\right)\right)^{2}}{s-t}\right) \\
\leq & C_{\alpha}^{\prime} v^{-\alpha / 2} a_{\mathrm{pl}}^{\alpha(1-\beta)(s-t)}+\exp \left(-\omega(1) \cdot\left[\left(\beta-\beta^{\prime}\right)^{2}(s-t)+\log v\right]\right) .
\end{aligned}
$$

Therefore, using a union bound, the probability that there exists a dyadic square $\mathfrak{C} \subset \mathfrak{B}$ such that $\Psi_{\max }(\mathfrak{C} ; X) \geq v a_{\mathrm{pl}}^{\beta^{\prime}(s-t)} \Theta_{\text {easy }}(\mathfrak{B})\left[q_{\mathrm{pl}}\right]$ is bounded by

$$
C_{\alpha}^{\prime} v^{-\alpha / 2} \sum_{t=0}^{s} 4^{s-t}\left(a_{\mathrm{pl}}^{\alpha(1-\beta)(s-t)}+\exp \left(-\omega(1)\left[\left(\beta-\beta^{\prime}\right)^{2}(s-t)+\log v\right]\right)\right) .
$$

If we choose $\alpha$ large enough and $\gamma$ small enough (but both fixed), then the sum on the right is bounded in $s$, and so the right-hand side can be made arbitrarily small, uniformly in $s$, by increasing $v$. Now note that

$$
a_{\mathrm{pl}}^{\beta^{\prime}(s-t)}=e^{-\beta^{\prime} \log _{2}(T / S) \log a_{\mathrm{pl}}}=e^{-\beta^{\prime} \log (T / S) \log _{2} a_{\mathrm{pl}}}=(T / S)^{\beta^{\prime} \log _{2}\left(1 / a_{\mathrm{pl}}\right)} .
$$

Therefore, the probability is at most $C_{\alpha}^{\prime \prime} v^{-\alpha / 2}$ that there exists a dyadic square $\mathfrak{C} \subset[0,1]_{\mathbf{R}}^{2}$, of side length at least $1 / S$, such that $\operatorname{diam}_{d_{s}}\left(\mathfrak{C} \cap \frac{1}{S} \mathbf{Z}^{2}\right) \geq v \times$ $\left(\operatorname{diam}_{\|\cdot\|_{\infty}} \mathfrak{C}\right)^{\beta^{\prime} \log _{2}\left(1 / a_{\mathrm{pl}}\right)}$. Since this independent of $S$, the proof is complete [with $\xi=\beta^{\prime} \log _{2}\left(1 / a_{\mathrm{pl}}\right)$ and $C(\varepsilon)=v$ chosen large enough so that $\left.C_{\alpha}^{\prime \prime} v^{-\alpha / 2}<\varepsilon\right]$.

COROLLARY 8.3. There exists a $\xi>0$ so that if $\gamma$ is sufficiently small then the following holds. For any $\varepsilon>0$, there exists $C(\varepsilon)>0$ such that, for each $S=2^{s}$, the probability is at most $\varepsilon$ that there exists a dyadic square $\mathfrak{C} \subset[0,1]_{\mathbf{R}}^{2}$ such that $\operatorname{diam}_{d_{s}}(\mathfrak{C}) \geq C(\varepsilon)\left(\operatorname{diam}_{\|\cdot\|_{\infty}} \mathfrak{C}\right)^{\xi}$.

PROOF. Hölder conditions are preserved under the linear interpolation scheme (8.1). 
COROLlARY 8.4. If $\gamma$ is sufficiently small then the following holds. For any $\varepsilon>0$, there exists $C^{\prime}(\varepsilon)>0$ such that, for each $S=2^{s}$, we have

$$
\mathbf{P}\left(\text { there exist } x, y \in[0,1]_{\mathbf{R}}^{2} \text { s.t. } d_{S}(x, y) \geq C^{\prime}(\varepsilon) \cdot\|x-y\|_{\infty}^{\xi}\right) \leq \varepsilon
$$

with $\xi$ as above.

Proof. Any two $x, y \in[0,1]_{\mathbf{R}}^{2}$ are contained within one or two adjacent dyadic boxes of side length at most twice $\|x-y\|_{\infty}$. Then the result follows from Corollary 8.3 .

We are now ready to prove our theorem.

PROOF OF THEOREM 8.1. By Corollary 8.4 and the compact embedding of Hölder spaces, for each $\varepsilon>0$ and $\xi^{\prime}<\xi$ there is a compact set $A_{\varepsilon}$ in the Holder$\xi$ ' topology of Hölder- $\xi$ functions on $[0,1]^{4}$ so that $\mathbf{P}\left(d_{s} \notin A_{\varepsilon}\right)<\varepsilon$. Since the Gromov-Hausdorff topology is weaker than the uniform topology, which is in turn weaker than the Hölder- $\xi$ topology (see, e.g., [30], Proposition 3.3.2), $A_{\varepsilon}$ is also compact in the Gromov-Hausdorff topology. This implies that $\left\{d_{s}\right\}$ is tight with respect to the Gromov-Hausdorff topology.

8.2. Hölder-continuity of limiting metrics. In this section we prove that $[0,1]_{\mathbf{R}}^{2}$, equipped with the topology induced by any limit point metric, is homeomorphic to $[0,1]_{\mathbf{R}}^{2}$ with the standard topology by a Hölder-continuous homeomorphism with Hölder-continuous inverse. In fact, one of the necessary maps was obtained in the coarse of the proof in the previous section. The other direction follows from a similar chaining argument, but using lower bounds instead of upper bounds.

Proposition 8.5. Any limit point of $\left\{d_{s}\right\}$ is almost surely Hölder- $\xi^{\prime}$ continuous with respect to the Euclidean metric for any $\xi^{\prime}<\xi$ as in Proposition 8.2.

PROOF. Follows from the proof of Theorem 8.1.

PROPOSITION 8.6. If $\gamma$ is sufficiently small, then there exists a $\xi^{\prime}>1$ so that for all $\varepsilon>0$ there exist $C(\varepsilon)>0$ such that for any scale $s$ we have

$$
\mathbf{P}\left(\text { there exist }(x, y) \in[0,1]_{\mathbf{R}}^{2} \text { s.t. } d_{S}(x, y) \leq \frac{1}{C(\varepsilon)}\|x-y\|_{\infty}^{\xi^{\prime}}\right) \leq \varepsilon .
$$

Moreover, we can take $\xi^{\prime} \rightarrow 1$ as $\gamma \rightarrow 0$. 
Proof. We will use the notation $S=2^{s}$ and $T=2^{t}$ throughout. Let $\mathfrak{R}=$ $[0, S)^{2}$. Fix a scale $t<s$. Let $\mathfrak{A}_{t}=[0, T) \times[0,2 T)$. By Proposition 4.9, for any $p \in(0,1 / 2)$ we have

$$
\begin{aligned}
& \mathbf{P}\left[\min _{|\mathcal{P}(\pi)| \geq N} \psi\left(\pi ; Y_{\mathfrak{R}}\right) \leq \frac{N}{2 u} \Theta_{\text {easy }}\left(\mathfrak{A}_{t}\right)[p]\right] \\
& \leq(S / T)^{2}\left[O(1)\left(2 d_{\mathrm{p}}^{2} \sqrt{p}\right)^{N}+\exp \left(-\omega(1) \cdot \frac{(\log u)^{2}}{s-t}\right)\right],
\end{aligned}
$$

where in the notation $\mathcal{P}(\pi)$ we consider passes of size $2 T \times T$ and $T \times 2 T$. Fixing $0<\beta^{\prime}<\beta<1$ and summing over all scales and putting $N=(S / T)^{\beta} v$, $u=(S / T)^{\beta^{\prime}} v^{2}$, this gives, whenever $u \geq u_{0}$,

$$
\begin{aligned}
& \mathbf{P}\left[(\exists t \in[0, s)) \min _{\left|\mathcal{P}_{T}(\pi)\right| \geq\left(\frac{S}{T}\right)^{\beta} v} \psi\left(\pi ; Y_{\mathfrak{R}}\right) \leq \frac{1}{2 v}\left(\frac{S}{T}\right)^{\beta-\beta^{\prime}} \Theta_{\text {easy }}\left(\mathfrak{A}_{t}\right)[p]\right] \\
& \leq \sum_{t=0}^{s-1}\left(\frac{S}{T}\right)^{2}\left[O(1)\left(2 d_{\mathrm{p}}^{2} \sqrt{p}\right)^{(S / T)^{\beta} v}+e^{-\omega(1) \cdot(s-t+\log v)}\right] .
\end{aligned}
$$

As long as $v$ is large enough and $p$ and $\gamma$ are small enough, the last sum is finite as $s \rightarrow \infty$ and goes to 0 , uniformly in $s$, as $v \rightarrow \infty$.

By Corollary 6.3, Theorem 3.1 and Theorem 5.1, as long as $\gamma$ and $\delta$ are sufficiently small relative to $p$ we have

$$
\frac{\Theta_{\text {easy }}(\mathfrak{R})[p]}{\Theta_{\text {easy }}\left(\mathfrak{A}_{t}\right)[p]} \leq C(S / T)^{1+o(1)} .
$$

Therefore, we obtain $\lim _{v \rightarrow \infty} \mathbf{P}\left[E_{v}\right]=0$ uniformly in $s$, where $E_{v}$ is the event that there exists a $t \in[0, s)$ such that

$$
\min _{|\mathcal{P}(\pi)| \geq(S / T)^{\beta} v} \psi\left(\pi ; Y_{\mathfrak{R}}\right) \leq \frac{1}{2 v}\left(\frac{S}{T}\right)^{\beta-\beta^{\prime}-1-o(1)} \Theta_{\text {easy }}(\mathfrak{R})[p],
$$

where again $\mathcal{P}(\pi)$ considers passes of size $2 T \times T$ and $T \times 2 T$. Using the normalized metric $d_{s}$, we see that $E_{v}$ contains the event that there exist a $t \in[0, s)$ and $x_{1}, x_{2} \in[0,1]_{\mathbf{R}}^{2} \cap \frac{1}{S} \mathbf{Z}^{2}$ such that both

$$
\left\|x_{1}-x_{2}\right\|_{\infty} \geq c_{\mathrm{PD}}^{-1} v\left(\frac{T}{S}\right)^{1-\beta}
$$

and

$$
d_{S}\left(x_{1}, x_{2}\right) \leq \frac{1}{2 v}\left(\frac{T}{S}\right)^{1-\left(\beta-\beta^{\prime}\right)+o(1)} .
$$

This means that there are constants $C^{\prime}, C^{\prime \prime}$ so that, with probability going to 1 as $v \rightarrow \infty$, for all $x_{1}, x_{2} \in[0,1]_{\mathbf{R}}^{2} \cap \frac{1}{C^{\prime} S} \mathbf{Z}^{2}$ we have

$$
d_{s}\left(x_{1}, x_{2}\right) \geq \frac{C}{v^{2+\alpha+o(1)}}\left\|x_{1}-x_{2}\right\|_{\infty}^{1+\alpha+o(1)},
$$


where $\alpha=\beta^{\prime} /(1-\beta)$. Since this property is preserved (up to constants) by the linear interpolation, we in fact have (8.3) for all $x_{1}, x_{2} \in[0,1]_{\mathbf{R}}^{2}$ and all scales $s$. By choosing $\beta, \beta^{\prime}$ appropriately, we can make $\alpha$ arbitrarily small as long as $\gamma$ is small enough. This completes the proof of the proposition.

PROPOSITION 8.7. Any limit point $d$ of $\left\{d_{s}\right\}$ almost surely has the property that

$$
d(x, y) \geq \frac{1}{C}\|x-y\|_{\infty}^{\xi^{\prime}}
$$

for some constant $\xi^{\prime} \in(0,1)$ and some (random) $C$.

PROOF. Let

$$
C_{s}=\sup _{x, y \in[0,1]_{\mathbf{R}}^{2}} \frac{\|x-y\|_{\infty}^{\xi^{\prime}}}{d_{s}(x, y)} .
$$

By Proposition 8.6, $C_{s}<\infty$ almost surely, and moreover the sequence $\left\{C_{s}\right\}_{s}$ is tight. This means that the sequence $\left\{\left(d_{S}, C_{s}\right)\right\}_{S}$, where the space of metrics is given the uniform topology, is tight as well, so $\left\{\left(d_{s}, C_{s}\right)\right\}_{s}$ converges along subsequences. By the Skorohod representation theorem (noting that $C^{\infty}\left([0,1]^{4}\right) \times \mathbf{R}$ is a separable Fréchet space), we can put all of the $\left(d_{s}, C_{s}\right)$ s on a common probability space and get almost-sure convergence along subsequences. But convergence along an almost-surely convergent subsequence preserves bounds of the form (8.4), and such a bound holds for $d_{s}$ along any almost-surely convergent subsequence of $\left\{\left(d_{s}, C_{s}\right)\right\}_{S}$ since in such a case the $C_{s}$ s will be bounded. Thus the proposition is proved.

The second statement of Theorem 1.1 is the combination of the results of Proposition 8.5 and Proposition 8.7.

Acknowledgments. We thank Steve Lalley for encouragement and useful discussions, and an anonymous referee for a great number of helpful comments.

\section{REFERENCES}

[1] Adler, R. J. (1990). An Introduction to Continuity, Extrema, and Related Topics for General Gaussian Processes. Institute of Mathematical Statistics Lecture Notes-Monograph Series 12. IMS, Hayward, CA. MR 1088478

[2] Ahlberg, D., TAssion, V. and Teixeira, A. (2017). Sharpness of the phase transition for continuum percolation in $\mathbb{R}^{2}$. Probab. Theory Related Fields 172 525-581. MR3851838

[3] Auffinger, A., Damron, M. and Hanson, J. (2017). 50 Years of First-Passage Percolation. University Lecture Series 68. Amer. Math. Soc., Providence, RI. MR3729447

[4] Beffara, V. and Duminil-Copin, H. (2012). The self-dual point of the two-dimensional random-cluster model is critical for $q \geq 1$. Probab. Theory Related Fields 153 511-542. MR2948685 
[5] Benjamini, I. (2010). Random planar metrics. In Proceedings of the International Congress of Mathematicians. Volume IV 2177-2187. Hindustan Book Agency, New Delhi. MR2827966

[6] BiskUP, M. (2017). Extrema of 2D discrete Gaussian free field. Lecture notes from the 2017 PIMS Summer School in Probability. Available at https://www.math.ucla.edu/ biskup/ PIMS/notes.html.

[7] Bouttier, J., Di Francesco, P. and Guitter, E. (2004). Planar maps as labeled mobiles. Electron. J. Combin. 11 Research Paper 69, 27. MR2097335

[8] Bramson, M., Ding, J. and Zeitouni, O. (2016). Convergence in law of the maximum of the two-dimensional discrete Gaussian free field. Comm. Pure Appl. Math. 69 62-123. MR3433630

[9] Cori, R. and VAuquelin, B. (1981). Planar maps are well labeled trees. Canad. J. Math. 33 1023-1042. MR0638363

[10] Ding, J. and Goswami, S. (2016). Upper bounds on Liouville first passage percolation and Watabiki's prediction. Preprint. Available at https://arxiv.org/abs/1610.09998.

[11] Ding, J. and Goswami, S. (2017). First passage percolation on the exponential of twodimensional branching random walk. Electron. Commun. Probab. 22 Paper No. 69, 14. MR3742400

[12] Ding, J. and ZHANG, F. (2017). Non-universality for first passage percolation on the exponential of log-correlated Gaussian fields. Probab. Theory Related Fields 171 1157-1188. MR3827229

[13] Duminil-Copin, H., Hongler, C. and Nolin, P. (2011). Connection probabilities and RSW-type bounds for the two-dimensional FK Ising model. Comm. Pure Appl. Math. 64 1165-1198. MR2839298

[14] Duminil-Copin, H., Manolescu, I. and Tassion, V. (2018). An RSW theorem for Gaussian free field. In preparation.

[15] Duminil-Copin, H., RaOufi, A. and TAssion, V. (2018). A new computation of the critical point for the planar random-cluster model with $q \geq 1$. Ann. Inst. Henri Poincaré Probab. Stat. 54 422-436. MR3765895

[16] Duminil-Copin, H., Sidoravicius, V. and Tassion, V. (2017). Continuity of the phase transition for planar random-cluster and Potts models with $1 \leq q \leq 4$. Comm. Math. Phys. 349 47-107. MR3592746

[17] Duplantier, B., Miller, J. and ShefField, S. (2014). Liouville quantum gravity as a mating of trees. Preprint. Available at http://arxiv.org/abs/1409.7055.

[18] Duplantier, B. and ShefField, S. (2011). Liouville quantum gravity and KPZ. Invent. Math. 185 333-393. MR2819163

[19] FerniQue, X. (1975). Regularité des trajectoires des fonctions aléatoires gaussiennes. In École d'Été de Probabilités de Saint-Flour, IV-1974. Lecture Notes in Math. 480 1-96. Springer, Berlin. MR0413238

[20] Grimmett, G. R. and Kesten, H. (2012). Percolation since Saint-Flour. In Percolation Theory at Saint-Flour. Probab. St.-Flour. Springer, Heidelberg. MR3014795

[21] Gwynne, E., Holden, N. and Sun, X. (2016). A distance exponent for Liouville quantum gravity. Preprint. Available at http://arxiv.org/abs/1606.01214.

[22] Lawler, G. F. and Limic, V. (2010). Random Walk: A Modern Introduction. Cambridge Studies in Advanced Mathematics 123. Cambridge Univ. Press, Cambridge. MR2677157

[23] LE GALL, J.-F. (2007). The topological structure of scaling limits of large planar maps. Invent. Math. 169 621-670. MR2336042

[24] Le Gall, J.-F. (2010). Geodesics in large planar maps and in the Brownian map. Acta Math. 205 287-360. MR2746349

[25] Le Gall, J.-F. (2013). Uniqueness and universality of the Brownian map. Ann. Probab. 41 2880-2960. MR3112934 
[26] Le Gall, J.-F. and Paulin, F. (2008). Scaling limits of bipartite planar maps are homeomorphic to the 2-sphere. Geom. Funct. Anal. 18 893-918. MR2438999

[27] Ledoux, M. (2001). The Concentration of Measure Phenomenon. Mathematical Surveys and Monographs 89. Amer. Math. Soc., Providence, RI. MR1849347

[28] Lyons, R. and Peres, Y. (2016). Probability on Trees and Networks. Cambridge Series in Statistical and Probabilistic Mathematics 42. Cambridge Univ. Press, New York. MR3616205

[29] Miermont, G. (2013). The Brownian map is the scaling limit of uniform random plane quadrangulations. Acta Math. 210 319-401. MR3070569

[30] Miermont, G. (2014). Aspects of random maps. Lecture Notes of the 2014 Saint-Flour Probability Summer School. Preliminary draft. Available at http://perso.ens-lyon.fr/gregory. miermont/coursSaint-Flour.pdf.

[31] Miller, J. and ShefField, S. (2015). Liouville quantum gravity and the Brownian map I: The QLE(8/3,0) metric. Preprint. Available at http://arxiv.org/abs/1507.00719.

[32] Miller, J. and SheFFIELD, S. (2016). Quantum Loewner evolution. Duke Math. J. $1653241-$ 3378. MR3572845

[33] Miller, J. and ShefField, S. (2016). Liouville quantum gravity and the Brownian map II: Geodesics and continuity of the embedding. Preprint. Available at http://arxiv.org/abs/ 1605.03563 .

[34] Miller, J. and Sheffield, S. (2016). Liouville quantum gravity and the Brownian map III: the conformal structure is determined. Preprint. Available at http://arxiv.org/abs/1608. 05391.

[35] PitT, L. D. (1982). Positively correlated normal variables are associated. Ann. Probab. 10 496-499. MR0665603

[36] Polyakov, A. M. (1981). Quantum geometry of bosonic strings. Phys. Lett. B 103 207-210. MR0623209

[37] Rhodes, R. and VArgas, V. (2014). Gaussian multiplicative chaos and applications: A review. Probab. Surv. 11 315-392. MR3274356

[38] Russo, L. (1978). A note on percolation. Z. Wahrsch. Verw. Gebiete 43 39-48. MR0488383

[39] Russo, L. (1981). On the critical percolation probabilities. Z. Wahrsch. Verw. Gebiete 56229 237. MR0618273

[40] SCHAEFFER, G. (1988). Conjugaison d'arbres et cartes combinatoires aléatoires. Ph.D. thesis. Univ. Bordeaux I.

[41] Seymour, P. D. and Welsh, D. J. A. (1978). Percolation probabilities on the square lattice. Ann. Discrete Math. 3 227-245. MR0494572

[42] Steele, J. M. (1986). An Efron-Stein inequality for nonsymmetric statistics. Ann. Statist. 14 753-758. MR0840528

[43] Tassion, V. (2016). Crossing probabilities for Voronoi percolation. Ann. Probab. $443385-$ 3398. MR3551200

DEPARTMENT OF STATISTICS

THE WHARTON SCHOOL

UNIVERSITY OF PENNSYLVANIA

3730 WALNUT STREET

Philadelphia, PENNSYlVANIA 19104

USA

E-MAIL: dingjian@wharton.upenn.edu
DEPARTMENT OF MATHEMATICS

STANFORD UNIVERSITY

450 SERra MaLl, BuILding 380

STANFORD, CALIFORNIA 94305

USA

E-MAIL: ajdun12@stanford.edu 